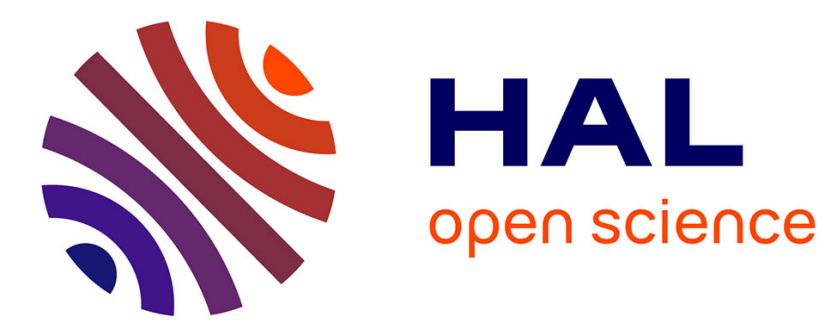

\title{
Elimination, fundamental principle and duality for analytic linear systems of partial differential-difference equations with constant coefficients
}

Henri Bourlès, Ulrich Oberst

\section{- To cite this version:}

Henri Bourlès, Ulrich Oberst. Elimination, fundamental principle and duality for analytic linear systems of partial differential-difference equations with constant coefficients. Mathematics of Control, Signals, and Systems, 2012, 10.1007/s00498-012-0089-8 . hal-02368155

\section{HAL Id: hal-02368155 \\ https://hal.science/hal-02368155}

Submitted on 20 Nov 2019

HAL is a multi-disciplinary open access archive for the deposit and dissemination of scientific research documents, whether they are published or not. The documents may come from teaching and research institutions in France or abroad, or from public or private research centers.
L'archive ouverte pluridisciplinaire HAL, est destinée au dépôt et à la diffusion de documents scientifiques de niveau recherche, publiés ou non, émanant des établissements d'enseignement et de recherche français ou étrangers, des laboratoires publics ou privés. 


\title{
Elimination, fundamental principle and duality for analytic linear systems of partial differential-difference equations with constant coefficients
}

published in Math. of Control, Signals and Systems 2012, DOI: 10.1007/s00498-012-0089-8

\author{
Henri Bourlès \\ SATIE, ENS Cachan/CNAM, \\ 61 Avenue President Wilson, F-94230 Cachan, France \\ email: henri.bourles@cnam.fr; tel:+33 140958883 \\ and \\ Ulrich Oberst \\ Institut für Mathematik, Universität Innsbruck \\ Technikerstraße 13, A-6020 Innsbruck, Austria \\ email: Ulrich.Oberst@uibk.ac.at, tel: +43 5125076073
}

July 26, 2012

\begin{abstract}
In this paper we investigate the solvability of inhomogeneous linear systems of partial differential-difference equations with constant coefficients and also the corresponding duality problem in how far the solutions of the corresponding homogeneous systems determine the equations. For ordinary delay-differential (DD) equations these behavioral problems were investigated in a seminal paper by GlüsingLürssen (1997) and in later papers by Habets, Glüsing-Lürssen, Vettori and Zampieri. In these papers the delay-differential operators are considered as distributions with compact support which act on smooth functions or on arbitrary distributions via convolution. The entire analytic Laplace transforms of the distributions with compact support play an important part in the quoted papers. In our approach the partial differential-difference operators belong to various topological operator rings $A$ of holomorphic functions on subsets of $\mathbb{C}^{n}$ and are thus studied in the frequency domain, the arguments of these (operator) functions being interpreted as generalized frequencies. We show that the topological duals $A^{\prime}$ of these operator rings with the canonical action of $A$ on $A^{\prime}$ have strong elimination and duality properties for ${ }_{A} A^{\prime}$-behaviors and admit concrete representations as spaces of analytic functions of systems theoretic interest. In particular, we study systems with generalized frequencies in the vicinity of suitable compact sets. An application to elimination for systems of periodic signals is given. We also solve an open problem of the quoted authors for DD-equations with incommensurate delays and analytic signals. Module theoretic methods in context with DD-equations have also been used by other authors, for instance by Fliess, Mounier, Rocha and Willems.
\end{abstract}


AMS-classification: 93B25, 93C05, 93C23, 93C 35

Keywords: partial differential-difference equation, fundamental principle, elimination, duality, multidimensional system, Stein algebra

\section{Contents}

1 Introduction

2 Duality and elimination in a general algebraic context

3 Analytic rings of operators and signal modules

4 Analytic local algebras

5 Holomorphic functions on compact Stein sets

6 The case of Stein algebras

7 Partial differential-difference equations

8 Characteristic variety and controllability

\section{Introduction}

In this paper we investigate the solvability of inhomogeneous linear systems of partial differential-difference equations with constant coefficients and also the corresponding duality problem in how far the solutions of the corresponding homogeneous systems determine the equations. For ordinary delay-differential (DD) equations these behavioral problems were investigated in the seminal paper [11] and then in [18], [13], [12], [30]. In these papers the delay-differential operators $T$ are considered as distributions with compact support which act on $\mathrm{C}^{\infty}$-functions or arbitrary distributions via convolution. The Laplace transforms $\widehat{T}(s)$ are entire and play an important part in the quoted papers. In our approach the partial differentialdifference operators belong to various topological operator rings $A$ of holomorphic functions on subsets of $\mathbb{C}^{n}$ and are thus studied in the frequency domain, the arguments of these functions being interpreted as generalized frequencies. We show that the topological duals $A^{\prime}$ of these operator rings with the canonical action of $A$ on $A^{\prime}$ furnish strong elimination and duality properties for ${ }_{A} A^{\prime}$-behaviors and admit concrete representations as spaces of analytic functions of systems theoretic interest. In particular, we study systems with generalized frequencies in the vicinity of suitable compact sets. An application to elimination for systems of periodic signals is given. We also solve an open problem of the quoted authors for DDequations with incommensurate delays and analytic signals. The present paper considerably extends, improves and simplifies work which was started three years ago and announced in [7]. Module theoretic methods in context with DD-equations have also been used by other authors, for instance by Fliess, Mounier [22], Rocha and Willems.

In a more general algebraic setting the properties of the title refer to generalized behaviors of signals whose components belong to a signal module ${ }_{A} W$ where $A$ is a not necessarily noetherian commutative ring of operators which acts on $W$ via $a \circ w$. Any submodule $U \subseteq A^{1 \times q}$ (rows) gives rise to its orthogonal generalized 
${ }_{A} W$-behavior

$$
\begin{gathered}
\mathcal{B}:=U^{\perp}:=\left\{w=\left(w_{1}, \cdots, w_{q}\right)^{\top} \in W^{q} \text { (columns); } U \circ w=0\right\}:= \\
\left\{w \in W^{q} ; \forall x=\left(x_{1}, \cdots, x_{q}\right) \in U: x \circ w=x_{1} \circ w_{1}+\cdots+x_{q} \circ w_{q}=0\right\} .
\end{gathered}
$$

If $U=A^{1 \times p} R, R \in A^{p \times q}$, is finitely generated (f.g.) by the rows of a matrix $R$

$$
\mathcal{B}:=U^{\perp}=\left\{w \in W^{q} ; R \circ w=0\right\}
$$

is just called an ${ }_{A} W$-behavior. If $A$ is noetherian behaviors and generalized behaviors coincide. Likewise any submodule $\mathcal{B} \subseteq W^{q}$ gives rise to its orthogonal module of equations $\mathcal{B}^{\perp}:=\left\{x \in A^{1 \times q} ; x \circ \mathcal{B}=0\right\}$. The maps $U \mapsto U^{\perp}$, $\mathcal{B} \mapsto \mathcal{B}^{\perp}$ form a Galois correspondence and induce a one-one correspondence between modules $U$ which satisfy $U=U^{\perp \perp}$ and generalized behaviors $\mathcal{B}=U^{\perp}$. A theorem which characterizes the modules $U$ with $U=U^{\perp \perp}$ is customarily called a duality theorem in behavioral systems theory. Shankar [27, Thm. 2.3] talks about the Nullstellensatz problem. If ${ }_{A} W$ is a cogenerator then all submodules $U$ satisfy $U=U^{\perp \perp}$ and are therefore in one-one correspondence with generalized behaviors.

If $P \in A^{q_{1} \times q_{2}}$ is a matrix and if $\mathcal{B}=U^{\perp} \subseteq W^{q_{2}}$ is a generalized behavior then the image $P \circ \mathcal{B}$ is not necessarily such again. If, however, images $P \circ \mathcal{B}$ of generalized behaviors are again such then we say that ${ }_{A} W$ admits elimination where we generalize Willems' terminology from standard one-dimensional continuous systems theory. In his original definition Willems considers the case

$\mathcal{B} \subseteq W^{q_{1}+n}, P=\left(\operatorname{id}_{q_{1}}, 0\right) \in A^{q_{1} \times\left(q_{1}+n\right)}, P \circ: W^{q_{1}+n} \rightarrow W^{q_{1}},(y, x)^{\top} \mapsto y$,

only and calls the components of $y$ resp. of $x$ the manifest resp. the latent variables of the behavior $\mathcal{B}$. If especially $P \circ W^{q_{2}}=(\operatorname{ker}(\circ P))^{\perp}$ with $\operatorname{ker}(\circ P)=$ $\left\{x \in A^{1 \times q_{1}} ; x P=0\right\}$ holds for all $P$ this signifies that an inhomogeneous linear system $P \circ y=u$ has a solution $y \in W^{q_{2}}$ if and only if the given right side $u \in W^{q_{1}}$ satisfies the necessary compatibility or integrability conditions $x \circ u=0$ for all $x$ with $x P=0$. Ehrenpreis introduced the terminology fundamental principle for this property of ${ }_{A} W$. The module $\operatorname{ker}(\circ P)$ is not f.g. in general and therefore the consideration of generalized behaviors is mandatory. These can be avoided if $A$ is coherent since then $\operatorname{ker}(\circ P)$ and other relevant modules are f.g. An injective module ${ }_{A} W$ admits elimination and satisfies the fundamental principle. The significance of injective cogenerator signal modules for multidimensional systems theory was first observed in [24]. Duality and elimination results are known and of highest importance in all approaches to linear systems theory and not only in the behavioral one. For the operator ring of complex multivariate polynomials which acts on various signal spaces $W$ of distributions the duality between modules and behaviors and the deviation of ${ }_{A} W$ from being injective or a cogenerator has been studied by Shankar in several papers, for instance in [26], [27].

Our considerations were inspired by the following simple standard example: If $k[s]=k\left[s_{1}, \cdots, s_{n}\right]$ denotes the $n$-variate polynomial algebra over any field $k$ its dual space

$$
\begin{gathered}
\operatorname{Hom}_{k}(k[s], k) \cong k[[x]]=k\left[\left[x_{1}, \cdots, x_{n}\right]\right] \cong k^{\mathbb{N}^{n}} \\
\varphi \leftrightarrow \sum_{\mu \in \mathbb{N}^{n}} \varphi\left(s^{\mu}\right) x^{\mu} \leftrightarrow w=\left(\varphi\left(s^{\mu}\right)\right)_{\mu \in \mathbb{N}^{n}}
\end{gathered}
$$

with the natural action is an injective cogenerator over $k[s]$. The signal space $k^{\mathbb{N}^{n}}$ is furnished with the natural action of $k[s]$ by left shifts and is one canonical signal module for discrete multidimensional systems theory. In the present paper we 
prove elimination and duality results for three types of locally convex topological algebras $A$ of convergent power series over $k=\mathbb{R}$ or $k=\mathbb{C}$ which act on their dual space $A^{\prime}$ of continuous linear functions $w: A \rightarrow k$ with the canonical action $(a \circ w)(b)=w(a b)$. In Section 3 we give concrete representations of the prototypical $A^{\prime}$ which explain their systems theoretic interest. We do not endow $A^{\prime}$ with a topology, but consider it as $A$-module only. For the systems theoretic applications of this paper such topologies are not needed, although they can be defined and may sometimes be of interest. The prototypical cases are the following:

1. Stein algebras [15]: The algebra $A$ is the $\mathbb{C}$-domain $\mathcal{O}\left(\mathbb{C}_{s}^{n}\right)$ of entire holomorphic functions in $n$ variables $s=\left(s_{1}, \cdots, s_{n}\right)$ with its Fréchet topology of compact convergence. This algebra is not noetherian, but is a Stein algebra [9], [15] with various essential topological properties. The elements of $A^{\prime}$ are called analytic functionals on $\mathbb{C}^{n}[20],[19, \S 4.5]$. The Laplace transform induces an $\mathcal{O}\left(\mathbb{C}_{s}^{n}\right)$-isomorphism

$$
\begin{gathered}
\mathcal{L}: \mathcal{O}\left(\mathbb{C}_{s}^{n}\right)^{\prime} \cong W:=\mathcal{O}\left(\mathbb{C}_{x}^{n} ; \exp \right), \varphi \mapsto \mathcal{L}(\varphi):=\varphi\left(e^{s \bullet x}\right), \text { with } \\
x=\left(x_{1}, \cdots, x_{n}\right) \in \mathbb{C}_{x}^{n}, s \bullet x=s_{1} x_{1}+\cdots+s_{n} x_{n}
\end{gathered}
$$

where $\mathcal{O}\left(\mathbb{C}_{x}^{n} ; \exp \right)$ is the space of entire holomorphic functions in $x \in \mathbb{C}^{n}$ of at most exponential growth and where the action $\circ$ of $\mathcal{O}\left(\mathbb{C}_{s}^{n}\right)$ on $W$ is the unique extension of that by partial differentiation, i.e.,

$$
s_{i} \circ w=\partial w / \partial x_{i} \text {, especially }\left(e^{y \bullet s} \circ w\right)(x)=w(x+y), x, y \in \mathbb{C}^{n} .
$$

Thus the ring $A$ contains differentiation and translation operators with which linear systems $R \circ w=0$ of partial differential-difference equations and their generalized solution behaviors can be defined. Note that in (4) we use the Laplace transform of analytic functionals for the construction of the interesting module $\mathcal{O}\left(\mathbb{C}_{x}^{n} ; \exp \right)$ of signals and not the Laplace transform of operators which are considered in the frequency domain from the beginning and whose arguments $s \in \mathbb{C}^{n}$ are therefore interpreted as generalized frequencies. Unless $\mathcal{O}\left(\mathbb{C}_{s}^{n}\right)$ is coherent which is not known, but unlikely for $n>1$ (communication of O. Forster) the consideration of generalized behaviors is mandatory. Th. 6.2 on duality and elimination for general Stein algebras is the main result in this context: The module ${ }_{A} W$ is an injective cogenerator for the category ${ }_{A} \mathbf{M o d}^{S t, f}$ of f.g. Stein modules [9], i.e., the functor $\operatorname{Hom}_{A}(-, W)$ is faithfully exact on this category. The submodules $U \subseteq A^{1 \times q}$ with $U=U^{\perp \perp}$ are exactly the closed ones whose factor modules $A^{1 \times q} / U$ are precisely the f.g. Stein modules, up to isomorphism. F.g. $U$ are closed and in oneone correspondence with the corresponding $A W$-behaviors $U^{\perp}$. One application of this result is Th. 6.5 on elimination for behaviors of periodic analytic signals defined by partial differential equations. The fundamental principle for such behaviors was recently studied in [23]. For any f.g. subgroup $G=\oplus_{j=1}^{m} \mathbb{Z} y^{(j)}$ of $\mathbb{C}^{n}$ we also consider the Laurent polynomial algebra of polynomial-exponential functions

$$
\begin{gathered}
\mathbb{C}\left[s, \sigma, \sigma^{-1}\right]=\oplus_{y \in G} \mathbb{C}[s] e^{y \bullet s} \subset A=\mathcal{O}\left(\mathbb{C}_{s}^{n}\right), \\
s=\left(s_{1}, \cdots, s_{n}\right), \sigma=\left(\sigma_{1}, \cdots, \sigma_{m}\right), \sigma_{j}:=e^{y^{(j)} \bullet s}, \sigma^{-1}=\left(\sigma_{1}^{-1}, \cdots, \sigma_{m}^{-1}\right),
\end{gathered}
$$

which is the least operator algebra containing the partial derivatives and the translations by $y^{(j)}$. For the case $n=m=1$ of ordinary delay-differential (DD) equations with commensurate delays and $\mathrm{C}^{\infty}$-signals Glüsing-Lürssen observed in the seminal paper [11] that for the validity of duality and elimination the noetherian ring $\mathbb{C}\left[s, \sigma, \sigma^{-1}\right]$ has to be replaced by its non-noetherian extension [3]

$$
B:=\operatorname{quot}\left(\mathbb{C}\left[s, \sigma, \sigma^{-1}\right]\right) \bigcap A=\mathbb{C}(s)\left[\sigma, \sigma^{-1}\right] \bigcap A \subset \operatorname{quot}(A)
$$


where quot denotes the quotient field and especially quot $(A)$ the field of meromorphic functions. The book [12] is an in-depth-study of the case $n=m=1$ for $\mathrm{C}^{\infty}$-signals. The case $n=1, m>1$ of DD-equations with incommensurate delays and $\mathrm{C}^{\infty}$-signals was discussed in [18] and [13]. In Theorems 7.1 and 7.3 we treat duality and elimination for the case $n \geq 1, m \geq 1$ and the signal module ${ }_{B} W$. Th. 7.7 seems to be the first elimination result for $n=1, m>1$ and solves an open problem of [13], but for analytic signals only. In Th. 8.3 we characterize controllable and spectrally controllable ${ }_{A} W$-behaviors.

2. Analytic local algebras [14]: The prototypical case is the noetherian $k$-domain $k<s>=k<s_{1}, \cdots, s_{n}>\subset k[[s]]$ of (locally) convergent power series over the real or complex fields field $k=\mathbb{R}, \mathbb{C}$. It is endowed with its locally convex sequence topology [14]. Any nonzero factor algebra $A=k\langle s\rangle / \mathfrak{a}$ with its coinduced locally convex topology is called an analytic local algebra [14]. In Th. 4.3 we show that for each such $A$ the dual space $A^{\prime}$ with its natural $A$-action is an injective cogenerator for the category ${ }_{A} \operatorname{Mod}$ of $A$-modules. This signifies that the functor $\operatorname{Hom}_{A}\left(-, A^{\prime}\right)$ is faithfully exact on the category of all $A$-modules and implies the strongest possible elimination and duality properties for the associated behaviors as was shown already in [24] for behaviors governed by pure partial differential equations. For $A=k\langle s\rangle$ the Laplace transform induces a $k<s>$-isomorphism of $k\left\langle s>^{\prime}\right.$ onto the space $\mathcal{O}\left(k_{x}^{n}\right.$; infexp) of everywhere convergent power series of infra-exponential, i.e. less than exponential, growth with the action from (5) [20] which contains all polynomials in particular. For the algebra $k\langle s-z\rangle$ of power series centered at $z \in k^{n}$ we obtain the injective cogenerator $W_{z}=\mathcal{O}\left(k_{x}^{n}\right.$; infexp) $e^{z \bullet x}$ with the action from (5). Since a power series $P \in k\langle s-z\rangle$ is locally convergent and defines a function for a generalized frequency $s$ near $z$ only we interpret a $k<s-z>W_{z}$-behavior as a system with generalized frequencies in the vicinity of $z$ only.

3. Noetherian rings of holomorphic functions on compact Stein subsets of a complex manifold: In Th. 5.6 we prove the injective cogenerator property of ${ }_{A} A^{\prime}$ where $A=\mathcal{O}(K)$ is the ring of holomorphic functions on a compact, connected, semi-analytic Stein subset $K$ of a complex manifold $Z$. Such an $A$ is noetherian. If $K$ is a compact semi-analytic and geometrically convex subset of $Z=\mathbb{C}^{n}$, for instance a compact polyhedron or polydisc, the Laplace transform induces an isomorphism $\mathcal{L}: \mathcal{O}(K)^{\prime} \cong W_{K}, \varphi \mapsto \varphi_{s}\left(e^{s \bullet x}\right)$, where $W_{K}$ is a subspace of $\mathcal{O}\left(\mathbb{C}_{x}^{n} ; \exp \right)$ of functions with explicitly described growth conditions (Th. 5.7). Again the interpretation of a $\mathcal{O}_{(K)} W_{K}$-behavior is that of a system with generalized frequencies in the vicinity of the compact set $K$.

Technique: The theorems of this paper and their proofs rely on deep results from the Theory of functions of several complex variables or Analytic Geometry, in particular on Stein spaces, manifolds, algebras and modules and on coherent analytic sheaves on Stein spaces [9], [19], [20], [16], [17], [29]. These results were already partially used in [24] and also by Shankar and Sule [28]. Other important ingredients of the proofs are fundamental results from Functional Analysis, in particular the Hahn/Banach theorem, the bipolar theorem, the open mapping theorem for Fréchet spaces and its generalizations to DFS-spaces (dual Fréchet-Schwartz spaces). The relevant f.g. $A$-modules $M$ are endowed with locally convex topologies for which one can show the exactness of the functor $M \mapsto M^{\prime}$ and the functorial isomorphism $M^{\prime} \cong \operatorname{Hom}_{A}\left(M, A^{\prime}\right)$ from which the elimination properties are inferred. Except in Section 5 no knowledge of analytic sheaves is required since we collect all needed algebraic and topological properties of the relevant algebras and modules in Results 4.2, 6.1 and 8.1 from the literature with exact quotations. In Section 5 we have to use the full machinery of several complex variables; we give precise references for all used results.

Acknowledgement: We thank O. Forster for various useful hints and explana- 
tions. We thank the referees for their efforts in reviewing this long and technically difficult paper.

\section{Duality and elimination in a general algebraic context}

We add some simple remarks on duality and elimination in a general algebraic context (compare [26]). Let ${ }_{A} W$ be a signal module as in the Introduction and let ${ }_{A}$ Mod denote the category of all $A$-modules with the $A$-linear maps. For all $p, q, r, \in \mathbb{N}$ there are the $A$-modules and $A$-bilinear forms

$$
\begin{gathered}
A^{p \times q}, W^{q \times r} \text { and } \circ: A^{p \times q} \times W^{q \times r} \rightarrow W^{p \times r},(f, w) \mapsto f \circ w, \\
f=\left(f_{i, j}\right)_{i, j} \in A^{p \times q}, w=\left(w_{j, k}\right)_{j, k} \in W^{q \times r}, \\
f \circ w:=\left((f \circ w)_{i, k}\right)_{i, k} \in W^{p \times r},(f \circ w)_{i, k}:=\sum_{j} f_{i, j} \circ w_{j, k}, \\
\text { especially } A^{1 \times q}=\{\text { rows }\}, W^{q}:=W^{q \times 1}=\{\text { columns }\}, \circ: A^{1 \times q} \times W^{q} \rightarrow W .
\end{gathered}
$$

For any $A$-module $M$ let $\mathbb{P}(M)$ denote the projective geometry of $M$, i.e., its ordered set of $A$-submodules. The bilinear form (8) induces the (order reversing) Galois correspondence (compare (1),(2))

$$
\begin{gathered}
\mathbb{P}\left(A^{1 \times q}\right) \leftrightarrows \mathbb{P}\left(W^{q}\right), U \rightarrow U^{\perp}, \mathcal{B}^{\perp} \leftarrow \mathcal{B}, \\
\text { with } U \subseteq U^{\perp \perp}, \mathcal{B} \subseteq \mathcal{B}^{\perp \perp}, U^{\perp}=U^{\perp \perp \perp}, \mathcal{B}^{\perp}=\mathcal{B}^{\perp \perp \perp} .
\end{gathered}
$$

This induces the order anti-isomorphism or duality

$$
\begin{array}{cccc}
\left\{U \in \mathbb{P}\left(A^{1 \times q}\right) ; U=U^{\perp \perp}\right\} & \cong & \left\{\mathcal{B} \in \mathbb{P}\left(W^{q}\right) ; \mathcal{B}=\mathcal{B}^{\perp \perp}\right\} \\
U=\mathcal{B}^{\perp} & \leftrightarrow & \mathcal{B}=U^{\perp}
\end{array}
$$

The orthogonal $U^{\perp}$ is also defined for all subsets $U \subseteq A^{1 \times q}$ and then $U^{\perp}=$ $\left({ }_{A}<U>\right)^{\perp}$ where ${ }_{A}<U>$ is the $A$-submodule generated by $U$. Likewise $\mathcal{B}^{\perp}$ is defined for all subsets of $W^{q}$. The submodule $U^{\perp}$ is called the generalized ${ }_{A} W$-behavior defined by $U$. The module $\mathcal{B}^{\perp}$ is called the module of equations of $\mathcal{B}$. The duality (10) obtains the form

$$
\left\{U \in \mathbb{P}\left(A^{1 \times q}\right) ; U=U^{\perp \perp}\right\} \cong\left\{\mathcal{B} \subseteq W^{q} ; W \text { is a generalized behavior }\right\} .
$$

Consider the standard $A$-basis $\delta_{i}=(0, \cdots, \stackrel{i}{1}, \cdots, 0) \in A^{1 \times q}, i=1, \cdots, q$. For $U \in \mathbb{P}\left(A^{1 \times q}\right)$ with generalized behavior $\mathcal{B}=U^{\perp}$ there is the obvious Malgrange isomorphism

$$
\operatorname{Hom}_{A}\left(A^{1 \times q} / U, W\right) \cong U^{\perp}=\mathcal{B}, \varphi \longleftrightarrow w, \varphi\left(\delta_{i}+U\right)=w_{i} .
$$

Since $\mathcal{B}=\mathcal{B}^{\perp \perp}$ the preceding isomorphism also induces the isomorphism

$$
\operatorname{Hom}_{A}\left(A^{1 \times q} / \mathcal{B}^{\perp}, W\right) \cong \mathcal{B}, \varphi \longleftrightarrow w, \varphi\left(\delta_{i}+\mathcal{B}^{\perp}\right)=w_{i} .
$$

The module $A^{1 \times q} / \mathcal{B}^{\perp}$ depends on the generalized behavior $\mathcal{B}$ only and induces the Malgrange isomorphism and is therefore called the module of $\mathcal{B}$. 
Lemma 2.1. Assume $P \in A^{q_{1} \times q_{2}}$, submodules $U_{i} \subseteq A^{1 \times q_{i}}$ with $U_{1} P \subseteq U_{2}$, and generalized behaviors $\mathcal{B}_{i} \underset{(12)}{\cong} \operatorname{Hom}_{A}\left(A^{1 \times q_{i}} / U_{i}, W\right)$. Then

$$
\begin{gathered}
\circ P: A^{1 \times q_{1}} \rightarrow A^{1 \times q_{2}}, x \mapsto x P, \text { induces the A-linear map } \\
\phi:=(\circ P)_{\text {ind }}: A^{1 \times q_{1}} / U_{1} \rightarrow A^{1 \times q_{2}} / U_{2}, \bar{x} \mapsto \overline{x P},
\end{gathered}
$$

and the commutative diagram

$$
\begin{array}{ccc}
\operatorname{Hom}_{A}\left(A^{1 \times q_{1}} / U_{1}, W\right) & \stackrel{\operatorname{Hom}(\phi, W)}{\longleftarrow} & \operatorname{Hom}_{A}\left(A^{1 \times q_{2}} / U_{2}, W\right) \\
\downarrow \cong(12) & & \downarrow \cong(12) \\
\mathcal{B}_{1}=U_{1}^{\perp} & \stackrel{P \circ}{\longleftarrow} & \mathcal{B}_{2}=U_{2}^{\perp}
\end{array} .
$$

This applies especially to any $U_{2}$ and $U_{1}:=(\circ P)^{-1}\left(U_{2}\right)$, the monomorphism

$$
\begin{gathered}
\phi=(\circ P)_{\text {ind }}: A^{1 \times q_{1}} /(\circ P)^{-1}\left(U_{2}\right) \rightarrow A^{1 \times q_{2}} / U_{2} \text { and } \\
P \circ: U_{2}^{\perp} \rightarrow\left((\circ P)^{-1}\left(U_{2}\right)\right)^{\perp} .
\end{gathered}
$$

Proof. The inclusion $U_{1} P \subseteq U_{2}$ implies that $\phi$ is well-defined. Let $\delta_{i}^{k}, i=$ $1, \cdots, q_{k}$, denote the standard bases of $A^{1 \times q_{k}}$ for $k=1,2$. Then

$$
\begin{gathered}
(\circ P)\left(\delta_{i}^{1}\right)=\delta_{i}^{1} P=P_{i-}=\sum_{j=1}^{q_{2}} P_{i j} \delta_{j}^{2}, \text { hence } \phi\left(\overline{\delta_{i}^{1}}\right)=\sum_{j=1}^{q_{2}} P_{i j} \overline{\delta_{j}^{2}} . \text { Let } \\
\varphi^{2} \in \operatorname{Hom}_{A}\left(A^{1 \times q_{2}} / U_{2}, W\right) \text { and } \varphi^{1}:=\operatorname{Hom}(\phi, W)\left(\varphi^{2}\right)=\varphi^{2} \phi .
\end{gathered}
$$

The Malgrange isomorphisms (12) map $\varphi^{k}$ onto $w^{k} \in W^{q_{k}}$ with $w_{i}^{k}:=\varphi^{k}\left(\overline{\delta_{i}^{k}}\right)$, hence

$$
\mathrm{w}_{i}^{1}=\varphi^{1}\left(\overline{\delta_{i}^{1}}\right)=\varphi^{2} \phi\left(\overline{\delta_{i}^{1}}\right)=\varphi^{2}\left(\sum_{j=1}^{q_{2}} P_{i j} \overline{\delta_{j}^{2}}\right)=\sum_{j=1}^{q_{2}} P_{i j} \circ \varphi^{2}\left(\overline{\delta_{j}^{2}}\right)=\sum_{j=1}^{q_{2}} P_{i j} \circ w_{j}^{2},
$$

hence $w^{1}=P \circ w^{2}$ and the commutativity of (15).

The following definition is a generalization of Willems' terminology.

Corollary and Definition 2.2. (Elimination, fundamental principle) Data of Lemma 2.1.

1. If $U_{2}=U_{2}^{\perp \perp}=\mathcal{B}_{2}^{\perp}$ then $P: U_{2}^{\perp} \rightarrow\left((\circ P)^{-1}\left(U_{2}\right)\right)^{\perp}$ and $\left((\circ P)^{-1}\left(U_{2}\right)\right)^{\perp}$ is the least generalized behavior containing $P \circ U_{2}^{\perp}$.

2. By definition elimination holds for generalized ${ }_{A} W$-behaviors if and only if the image of a generalized behavior is again such. By 1. this signifies that

$$
\left((\circ P)^{-1}\left(U_{2}\right)\right)^{\perp}=P \circ U_{2}^{\perp} \text { for all } P \text { and } U_{2}=U_{2}^{\perp \perp} .
$$

3. If $P \circ W^{q_{2}}=\operatorname{ker}(\circ P)^{\perp}$ holds for all $P$ as before and $U_{2}:=0$, hence $U_{2}^{\perp}=W^{q}$ and $(\circ P)^{-1}\left(U_{2}\right)=\operatorname{ker}(\circ P)$ then ${ }_{A} W$ is said to satisfy the fundamental principle. If, in addition, $\operatorname{ker}(\circ P)=A^{1 \times k} Q$ is finitely generated by the rows of a matrix $Q \in A^{k \times q_{1}}$ then the inhomogeneous linear system $P \circ y=u$ has a solution $y$ if and only if $Q \circ u=0$. If ${ }_{A} W$ is a faithful module, i.e., $W^{\perp}=0$ and thus $0=0^{\perp \perp}=\left(W^{q_{2}}\right)^{\perp}$ for all $q_{2}$ and $U_{2}:=0 \subseteq A^{1 \times q_{2}}$, then elimination implies the fundamental principle.

4. If ${ }_{A} W$ is an injective module then the monomorphism $\phi$ in (16) gives rise to the epimorphisms $\operatorname{Hom}(\phi, W)$ and $P \circ: U_{2}^{\perp} \rightarrow\left((\circ P)^{-1}\left(U_{2}\right)\right)^{\perp}$ in (15) for all submodules $U_{2} \subseteq A^{1 \times q_{2}}$, and especially elimination holds for generalized ${ }_{A} W$-behaviors. 
The last statement of item 1 . of the preceding corollary is easily checked. Notice that its item 2. does not imply that the image of a $W$-behavior is again such because it may occur in (16) that $U_{2}$ is finitely generated, but $(\circ P)^{-1}\left(U_{2}\right)$ is not since $A$ is not assumed noetherian.

Definition and Corollary 2.3. ( Coherence [4, §I.2, Ex. 11,12 on p.44]) By definition an $A$-module is finitely generated (f.g.) resp. finitely presented (f.p.) if it is isomorphic to a module of the form $A^{1 \times q} / U$ with any resp. a f.g. submodule $U$. Let ${ }_{A} \operatorname{Mod}^{f}\left({ }_{A} \operatorname{Mod}^{f p}\right)$ denote the full subcategories of ${ }_{A} \operatorname{Mod}$ of all f.g. (f.p.) modules. An $A$-module $M$ is called coherent if it is f.g. and if each f.g. submodule is f.p.. This definition applies especially to $A$ considered as $A$-module. A coherent module is f.p.. In an exact sequence $0 \rightarrow M_{1} \rightarrow M_{2} \rightarrow M_{3} \rightarrow 0$ all three $M_{i}$ are coherent if and only two of them have this property. This implies that homomorphisms between coherent modules have coherent kernel, image and cokernel. The ring $A$ is coherent if and only if the annihilator $\operatorname{ann}_{A}(a):=\{f \in A ; f a=0\}$ of each element $a \in A$ and the intersection of any two f.g. ideals are again f.g. If $A$ is an integral domain the annihilator condition is trivially satisfied. If $A$ is coherent then the coherent modules are precisely the finitely presented ones, i.e., form the category ${ }_{A} \operatorname{Mod}^{f p}$. If $A$ is coherent and if $U_{2}$ in (16) is f.g. the kernel

$$
(\circ P)^{-1}\left(U_{2}\right)=\operatorname{ker}\left((\circ P)_{\text {ind }}: A^{1 \times q_{1}} \rightarrow A^{1 \times q_{2}} / U_{2}\right)
$$

is coherent and thus f.g. This implies that $\left((\circ P)^{-1}\left(U_{2}\right)\right)^{\perp}$ is a behavior.

Lemma 2.4. Assume that $A$ is coherent and that $W$ satisfies the following weak Baer condition: For any f.g. ideal $\mathfrak{a}$ of $A$ the map $W \rightarrow \operatorname{Hom}_{A}(\mathfrak{a}, W), w \mapsto$ $(f \mapsto f \circ w)$, is surjective. If in (16)

$$
U_{2} \text { is f.g. then so is }(\circ P)^{-1}\left(U_{2}\right) \text { and } P \circ U_{2}^{\perp}=\left((\circ P)^{-1}\left(U_{2}\right)\right)^{\perp} \text {. }
$$

In particular, the image of a behavior is a behavior.

Proof. Equation (17) implies that the map $\phi$ from (16) is a monomorphism between f.g. modules. It suffices to show that $\operatorname{Hom}(\phi, W)$ is surjective. Let, more generally, $U$ be any f.g. submodule of a f.g. module $M$ and let inj : $U \rightarrow M$ be the canonical injection. We show that the restriction

$$
\operatorname{Hom}_{A}(M, W) \rightarrow \operatorname{Hom}_{A}(U, W), \varphi \mapsto \varphi \mid U=\varphi \operatorname{inj},
$$

is surjective. Since $M$ admits a filtration $U_{0} \subseteq U_{1} \subseteq \cdots \subseteq U_{k}=M$ with f.g. $U_{i}$ and cyclic factors $U_{i} / U_{i-1}$ it suffices, via induction, to prove the surjectivity of (18) for the case that $M / U$ is cyclic. So let $M / U=A \bar{x}$ or $M=U+A x$ and consider the annihilator ideal

$$
\mathfrak{a}:=\operatorname{ker}(A \rightarrow M / U, f \mapsto f(x+U))=\{f \in A ; f x \in U\} .
$$

It is f.g. since $A$ and $M / U$ are coherent. Let $\psi: U \rightarrow M$ be $A$-linear. By the weak Baer condition there is a $w \in W$ such that

$$
\mathfrak{a} \stackrel{\cdot x}{\longrightarrow} U \stackrel{\psi}{\longrightarrow} W, f \mapsto \psi(f x), \text { has the form } \psi(f x)=f \circ w, f \in \mathfrak{a} .
$$

Define the map

$$
\varphi: M=U+A x \rightarrow W, u+f x \mapsto \psi(u)+f \circ w .
$$

This is well-defined since

$$
\begin{gathered}
u_{1}+f_{1} x=u_{2}+f_{2} x \Longrightarrow\left(f_{2}-f_{1}\right) x=u_{1}-u_{2} \in U \Longrightarrow f_{2}-f_{1} \in \mathfrak{a} \Longrightarrow \\
\psi\left(u_{1}-u_{2}\right)=\psi\left(\left(f_{2}-f_{1}\right) x\right)=\left(f_{2}-f_{1}\right) \circ w \Longrightarrow \psi\left(u_{1}\right)+f_{1} \circ w=\psi\left(u_{2}\right)+f_{2} \circ w .
\end{gathered}
$$

Obviously $\varphi$ is an $A$-linear extension of $\psi$, i.e., $\varphi \mid U=\psi$. 
The following lemma is well-known.

Lemma and Definition 2.5. (Willems closure, cogenerator, compare [26, §2]) If $U \in \mathbb{P}\left(A^{1 \times q}\right), M:=A^{1 \times q} / U$ and $\mathcal{B}:=U^{\perp}$ then $U^{\perp \perp} / U=\mathcal{B}^{\perp} / U$ is the kernel of the linear map

$$
\begin{array}{ccccc}
\operatorname{can}_{M}: \quad M=A^{1 \times q} / U & \rightarrow & W^{\mathcal{B}} & \cong & W^{\operatorname{Hom}_{A}(M, W)} \\
\bar{x}=x+U & \mapsto \quad(x \circ w)_{w \in \mathcal{B}} & \mapsto & (\varphi(\bar{x}))_{\varphi \in \operatorname{Hom}_{A}(M, W)} .
\end{array}
$$

The module $U^{\perp \perp}$ is also called the Willems closure of $U$ with respect to (w.r.t.) $W$. If $U^{\perp \perp}=U$ then $U$ is called Willems closed w.r.t. $W$. The duality (11) thus establishes a duality between Willems closed modules and generalized behaviors.

The module $W$ is called a cogenerator for f.g. modules if for every f.g. Amodule $M=A^{1 \times q} / U$ the map $\operatorname{can}_{M}$ is injective or, equivalently, $U=U^{\perp \perp}$ or, in still other words, if $\operatorname{Hom}_{A}(M, W)$ separates the elements of $M$, hence especially $\operatorname{Hom}_{A}(M, W) \neq 0$ if $M \neq 0$. Then (11) establishes a duality between $\mathbb{P}\left(A^{1 \times q}\right)$ and the set of generalized behaviors in $W^{q}$. The module ${ }_{A} W$ is called $a$ cogenerator if $\operatorname{can}_{M}: M \rightarrow W^{\mathrm{Hom}_{A}(M, W)}$ is injective for all $A$-modules $M$.

\section{Analytic rings of operators and signal mod- ules}

We introduce the rings of operators and signal spaces relevant for this paper.

Let $k$ denote the field of real numbers $\mathbb{R}$ or of complex numbers $\mathbb{C}$. Let $n \in \mathbb{N}$ and

$k[[s]]:=k\left[\left[s_{1}, \cdots, s_{n}\right]\right]=\left\{f=\sum_{\mu \in \mathbb{N}^{n}} f_{\mu} s^{\mu}, f_{\mu} \in k\right\}, s^{\mu}:=s_{1}^{\mu_{1}} s_{2}^{\mu_{2}} \cdots s_{n}^{\mu_{n}}$

be the $k$-algebra of formal power series in indeterminates $s_{1}, \cdots, s_{n}$ and likewise $k[[x]]:=k\left[\left[x_{1}, \cdots, x_{n}\right]\right]$. Following [14] and [19] we introduce the algebras of locally convergent and of entire or everywhere convergent power series. For this purpose we consider the ordered set $\mathbb{R}_{+}^{n}$ of real vectors $T=\left(T_{1}, \cdots, T_{n}\right)$ with positive entries $T_{i}>0$. With the componentwise order this set is directed upwards and downwards. We write $T \leq S$ resp. $T<S$ if $T_{i} \leq S_{i}$ resp. $T_{i}<S_{i}$ for all $i$. For $T \in \mathbb{R}_{+}^{n}$ we introduce the poly-cylinder [14]

$$
\begin{gathered}
Z(T):=\left\{z \in k^{n} ; \forall i=1, \cdots, n:\left|z_{i}\right|<T_{i}\right\} \subset \\
\overline{Z(T)}:=\left\{z \in k^{n} ; \forall i=1, \cdots, n:\left|z_{i}\right| \leq T_{i}\right\}, \\
|f|_{T}:=\sum_{\mu}\left|f_{\mu}\right| T^{\mu} \leq \infty, B_{T}:=\left\{f \in k[[s]] ;|f|_{T}<\infty\right\}, \\
\forall T \leq S, f \in k[[s]]:|f|_{T} \leq|f|_{S}, \text { hence } B_{S} \subseteq B_{T} .
\end{gathered}
$$

The algebra $B_{T}$ is a $k$-Banach subalgebra of $k[[s]]$ with the norm $|-|_{T}[14$, Satz 1 on p.16]. Consider the algebra $C^{0}(\overline{Z(T)})$ of continuous $k$-valued functions on the compact set $\overline{Z(T)}$ with the maximum norm $\|f\|_{T}:=\max _{z \in \overline{Z(T)}}|f(z)|$. Every $f \in B_{T}$ defines the function

$$
f: \overline{Z(T)} \rightarrow k, z \mapsto f(z):=\sum_{\mu \in \mathbb{N}^{n}} f_{\mu} z^{\mu}, \text { with }
$$

$f \in C^{0}(\overline{Z(T)})$ and $\|f\|_{T} \leq|f|_{T}$, hence inj : $B_{T} \subseteq C^{0}(\overline{Z(T)})$ 
is a contraction and thus continuous. For the complex case $k=\mathbb{C}$ Cauchy's inequalities [19, Th. 2.2.7] imply

$$
\left|f_{\mu}\right|=\frac{\left|f^{(\mu)}(0)\right|}{\mu !} \leq\|f\|_{T} T^{-\mu} .
$$

The algebras of entire or everywhere convergent resp. of locally convergent power series are then defined as

$$
\begin{gathered}
\mathcal{O}\left(k_{s}^{n}\right):=\bigcap_{T \in \mathbb{R}_{+}^{n}} B_{T} \subset k<s>:=\bigcup_{T \in \mathbb{R}_{+}^{n}} B_{T}= \\
\left\{f \in k[[s]] ; \exists z \in k^{n} \text { with } z_{i} \neq 0, i=1, \cdots, n \text {, and convergent } \sum_{\mu \in \mathbb{N}^{n}} f_{\mu} z^{\mu} .\right\}
\end{gathered}
$$

Both carry a natural topology. The algebra $\mathcal{O}\left(k_{s}^{n}\right)$ carries the initial or induced topology with respect to all inclusions $\mathcal{O}\left(k_{s}^{n}\right) \subset B_{T}, T \in \mathbb{R}_{+}^{n}$. By this it is a Fréchet algebra and a sequence in $\mathcal{O}\left(k_{s}^{n}\right)$ converges if and only if it converges in all $B_{T}$.

Remark 3.1. The inequality $\|-\|_{T} \leq|-|_{T}$ implies that the natural topology on $\mathcal{O}\left(k_{s}^{n}\right)$ is finer than the topology of compact convergence for $k=\mathbb{R}, \mathbb{C}$. In the complex case $\mathcal{O}\left(\mathbb{C}_{s}^{n}\right)$ is the algebra of holomorphic functions in variables $s_{1}, \cdots, s_{n}$ on $\mathbb{C}^{n}$. For $f \in \mathcal{O}\left(\mathbb{C}^{n}\right)$ and $T<S$ the inequality (22) implies

$$
\begin{gathered}
|f|_{T}=\sum_{\mu}\left|f_{\mu}\right| T^{\mu}=\sum_{\mu}\left|f_{\mu}\right| S^{\mu}\left(\frac{T}{S}\right)^{\mu} \leq \\
\left(\sum_{\mu}\left(\frac{T}{S}\right)^{\mu}\right)\|f\|_{S}=C\|f\|_{S} \text { with } C:=\sum_{\mu}\left(\frac{T}{S}\right)^{\mu}
\end{gathered}
$$

and thus that the natural topology coincides with the topology of compact convergence. The natural topology of $\mathcal{O}\left(\mathbb{R}_{s}^{n}\right)$ is finer, but not equal to that of compact convergence. Notice that Cauchy's inequality (11) is not valid in the real case.

The natural topology on $k<s>$ is the sequence topology discussed in [14, §I.3.4, §I.6, §I.7]. It is the final topology induced by all injections $B_{T} \subseteq k<$ $s>$. If $f_{k}, k \in \mathbb{N}$, and $f$ belong to $k<s>$ then the $f_{k}$ converge to $f$ in the sequence topology if and only if there is a $T \in \mathbb{R}_{+}^{n}$ such that $f_{k}, f \in B_{T}$ and $f=\lim _{k} f_{k}$ in $B_{T}$ [14, Satz I.7.8 on p.67]. A map from $k<s>$ to a topological space is continuous if and only if it preserves convergent sequences. The injection $\mathcal{O}\left(k_{s}^{n}\right) \subset k<s>$ is obviously continuous. The ring $k\langle s\rangle$ is noetherian [14, Satz I.5.3 on p.45] and local with the unique maximal ideal [14, I.3.1 on p.27]

$$
\mathfrak{m}:=\left\{f \in k<s>; f(0)=f_{0}=0\right\}=\sum_{i=1}^{n} k<s>s_{i}
$$

and the residue field $k\langle s\rangle / \mathfrak{m}=k$. With the sequence topology $k\langle s>$ is a Hausdorff topological algebra [14, Satz I.6.3 on p.58 and Satz I.7.7 on p.66], locally convex [14, Satz I.8.7 on p.74], sequence-complete [14, Satz I.8.6 on p.74], i.e., every Cauchy sequence converges, but does not have countable neighborhood bases [14, Satz I.7.9 on p.67] and is therefore not metrizable. We use the same topologies on $k\langle x\rangle$ and $\mathcal{O}\left(k_{x}^{n}\right)$. Below we will discuss and essentially use the category of f.g. $k<s>$-modules with their sequence topology [14, Kap.II, §0, $\S 1]$.

We now introduce the signal spaces of the present paper. Let $k<s>^{\prime}$ and 
$\mathcal{O}\left(k_{s}^{n}\right)^{\prime}$ denote the topological dual spaces, i.e., the $k$-spaces of continuous $k$ linear functions on $k<s>$ resp. $\mathcal{O}\left(k_{s}^{n}\right)$ into the topological field $k$. With the help of [19] it was shown in [24, Th.4.20 on p.67] that the $k$-bilinear map

$$
\langle-,-\rangle: k<s>\times \mathcal{O}\left(k_{x}^{n}\right) \rightarrow k,\langle f, w\rangle=\left\langle\sum_{\mu} f_{\mu} s^{\mu}, \sum_{\mu} w_{\mu} x^{\mu}\right\rangle:=\sum_{\mu} f_{\mu} w_{\mu},
$$

is well-defined and non-degenerate in the strong sense that it induces isomorphisms

$$
\begin{gathered}
k<s>^{\prime} \cong \mathcal{O}\left(k_{x}^{n}\right), \varphi=\langle-, w\rangle \leftrightarrow w=\sum_{\mu} \varphi\left(s^{\mu}\right) x^{\mu} \\
k<s>\cong \mathcal{O}\left(k_{x}^{n}\right)^{\prime}, f=\sum_{\mu} \psi\left(x^{\mu}\right) s^{\mu} \leftrightarrow \psi=\langle f,-\rangle, \text { hence also }(s \leftrightarrow x) \\
\mathcal{O}\left(k_{s}^{n}\right)^{\prime} \cong k<x>, \varphi=\langle-, w\rangle \leftrightarrow w=\sum_{\mu} \varphi\left(s^{\mu}\right) x^{\mu} .
\end{gathered}
$$

The dual spaces are not considered as topological here. The proof in [24, Th.4.20 on p.67] was given for $k=\mathbb{C}$, but the proof for $k=\mathbb{R}$ is the same. Recall that the topology on $\mathcal{O}\left(\mathbb{R}_{s}^{n}\right)$ is finer than the topology of compact convergence. Since the algebras $\mathcal{O}\left(k_{s}^{n}\right)$ and $k<s>$ are topological their dual spaces are modules over them with the action or scalar multiplication

$$
(f \circ \varphi)(g):=\varphi(f g) \text { for }\left\{\begin{array}{l}
f, g \in \mathcal{O}\left(k_{s}^{n}\right), \varphi \in \mathcal{O}\left(k_{s}^{n}\right)^{\prime} \\
f, g \in k<s>, \varphi \in k<s>^{\prime}
\end{array} .\right.
$$

Via the isomorphisms from (27) we obtain modules ${\mathcal{O}\left(k_{s}^{n}\right)} k<x>$ and $_{k<s>} \mathcal{O}\left(k_{x}^{n}\right)$ with the actions

$$
\begin{aligned}
f \circ w,\langle g, f \circ w\rangle= & \langle f g, w\rangle \text { for }\left\{\begin{array}{l}
f, g \in \mathcal{O}\left(k_{s}^{n}\right), w \in k<x>, \\
f, g \in k<s>, w \in \mathcal{O}\left(k_{x}^{n}\right),
\end{array} \quad\right. \text { with } \\
& s^{\nu} \circ \sum_{\mu} w_{\mu} x^{\mu}=\sum_{\mu} w_{\nu+\mu} x^{\mu} .
\end{aligned}
$$

Therefore these actions are extensions of the left shift action of the polynomial algebra $k[s]$ on the multi-sequence space $k^{\mathbb{N}^{n}}=k[[x]]$ to the larger rings of operators and smaller signal spaces $\mathcal{O}\left(k_{x}^{n}\right) \subset k\langle x>\subset k[[x]]$. Therefore these signal modules are suitable for partial difference equations and discrete systems theory. The canonical isomorphism

$$
\text { can }: k[[x]] \cong k[[x]], \sum_{\mu} w_{\mu} x^{\mu} \mapsto \sum_{\mu} \frac{w_{\mu}}{\mu !} x^{\mu},
$$

induces isomorphisms on the spaces of convergent power series. For this purpose we introduce growth conditions for entire holomorphic functions, first in the complex case. Let $\|-\|$ denote any norm on $\mathbb{C}^{n}$ and then define

$$
\begin{gathered}
\mathcal{O}\left(\mathbb{C}_{x}^{n} ; \exp \right):=\left\{w \in \mathcal{O}\left(\mathbb{C}_{x}^{n}\right) ; \exists \delta>0 \exists C_{\delta}>0 \forall x \in \mathbb{C}^{n}:|w(x)| \leq C_{\delta} e^{\delta|| x||}\right\} \supset \\
W_{0}:=\mathcal{O}\left(\mathbb{C}_{x}^{n} ; \operatorname{infexp}\right):= \\
\left\{w \in \mathcal{O}\left(\mathbb{C}_{x}^{n}\right) ; \forall \delta>0 \exists C_{\delta}>0 \forall x \in \mathbb{C}^{n}:|w(x)| \leq C_{\delta} e^{\delta|| x||}\right\} .
\end{gathered}
$$

The functions in $\mathcal{O}\left(\mathbb{C}_{x}^{n} ; \exp \right)$ resp. in $\mathcal{O}\left(\mathbb{C}_{x}^{n} ;\right.$ infexp $)$ are called of at most exponential resp. of infra-exponential growth. Since all norms on $\mathbb{C}^{n}$ are equivalent 
the definition does not depend on the choice of $\|-\|$ on $\mathbb{C}^{n}$. With the help of [19] it was shown in [24, Th. 4.27] that can from (30) induces the $\mathbb{C}$-isomorphism

$$
\text { can : } \mathbb{C}<x>\cong \mathcal{O}\left(\mathbb{C}_{x}^{n} ; \exp \right) .
$$

The same proof furnishes the $\mathbb{C}$-isomorphism

$$
\operatorname{can}: \mathcal{O}\left(\mathbb{C}_{x}^{n}\right) \cong \mathcal{O}\left(\mathbb{C}_{x}^{n} ; \text { infexp }\right) .
$$

In the real case we define

$$
\begin{gathered}
\mathcal{O}\left(\mathbb{R}_{x}^{n} ; \exp \right):=\mathcal{O}\left(\mathbb{R}_{x}^{n}\right) \bigcap \mathcal{O}\left(\mathbb{C}_{x}^{n} ; \exp \right) \supset \\
\mathcal{O}\left(\mathbb{R}_{x}^{n} ; \text { infexp }\right):=\mathcal{O}\left(\mathbb{R}_{x}^{n}\right) \bigcap \mathcal{O}\left(\mathbb{C}_{x}^{n} ; \text { infexp }\right) .
\end{gathered}
$$

Obviously the isomorphisms (32) and (33) then also hold with $\mathbb{C}$ replaced by $\mathbb{R}$. By transport of structure we replace the signal spaces in equations (26) to (29) by the isomorphic ones from (32) to (34) and obtain the following

Corollary and Definition 3.2. Let $k=\mathbb{R}, \mathbb{C}$. The $k$-bilinear forms

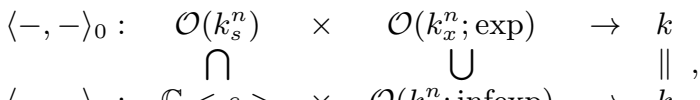

$$
\begin{aligned}
& \left\langle\sum_{\mu} f_{\mu} s^{\mu}, \sum_{\mu} w_{\mu} x^{\mu}\right\rangle_{0}:=\sum_{\mu} f_{\mu} w_{\mu} \mu !,
\end{aligned}
$$

are well-defined and non-degenerate and induce the Laplace transform isomorphisms

$$
\begin{array}{cccc}
\mathcal{L}: & \mathcal{O}\left(k_{s}^{n}\right)^{\prime} & \cong & \mathcal{O}\left(k_{x}^{n} ; \exp \right) \\
& \bigcup & & \mathcal{O}\left(k_{x}^{n} ; \text { infexp }\right) \\
\mathcal{L}: & k<s>^{\prime} & \cong & \\
& \varphi=\langle-, w\rangle_{0} & \leftrightarrow & w=\mathcal{L}(\varphi)=\sum_{\mu} \frac{\varphi\left(s^{\mu}\right)}{\mu !} x^{\mu}=\varphi\left(e^{s \bullet x}\right)
\end{array}
$$

where $s \bullet x:=s_{1} x_{1}+\cdots+s_{n} x_{n}$. These are $\mathcal{O}\left(k_{s}^{n}\right)$ - resp. $k<s>$-isomorphisms if the power series spaces in $x$ are equipped with the action $\circ_{0}$ defined by

$$
\begin{gathered}
\left\langle g, f \circ_{0} w\right\rangle_{0}=\langle f g, w\rangle_{0} . \text { Then } \\
s^{\mu} \circ_{0} w=\partial^{\mu} w \text { and }\left(e^{z \bullet s} \circ_{0} w\right)(x)=w(x+z), x, z \in k^{n},
\end{gathered}
$$

where $\partial:=\left(\partial / \partial x_{1}, \cdots, \partial / \partial x_{n}\right)$. The isomorphisms (32) and (33) are $\mathcal{O}\left(k_{s}^{n}\right)$ resp. $k<s>$-linear and thus establish an isomorphism between discrete and continuous analytic signal modules and, of course, $\mathcal{O}\left(k_{x}^{n} ; \operatorname{infexp}\right)$ is an $\mathcal{O}\left(k_{s}^{n}\right)$ submodule of $\mathcal{O}\left(k_{x}^{n} ; \exp \right)$.

The formula $\left(e^{z \bullet s} \circ_{0} w\right)(x)=w(x+z)$ implies that the action of $\mathcal{O}\left(k_{s}^{n}\right)$ on analytic signals realizes the translation action in particular. Therefore we later consider the subring

$$
\oplus_{z \in k^{n}} k[s] e^{z \bullet s} \subset \mathcal{O}\left(k_{s}^{n}\right)
$$

of polynomial-exponential functions which acts on $\mathcal{O}\left(k_{x}^{n} ; \exp \right)$ by partial differentialdifference operators, the term difference referring to the translation action.

We finally extend the preceding considerations from the center 0 to arbitrary points of $k^{n}$. Let $z \in k^{n}$ be such a point and $k<s-z>$ the ring of locally convergent power series at $z$ with the sequence topology which is defined like that of $k\langle s\rangle$. Since $k[s]=k[s-z] \subset \mathcal{O}\left(k_{s}^{n}\right) \subset k\langle s-z>$ and since $k[s-z]$ is dense in $k<s-z>$ so is $\mathcal{O}\left(k_{s}^{n}\right)$ and therefore we identify

$$
\begin{gathered}
k<s-z>^{\prime} \subset \mathcal{O}\left(k_{s}^{n}\right)^{\prime}, \varphi=\varphi \mid \mathcal{O}\left(k_{s}^{n}\right) \text { for } \varphi \in k<s-z>^{\prime}, \text { and define } \\
W_{z}:=\mathcal{L}\left(k<s-z>^{\prime}\right) \subset \mathcal{O}\left(k_{x}^{n}\right)
\end{gathered}
$$


where, according to Cor. 3.2, $\mathcal{L}: \mathcal{O}\left(k_{s}^{n}\right)^{\prime} \cong \mathcal{O}\left(k_{x}^{n} ; \exp \right)$ is the Laplace transform and $W_{0}=\mathcal{O}\left(k_{x}^{n} ;\right.$ infexp $)$. Moreover there are the topological translation isomorphisms

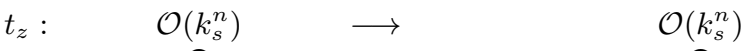

$$
\begin{aligned}
& t_{z}: \quad \bigcap \quad \longrightarrow \quad \longrightarrow \quad k<s-z> \\
& f=\sum_{\mu \in \mathbb{N}^{n}} f_{\mu} s^{\mu} \quad \mapsto \quad t_{z}(f):=f(s-z)=\sum_{\mu \in \mathbb{N}^{n}} f_{\mu}(s-z)^{\mu} \\
& \text { with their adjoints }
\end{aligned}
$$

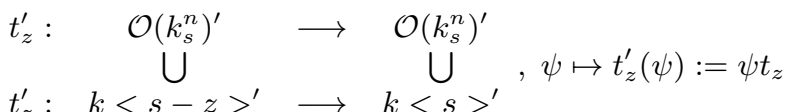

Obviously $t_{-z}$ is the inverse of $t_{z}$.

Lemma 3.3. The following diagrams of $k$-isomorphisms commute:

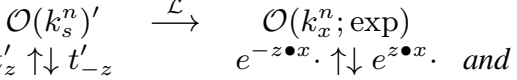

$$
\begin{aligned}
& \mathcal{O}\left(k_{s}^{n}\right)^{\prime} \stackrel{\mathcal{L}}{\longrightarrow} \quad \mathcal{O}\left(k_{x}^{n} ; \exp \right) \\
& k<s>^{\prime} \quad \stackrel{\mathcal{L}}{\longrightarrow} \quad W_{0}=\mathcal{O}\left(k_{x}^{n} ; \text { infexp }\right) \\
& t_{z}^{\prime} \uparrow \downarrow t_{-z}^{\prime} \quad e^{-z \bullet x} \cdot \uparrow \downarrow e^{z \bullet x} \text {, hence } W_{z}=e^{z \bullet x} W_{0} \text {. } \\
& k<s-z>^{\prime} \stackrel{\mathcal{L}}{\longrightarrow} W_{z}=\mathcal{L}\left(k<s-z>^{\prime}\right)
\end{aligned}
$$

Proof. For $\varphi \in \mathcal{O}\left(k_{s}^{n}\right)^{\prime}$ we indeed have

$$
\begin{gathered}
\mathcal{L} t_{z}^{\prime}(\varphi)=\mathcal{L}\left(\varphi t_{z}\right)=\varphi\left(t_{z}\left(e^{s \bullet x}\right)\right)= \\
\varphi\left(e^{(s-z) \bullet x}\right)=\varphi\left(e^{-z \bullet x} e^{s \bullet x}\right)=e^{-z \bullet x} \mathcal{L}(\varphi) .
\end{gathered}
$$

Assume that

$w \in \mathcal{O}\left(k_{x}^{n} ; \exp \right)$ with $|w(x)| \leq C_{\delta} e^{\delta|| x||}$ for some $\delta, C_{\delta}>0$ and all $x \in \mathbb{C}^{n}$ where $\|x\|$ is a norm on $\mathbb{C}^{n}$. Since $\|x-z\| \leq\|x\|+\|z\|$ we infer

$$
\begin{aligned}
&\left|t_{z}(w)(x)\right|=|w(x-z)| \leq C_{\delta} e^{\delta|| z||} e^{\delta|| x||} \text { and } t_{z}(w) \in \mathcal{O}\left(k_{x}^{n} ; \exp \right), \text { hence } \\
& t_{z}: \mathcal{O}\left(k_{x}^{n} ; \exp \right) \cong \mathcal{O}\left(k_{x}^{n} ; \exp \right) \\
& t_{z}: \mathcal{O}\left(k_{x}^{n} ; \text { infexp }\right) \cong \mathcal{O}\left(k_{x}^{n} ; \text { infexp }\right)
\end{aligned}
$$

Corollary 3.4. The space $W_{z}=\mathcal{L}\left(k<s-z>^{\prime}\right)=e^{z \bullet x} W_{0}$ is also given as

$$
W_{z}=\left\{w \in \mathcal{O}\left(k_{x}^{n}\right) ; \forall \delta>0 \exists C_{\delta}>0 \text { with }|w(x)| \leq C_{\delta} e^{\Re(z \bullet x)+\delta\|x\|}\right\} .
$$

Proof. The following equivalences hold:

$$
\begin{gathered}
w \in W_{z} \Longleftrightarrow e^{-z \bullet x} w(x) \in W_{0}=\mathcal{O}\left(k_{x}^{n} ; \text { infexp }\right) \Longleftrightarrow \\
\forall \delta>0 \exists C_{\delta}>0 \text { with } e^{-\Re(z \bullet x)}|w(x)|=\left|e^{-z \bullet x} w(x)\right| \leq C_{\delta} e^{\delta|| x||} \Longleftrightarrow \\
\forall \delta>0 \exists C_{\delta}>0 \text { with }|w(x)| \leq C_{\delta} e^{\Re(z \bullet x)+\delta|| x||} .
\end{gathered}
$$


We finally determine the module structures of the considered spaces. The Laplace transform isomorphism

$$
\mathcal{L}: \mathcal{O}\left(k_{s}^{n}\right)^{\prime} \rightarrow\left(\mathcal{O}\left(k_{x}^{n} ; \exp \right), \circ_{0}\right) \text { is } \mathcal{O}\left(k_{s}^{n}\right) \text {-linear and } k<s-z>^{\prime} \subset \mathcal{O}\left(k_{s}^{n}\right)^{\prime}
$$

is an $\mathcal{O}\left(k_{s}^{n}\right)$-submodule. Therefore

$$
\begin{gathered}
W_{z}=\mathcal{L}\left(k<s-z>^{\prime}\right) \subset\left(\mathcal{O}\left(k_{x}^{n} ; \exp \right), \circ_{0}\right) \text { is an } \mathcal{O}\left(k_{s}^{n}\right) \text {-submodule too with } \\
\quad f \circ_{0} w, f \in \mathcal{O}\left(k_{s}^{n}\right), w \in W_{z}, \text { especially } \\
s^{\mu} \circ_{0} w=\partial^{\mu} w,\left(e^{y \bullet s} \circ_{0} w\right)(x)=t_{-y}(w)(x)=w(x+y), x, y \in k^{n} .
\end{gathered}
$$

But the dual module $k\langle s-z\rangle^{\prime}$ is also a $k\langle s-z>$-module with $(f \circ \psi)(g):=$ $\psi(f g)$ which for $f \in \mathcal{O}\left(k_{s}^{n}\right)$ coincides with the structure as $\mathcal{O}\left(k_{s}^{n}\right)$-module. Via $\mathcal{L}$ we transport this structure to $W_{z}$ and obtain a scalar multiplication

$f \circ_{z} w, f \in k\langle s-z\rangle, w \in W_{z}$, with $f \circ_{z} w=f \circ_{0} w$ for $f \in \mathcal{O}\left(k_{s}^{n}\right)$, especially $s^{\mu} \circ_{0} w=\partial^{\mu} w,\left(e^{y \bullet s} \circ_{0} w\right)(x)=t_{-y}(w)(x)=w(x+y), x, y \in k^{n}$, according to (43).

Corollary 3.5. With these structures the k-isomorphisms from (41)

$$
t_{-z}^{\prime}: k<s>^{\prime} \rightarrow k<s-z>^{\prime} \text { and } e^{z \bullet x} \cdot: W_{0} \rightarrow W_{z}
$$

are semilinear with respect to $t_{z}: k<s>\cong k<s-z>, f \mapsto f(s-z)$, where

$$
\begin{gathered}
f=\sum_{\mu \in \mathbb{N}^{n}} f_{\mu} s^{\mu} \in k<s>, t_{z}(f)=\sum_{\mu \in \mathbb{N}^{n}} f_{\mu}(s-z)^{\mu} \in k<s-z>\text {, i.e., } \\
t_{-z}^{\prime}(f \circ \varphi)=t_{z}(f) \circ t_{-z}^{\prime}(\varphi), f \in k<s>, \varphi \in k<s>^{\prime} \text {, and } \\
e^{z \bullet x}\left(f \circ \circ_{0} w\right)=t_{z}(f) \circ_{z}\left(e^{z \bullet x} w\right), f \in k<s>, w \in W_{0}=\mathcal{O}\left(k_{x}^{n} ; \text { infexp }\right), \text { or } \\
f \circ_{z} w=e^{z \bullet x}\left(t_{-z}(f) \circ_{0}\left(e^{-z \bullet x} w\right)\right), f \in k<s-z>, w \in W_{z} .
\end{gathered}
$$

These formulas generalize the standard formula $(\partial-z)^{\mu}\left(e^{z \bullet x} w\right)=e^{z \bullet x} \partial^{\mu} w$.

Proof. Due to (41) and preceding structure $\circ_{z}$ of $W_{z}$ it suffices to show this for $t_{-z}^{\prime}$. But for $f \in k<s>$ and $g \in k\langle s-z>$ we get

$$
\begin{gathered}
t_{-z}^{\prime}(f \circ \varphi)(g)=(f \circ \varphi)\left(t_{-z}(g)\right)=\varphi\left(f t_{-z}(g)\right)= \\
\varphi t_{-z}\left(t_{z}(f) g\right)=\left(t_{z}(f) \circ t_{-z}^{\prime}(\varphi)\right)(g) \Longrightarrow t_{-z}^{\prime}(f \circ \varphi)=t_{z}(f) \circ t_{-z}^{\prime}(\varphi) .
\end{gathered}
$$

\section{Analytic local algebras}

Let $k$ be the field of real or of complex numbers. An analytic local algebra [14, Kap. II] is a nonzero factor algebra

$$
\begin{gathered}
A=k<s>/ \mathfrak{a}, \mathfrak{a} \subsetneq k<s>\text {, where } \\
k<s>=k<s_{1}, \cdots, s_{n}>, n>0, \text { and } \mathfrak{m}=\sum_{i=1}^{n} k<s>s_{i}
\end{gathered}
$$

are the algebra of convergent power series as discussed in Section 3 and its maximal ideal. The algebra $A$ is local with the unique maximal ideal $\mathfrak{m}_{A}:=\mathfrak{m} / \mathfrak{a}$ and the residue field $k=A / \mathfrak{m}_{A}$. Obviously $A$ is also noetherian. The sequence topology on $k<s>$ induces the final topology on $A=k<s>/ \mathfrak{a}$ which is 
called the sequence topology on $A$. Each $k$-algebra homomorphism between analytic local $k$-algebras is continuous [14, Satz II.1.4 on p.84]. Like $k\langle s\rangle$ the algebra $A$ is a Hausdorff, locally convex, sequence-complete topological algebra [14, Satz II.1.7 on p.85]. Let $A^{\prime}$ be the dual space of continuous $k$-linear functions from $A$ to $k$. Since $A$ is a topological algebra $A^{\prime}$ is an $A$-module via the standard scalar multiplication $\circ$ defined by

$$
(f \circ \varphi)(g):=\varphi(f g), f, g \in A, \varphi \in A^{\prime} .
$$

We are going to show that ${ }_{A} A^{\prime}$ is an injective cogenerator. We prepare this result by a more general theorem on injective cogenerators.

Let $A$ be a commutative noetherian $k$-algebra with a topology. We call an $A$ module $M$ topological if it is a locally convex vector space and if the multiplications $A \rightarrow M, a \mapsto a m, m \in M$, are continuous [6, Lemma 2.19]. Assume now that ${ }_{A} A$ is a topological module in this sense (We do not need or require that $A$ is a topological algebra). Then all f.g. free modules $A^{1 \times q}$ are topological $A$-modules with the product topology and all linear maps $\circ R: A^{1 \times k} \rightarrow A^{1 \times q}, R \in A^{k \times q}$, are continuous. We study the category ${ }_{A} \mathbf{M o d}^{f}$ of all f.g. or noetherian $A$ modules. Any linear map $f: M_{1} \rightarrow M_{2}$ between f.g. $A$-modules can be embedded into a commutative diagram with exact rows

$$
\begin{array}{cccccc}
A^{1 \times k_{1}} & \stackrel{\circ R_{1}}{\longrightarrow} & A^{1 \times q_{1}} & \stackrel{\nu_{1}}{\longrightarrow} & M_{1} \cong A^{1 \times q_{1}} / A^{1 \times k_{1}} R_{1} & \rightarrow 0 \\
\downarrow \circ S & & \downarrow \circ T & & \downarrow f & \\
A^{1 \times k_{2}} & \stackrel{\circ R_{2}}{\longrightarrow} & A^{1 \times q_{2}} & \stackrel{\nu_{2}}{\longrightarrow} & M_{2} \cong A^{1 \times q_{2}} / A^{1 \times k_{1}} R_{2} & \rightarrow 0
\end{array}
$$

With the final quotient topology the modules $M_{i}$ become topological $A$-modules in the above sense such that the epimorphisms $\nu_{i}$ are strict or, equivalently, open. Since $f \nu_{1}=\nu_{2}(\circ T)$ is continuous and $\nu_{1}$ is open we infer that $f$ is continuous too. For any module $M \in{ }_{A} \mathbf{M o d}^{f}, M_{1}:=M_{2}:=M$ and $f:=\operatorname{id}_{M}$ we infer that $\operatorname{id}_{M}$ is a topological isomorphism. This signifies that the topology of $M$ does not depend on the choice of the representation $M \cong A^{1 \times q} / A^{1 \times k} R$. We call this topology the canonical topology of the f.g. $A$-module $M$ and use this in the sequel. Then every $A$-epimorphism in ${ }_{A} \mathbf{M o d}{ }^{f}$ is open. For every $M \in{ }_{A} \mathbf{M o d}^{f}$ we also consider the dual space $M^{\prime}$ which is again an $A$-module as in (46). There result two contravariant left exact functors

$$
(-)^{\prime}, \operatorname{Hom}_{A}\left(-, A^{\prime}\right):{ }_{A} \mathbf{M o d}^{f} \rightarrow{ }_{A} \mathbf{M o d}
$$

and the functorial homomorphism

$$
\epsilon_{M}: M^{\prime} \rightarrow \operatorname{Hom}_{A}\left(M, A^{\prime}\right), \varphi \mapsto \phi, \phi(m)(f):=\varphi(f m),
$$

which for $M=A$ and then $M=A^{1 \times q}$ is obviously an isomorphism. The exact sequence $A^{1 \times k} \stackrel{\circ R}{\longrightarrow} A^{1 \times q} \stackrel{\nu}{\longrightarrow} M \rightarrow 0$ induces the commutative diagram with exact rows

$$
\begin{aligned}
& 0 \rightarrow \quad \begin{array}{ccccc}
M^{\prime} & \nu^{\prime}=\operatorname{Hom}(\nu, k) & A^{\prime q}=\left(A^{1 \times q}\right)^{\prime} & \stackrel{R \circ}{\longrightarrow} & A^{\prime k} \\
\downarrow \epsilon_{M} & & \downarrow \epsilon_{A^{1 \times q}} & & \downarrow \epsilon_{A^{1 \times k}}
\end{array} \\
& 0 \rightarrow \operatorname{Hom}_{A}\left(M, A^{\prime}\right) \quad \stackrel{\operatorname{Hom}\left(\nu_{2} A^{\prime}\right)}{\longrightarrow} \quad \operatorname{Hom}_{A}\left(A^{1 \times q}, A^{\prime}\right) \stackrel{\operatorname{Hom}\left(\circ R, A^{\prime}\right)}{\longrightarrow} \quad \operatorname{Hom}_{A}\left(A^{1 \times k}, A^{\prime}\right)
\end{aligned}
$$

where the exactness of the first row follows from the openness of $\nu$ and where the two right vertical maps are isomorphisms. This implies that also $\epsilon_{M}$ is bijective, i.e., $\epsilon_{M}$ is a functorial isomorphism on the category ${ }_{A} \mathbf{M o d}^{f}$.

Lemma 4.1. Assume that $A$ is a noetherian and that ${ }_{A} A$ is a topological $A$ module as introduced above. 
1. If for each ideal $\mathfrak{a}$ of $A$ the canonical topology of $\mathfrak{a}$ coincides with the induced topology from $A$ then ${ }_{A} A^{\prime}$ is injective.

2. If in addition to 1. A is Hausdorff and each maximal ideal is closed then ${ }_{A} A^{\prime}$ is also a cogenerator.

Proof. 1. Let $\mathfrak{a}$ be an ideal of $A$. It is f.g. and thus carries the canonical topology and the induced topology from $A$ which coincide by assumption. The space $\mathfrak{a}^{\prime}$ is the dual space with respect to this topology. By assumption $A$ is a locally convex space. The Hahn-Banach theorem implies that any function in $\mathfrak{a}^{\prime}$ can be extended to a function in $A^{\prime}$ or, in other words, the restriction map $A^{\prime} \rightarrow \mathfrak{a}^{\prime}$ is surjective. Since $\epsilon_{M}$ is a functorial isomorphism also

$$
\operatorname{Hom}_{A}\left(\operatorname{inj}, A^{\prime}\right): \operatorname{Hom}_{A}\left(A, A^{\prime}\right) \rightarrow \operatorname{Hom}_{A}\left(\mathfrak{a}, A^{\prime}\right), \phi \mapsto \phi \mid \mathfrak{a}
$$

is surjective. From Baer's criterion for injectivity we infer that $A^{\prime}$ is injective. 2. Since $A^{\prime}$ is injective its cogenerator property can be inferred if each simple module can be embedded into $A^{\prime}$. So consider any simple module $A / \mathfrak{m}$ where $\mathfrak{m}$ is a maximal ideal of $A$. By assumption $\mathfrak{m}$ is closed and $A$ is Hausdorff, hence also $A / \mathfrak{m}$ is a nonzero locally convex Hausdorff space. Again by the Hahn-Banach theorem we conclude $0 \neq(A / \mathfrak{m})^{\prime} \cong \operatorname{Hom}_{A}\left(A / \mathfrak{m}, A^{\prime}\right)$. Hence there is nonzero $A$-linear map $A / \mathfrak{m} \rightarrow A^{\prime}$ which is a monomorphism since $A / \mathfrak{m}$ is simple.

We return to an analytic local algebra $A$ as introduced above. Its quoted properties imply that ${ }_{A} A$ is a topological module as used in Lemma 4.1. The modules in ${ }_{A} \mathbf{M o d}^{f}$ are called analytic modules in [14, Kap.II]. Their canonical topology is again called the sequence topology. The next result shows that the assumptions of Lemma 4.1 are satisfied for $A$.

Result 4.2. Consider an analytic local algebra $A$ with its sequence topology and its category ${ }_{A} \mathbf{M o d}^{f}$ of analytic modules with their canonical or sequence topology. These modules have the following properties:

1. The algebra $A$ is even a topological algebra and any ${ }_{A} M$ is a topological module in the sense that the multiplication $A \times M \rightarrow M$ is continuous [14, Satz II.1.10].

2. Each submodule of a module $M \in{ }_{A} \mathbf{M o d}^{f}$ is closed and especially each module $M$ is Hausdorff [14, Satz II.1.10 on p.87].

3. Any $M \in{ }_{A} \mathbf{M o d}^{f}$ is locally convex [14, Satz I.8.7 on p.74 and Satz and Bemerkung on p.86].

4. The sequence topology of a submodule $N$ of $M \in{ }_{A} \mathbf{M o d}^{f}$ coincides with the topology induced from the sequence topology of M [14, Satz II.2.9 on p.97].

Theorem 4.3. For any analytic local algebra $A$ over $k=\mathbb{R}$ or $k=\mathbb{C}$ and its dual space $A^{\prime}$ with the canonical $A$-structure the module ${ }_{A} A^{\prime}$ is an injective cogenerator.

In particular this holds for the algebra $k<s-z>=k<s_{1}-z_{1}, \cdots, s_{n}-$ $z_{n}>, z \in k^{n}$, of convergent power series around $z \in k^{n}$, and the module $W_{z}:=\mathcal{O}\left(k_{x}^{n} ;\right.$ infexp $) e^{z \bullet x} \cong k<s-z>^{\prime}$ with its canonical structure, compare Cor. 3.4 and 3.5 .

Proof. Result 4.2 implies that the assumptions of Lemma 4.1 are satisfied for analytic local algebras $A$. 


\section{Holomorphic functions on compact Stein sets}

In this section we prove the injective cogenerator property of ${ }_{A} A^{\prime}$ where $A$ is the ring of holomorphic functions on a connected, semi-analytic compact Stein subset of a complex manifold $Z$. Such an $A$ is noetherian. In the standard cases we give concrete representations of $A^{\prime}$ of systems theoretic interest.

In contrast to Sections 4 and 6 we have to use the full theory and formalism of coherent sheaves on a complex space and not only the algebraic consequences of this theory. We give exact quotations for all deep results which we use. Lack of space prevents more detailed explanations of these results.

Let $Z$ be a reduced complex space [16, p.17-22] or holomorphic variety [29, Def. 7.6.4] with its sheaf $\mathcal{O}:=\mathcal{O}_{Z}$ resp. algebra $\mathcal{O}(Z)$ of holomorphic resp. global holomorphic functions. It is always assumed that $Z$ has a countable basis of the topology. For every compact subset $K$ of $Z$ the closed set

$$
\widehat{K}_{Z}:=\left\{z \in Z ; \forall f \in \mathcal{O}_{Z}(Z) ;|f(z)| \leq \max _{x \in K}|f(x)|\right\}
$$

is called the holomorphically convex hull of $K$ in $Z$ [16, Def. IV.2.1], [29, Def. 10.6.1]. The space $Z$ is called holomorphically convex if the hull $\widehat{K}_{Z}$ of every compact set $K$ is compact. Stein spaces $Z$ are defined by the property that Cartan's Theorems A and B hold for coherent analytic sheaves on $Z$ [16, Kap. IV,V], [17, Ch. I] and [29, Ch.10 ]. A compact subset of a complex space is called Stein if Cartan's Theorems A and B hold for coherent analytic sheaves on $K[16$, Def. in $\S$ III.3.1, Def. IV.1.1, Satz IV.1.1, Satz IV.1.2]. The definition in [29, p.282, Cor. 11.5.4] differs from this. The usual convexity in a real vector space, for instance in $\mathbb{C}^{n}=\mathbb{R}^{2 n}$, is called geometric convexity here. A quadrangle of $\mathbb{C}^{n}=\mathbb{R}^{2 n}$ is a cartesian product of $2 n$ non-empty intervals of $\mathbb{R}[16, p .93]$ and especially geometrically convex. It is compact if all these intervals are closed and finite. A subset of $\mathbb{C}^{n}$ is, roughly, called semi-analytic $[10, p .120]$ if in a neighborhood of every point it can be described by finitely many inequalities of real-analytic functions.

If $K$ is a subset of $Z$ any section $t \in \mathcal{O}(K)$, i.e., any holomorphic function on $K$, can be extended to an open neighborhood $U$ of $K$, i.e, there is $s \in \mathcal{O}(U)$ with $s \mid K=t$ [15, Satz A.4.1]. This implies the algebraic isomorphism

$$
\mathcal{O}(K) \cong \lim _{U} \mathcal{O}(U) \ni f_{K}, f \in \mathcal{O}(U),
$$

where $U$ runs over the downward directed set of open neighborhoods of $K$ and where $f_{K}$ denotes the equivalence class of $f \in \mathcal{O}(U)$ in the direct limit. The $\mathcal{O}(U)$ are Fréchet algebras [16, Satz V.6.8] with the topology of compact convergence and especially locally convex. The topology of $\mathcal{O}(U)$ is given by the semi-norms

$$
\|f\|_{C}:=\max _{z \in C}|f(z)|, f \in \mathcal{O}(U), C \subset U, C \text { compact. }
$$

The algebra $\mathcal{O}(K)$ carries its unique locally convex inductive limit topology [5, p. II.29].

Result 5.1. Let $Z$ be a reduced complex space and $K$ a compact subset of $Z$.

1. The space $Z$ is Stein if and only if it is holomorphically convex and if global holomorphic functions separate the points of $Z$, i.e. for $z_{1} \neq z_{2}$ in $Z$ there is $f \in \mathcal{O}(Z)$ with $f\left(z_{1}\right) \neq f\left(z_{2}\right)$ [16, Satz V.4.3], [29, Def. 10.6.2, Ex. 11.13 on p.310]. A holomorphically convex open and any closed complex subspace or holomorphic subvariety of a Stein space are Stein [16, Satz V.1.1]. The prototypical Stein space is $\mathbb{C}^{n}, n>0$. Any open quadrangle, open polydisc or open geometrically convex subset in $\mathbb{C}^{n}$ is holomorphically convex and thus Stein [16, p.114]. 
2. If $Z$ is Stein and $K=\widehat{K}_{Z}$ then $K$ is Stein in $Z$ [29, Cor. 11.5.4].

3. If $K \subset \mathbb{C}^{n}=\mathbb{R}^{2 n}$ is geometrically convex then $K=\widehat{K}_{\mathbb{C}^{n}}$ [20, §3.1, Cor. 1], [19, Th. 2.7.7] and hence $K$ is Stein.

4. If $Z$ is Stein and $K=\widehat{K}_{Z}$ then $K$ has a neighborhood basis of open OkaWeil domains $U$ [17, Def. I.10]: These $U$ are Stein by themselves and the restriction $\mathcal{O}(Z) \rightarrow \mathcal{O}(U)$ has a dense image [17, Th. I.11, Cor. I.16]. If $K$ is connected then $U$ can also be assumed connected.

5. If $K$ is semi-analytic and Stein in $Z$ then $\mathcal{O}(K)$ is noetherian [10, Th. I.9]). Reducedness of $Z$ is not required in Frisch's theorem.

6. If $Z$ is Stein and if $K \subset Z$ is a compact analytic polyhedron of the form

$$
K=\bigcap_{j=1}^{N}\left\{z \in Z ;\left|f_{j}(z)\right| \leq 1\right\}, f_{j} \in \mathcal{O}(Z), j=1, \cdots, N,
$$

then $K=\widehat{K}_{Z}$ by (51) and (54) and $K$ is semi-analytic. By items (2) and (5) $K$ is Stein and $\mathcal{O}(K)$ is noetherian. The prototypical case is a compact polydisc $K$ of $\mathbb{C}^{n}$.

7. If $K \subset \mathbb{C}^{n}$ is geometrically convex and semi-analytic then items (3), (2), (4) and (5) apply to $K$. If $K$ is a compact polyhedron, i.e., the intersection of finitely many closed half-spaces, then it is semi-analytic. A compact polydisc is semi-analytic and geometrically convex, but not an intersection of finitely many closed half-spaces.

Lemma 5.2. If $K$ is a compact subset of the Stein space $Z$ with $K=\widehat{K}_{Z}$ the image of the canonical map $\operatorname{can}_{K}: \mathcal{O}(Z) \rightarrow \mathcal{O}(K), f \mapsto f_{K}$, is dense and hence the adjoint $\mathcal{O}(K)^{\prime} \rightarrow \mathcal{O}(Z)^{\prime}$ is injective. According to Result 5.1,(3),(4), this property holds for $Z=\mathbb{C}^{n}$ and a geometrically convex compact subset $K \subset$ $\mathbb{C}^{n}$.

Proof. According to Result 5.1,(4), we may and do only use open neighborhoods $U$ of $K$ in the direct limit for which the restriction $\mathcal{O}(Z) \rightarrow \mathcal{O}(U), h \mapsto h \mid U$, has a dense image. Let $f_{K} \in \mathcal{O}(K), f \in \mathcal{O}(U)$, be any element of $\mathcal{O}(K)$ and $V_{1}$ any neighborhood of 0 in $\mathcal{O}(K)$. Since $\mathcal{O}(U) \rightarrow \mathcal{O}(K), g \mapsto g_{K}$, is continuous $V_{2}:=\left\{g \in \mathcal{O}(U) ; g_{K} \in V_{1}\right\}$ is a neighborhood of 0 in $\mathcal{O}(U)$. Since $\mathcal{O}(Z) \rightarrow \mathcal{O}(U)$ has a dense image there is $h \in \mathcal{O}(Z)$ such that

$f-(h \mid U) \in V_{2}$ and thus $f_{K}-(h \mid U)_{K}=f_{K}-h_{K} \in V_{1}$ and $h_{K} \in f_{K}+V_{1}$.

But this signifies that the $h_{K}, h \in \mathcal{O}(Z)$, form a dense subset of $\mathcal{O}(K)$.

From now on we assume that $Z$ is a complex manifold [16], [15] and that $K$ is a compact, connected, semi-analytic Stein subset of $Z$ so that $A:=\mathcal{O}(K)$ is noetherian. The prototypical cases are exposed in Result 5.1, items (6) and (7). The space $Z$ is locally compact, metrizable and locally path-connected, in particular connected components are open and closed. For $z \in Z$ let $\mathcal{O}_{z}$ be the algebra of germs of holomorphic functions near $z$. It is an analytic local algebra with its sequence topology. Since $Z$ is a manifold $\mathcal{O}_{z}$ is an algebra of convergent power series as in Section 3, up to isomorphism. All canonical maps

$$
\operatorname{can}_{z}: \mathcal{O}(U) \rightarrow \mathcal{O}_{z}, f \mapsto f_{z}, z \in U \subseteq Z, U \text { open, }
$$

are continuous [15, Th. V.6.4, V.6.5]. If $U$ is connected then the identity theorem holds for $\mathcal{O}(U)$, i.e., all canonical maps from (55) are injective. If $\emptyset \neq V \subset$ $U$ are open sets the restriction $\mathcal{O}(U) \rightarrow \mathcal{O}(V)$ is continuous. If in addition $U$ 
is connected this restriction is injective by the identity theorem and we identify $\mathcal{O}(U) \subset \mathcal{O}(V)$.

Since $K$ is compact and connected standard topological arguments furnish open neighborhoods $U_{i}, i \in \mathbb{N}$, of $K$ with the following properties:

1. For all $i \in \mathbb{N}$ the set $U_{i}$ is connected, its closure $\overline{U_{i}}$ is compact and $\overline{U_{i}} \subset$ $U_{i-1}$.

2. The decreasing sequence of $U_{i}$ is a basis of open neighborhoods of $K$, i.e., each such neighborhood contains some $U_{i}$, especially $K=\bigcap_{i=1}^{\infty} U_{i}$.

From these neighborhoods we derive the algebras

$$
\begin{gathered}
B_{i}:=\mathcal{O}\left(U_{i}\right) \bigcap \mathrm{C}^{0}\left(\overline{U_{i}}\right) \subset A_{i}:=\mathcal{O}\left(U_{i}\right) \text { with } B_{i} \subset A_{i} \subset B_{i+1} \subset A_{i+1}, \\
A=\bigcup_{i=1}^{\infty} A_{i}=\lim _{\longrightarrow} A_{i}=\bigcup_{i=1}^{\infty} B_{i}={\underset{\lim }{\longrightarrow} B_{i}}^{\longrightarrow}
\end{gathered}
$$

with the following specifications: $\mathrm{C}^{0}\left(\overline{U_{i}}\right)$ is the Banach algebra of continuous functions on the compact set $\overline{U_{i}}$ with the maximum norm $\|f\|_{\overline{U_{i}}}$ and so is $B_{i}$. The $A_{i}$ are Fréchet algebras with the topology of compact convergence. Since the $U_{i}$ are connected the identity theorem implies $A_{i} \subset A_{i+1}$ and then also all other inclusions in (56). All inclusions are continuous. Since the $U_{i}$ are a basis of open neighborhoods of $K$ we infer that each $\mathcal{O}(U)$ from (52) maps into some $A_{i}$ from which the identities in the second row of (56) including the locally convex inductive limit topologies follow. For each $z \in U_{i}$ there is the continuous injective canonical map $\operatorname{can}_{z} \mid A_{i}: A_{i}=\mathcal{O}\left(U_{i}\right) \rightarrow \mathcal{O}_{z}$ between locally convex spaces from (55). By definition of the inductive limit it implies the continuous injective canonical maps

$$
\operatorname{can}_{z}: A=\bigcup_{i=1}^{\infty} A_{i} \rightarrow \mathcal{O}_{z}, z \in K, \text { and } \mathcal{O}(Z) \rightarrow A=\mathcal{O}(K), f \mapsto f_{K}
$$

Lemma 5.3. Assume that $K$ is a compact, connected, semi-analytic Stein subset of the complex manifold $Z$ so that $A:=\mathcal{O}(K)$ is noetherian. The algebra $A$ is endowed with the inductive limit topology from above. Then each ideal of $A$ is closed and $A$ is Hausdorff (since $\{0\}$ is closed).

Proof. 1. We use the data from (56) and (57). Since the $A_{i}$ are topological algebras they are topological modules over themselves in the sense of Section 4 . Therefore also ${ }_{A} A$ is a topological module and all maps $\circ R: A^{1 \times k} \rightarrow A^{1 \times q}, R \in A^{k \times q}$, are continuous.

2. Let $\mathfrak{a}$ be any ideal of $A$ and $\overline{\mathfrak{a}}$ its closure. Since ${ }_{A} A$ is a topological module $\overline{\mathfrak{a}}$ is also an ideal. We want to show that $\mathfrak{a}=\overline{\mathfrak{a}}$. By diminishing $Z$ we may and do assume that $Z=U_{1}$ and that there are matrices $R \in A_{1}^{k \times 1}$ and $R^{\prime}=\left(\begin{array}{c}R \\ R_{2}\end{array}\right) \in$ $A_{1}^{k^{\prime} \times 1}$ such that

$$
\mathfrak{a}=A^{1 \times k} R \subseteq \overline{\mathfrak{a}}=A^{1 \times k^{\prime}} R^{\prime} .
$$

We define the coherent ideals $\mathfrak{A} \subseteq \mathcal{O}$ and $\mathfrak{A}^{\prime} \subseteq \mathcal{O}$ by

$$
\begin{gathered}
\mathfrak{A}:=\operatorname{im}\left(\mathcal{O}^{1 \times k} \stackrel{\circ R}{\longrightarrow} \mathcal{O}\right) \subseteq \mathfrak{A}^{\prime}:=\operatorname{im}\left(\mathcal{O}^{1 \times k^{\prime}} \stackrel{\circ R^{\prime}}{\longrightarrow} \mathcal{O}\right) \text {. Then } \\
\mathfrak{A}_{z}=\mathcal{O}_{z}^{1 \times k} R_{z} \subseteq \mathfrak{A}_{z}^{\prime}=\mathcal{O}_{z}^{1 \times k^{\prime}} R_{z}^{\prime}, z \in Z .
\end{gathered}
$$

Since $K$ is Stein in $Z$ the important Theorem B holds [15, Satz IV.1.1]: The maps

$$
A^{1 \times k}=\mathcal{O}(K)^{1 \times k} \stackrel{\circ R}{\longrightarrow} \mathfrak{A}(K) \text { and } A^{1 \times k^{\prime}}=\mathcal{O}(K)^{1 \times k^{\prime}} \stackrel{\circ R^{\prime}}{\longrightarrow} \mathfrak{A}^{\prime}(K)
$$


are surjective, i.e., $\mathfrak{a}=\mathfrak{A}(K)$ and $\overline{\mathfrak{a}}=\mathfrak{A}^{\prime}(K)$. Moreover

$$
\mathfrak{A}_{z}=\mathcal{O}_{z}^{1 \times k} R_{z}=\mathcal{O}_{z} \operatorname{can}_{z}(\mathfrak{a}) \subseteq \mathfrak{A}_{z}^{\prime}=\mathcal{O}_{z}^{1 \times k^{\prime}} R_{z}^{\prime}=\mathcal{O}_{z} \operatorname{can}_{z}(\overline{\mathfrak{a}}), z \in K .
$$

For $z \in K$ the canonical map $\operatorname{can}_{z}: A \rightarrow \mathcal{O}_{z}$ is continuous. Hence

$$
\operatorname{can}_{z}(\overline{\mathfrak{a}}) \subseteq \overline{\operatorname{can}_{z}(\mathfrak{a})} \subseteq \overline{\mathcal{O}_{z} \operatorname{can}_{z}(\mathfrak{a})}=\mathcal{O}_{z} \operatorname{can}_{z}(\mathfrak{a}), z \in K
$$

The last equality follows from the fact that in the sequence topology all ideals of $\mathcal{O}_{z}$ are closed; compare Result 4.2,(2). We conclude

$$
\begin{gathered}
\mathfrak{A}_{z}^{\prime}=\mathcal{O}_{z} \operatorname{can}_{z}(\overline{\mathfrak{a}})=\mathcal{O}_{z} \operatorname{can}_{z}(\mathfrak{a})=\mathfrak{A}_{z}, z \in K \Longrightarrow \\
\mathfrak{A}\left|K=\mathfrak{A}^{\prime}\right| K \Longrightarrow \mathfrak{a}=\mathfrak{A}(K)=\mathfrak{A}^{\prime}(K)=\overline{\mathfrak{a}} .
\end{gathered}
$$

Using substantial results from Functional Analysis we are going to verify the two assumptions of Lemma 4.1 for the ring $A$ from Lemma 5.3 and can then infer that ${ }_{A} A^{\prime}$ is an injective cogenerator.

A Fréchet space is called a Montel space if each bounded subset has a compact closure. The space $\mathcal{O}(Z)$ of holomorphic functions on the complex manifold with its topology of compact convergence is a Montel space [29, Th. 11.2.5] and the same holds for all $A_{i}=\mathcal{O}\left(U_{i}\right)$ from above. A continuous linear map $f: V \rightarrow W$ of locally convex spaces is called compact if the image $f(X)$ of any bounded subset $X \subseteq V$ has a compact closure $\overline{f(X)}=\mathrm{cl}_{W}(f(X))$ in $W$.

From (56) we know that $A=\bigcup_{i=1}^{\infty} B_{i}=\lim B_{i}$ is the inductive limit of the Banach spaces $B_{i}$. Such a limit is called a DFS (Dual Fréchet-Schwartz)-space if the inclusions $B_{i} \subset B_{i+1}$ are compact [21, Def. A.5.10].

Lemma 5.4. The inclusions $B_{i} \rightarrow B_{i+1}$ from (56) are compact and therefore $A$ is a DFS-space.

Proof. Consider the continuous inclusions $\gamma: B_{i} \stackrel{\alpha}{\longrightarrow} A_{i} \stackrel{\beta}{\longrightarrow} B_{i+1}$ from (56) and let $X \subseteq B_{i}$ be bounded. Since continuous linear maps preserve boundedness $X=\alpha(X)$ is bounded in $A_{i}$. Since $A_{i}$ is a Montel space the closure $\operatorname{cl}_{A_{i}}(X) \subseteq$ $A_{i}$ is compact and has a compact and thus closed image

$\beta\left(\operatorname{cl}_{A_{i}}(X)\right)=\operatorname{cl}_{A_{i}}(X)$ in $B_{i+1}$. But then $\gamma(X)=X \subseteq \operatorname{cl}_{B_{i+1}}(X) \subseteq \operatorname{cl}_{A_{i}}(X)$. Hence $\operatorname{cl}_{B_{i+1}}(\gamma(X))=\operatorname{cl}_{B_{i+1}}(X)$ is compact. This signifies the compactness of $\gamma$.

Each ideal $\mathfrak{a}$ of $A$ is closed by Lemma 5.3 and therefore also a DFS-space with the induced topology from $A$ [21, Th. A.5.13], i.e., $\mathfrak{a}$ is a locally convex inductive limit of Banach spaces.

Lemma 5.5. If $f: V \rightarrow W$ is a continuous epimorphism of DFS-spaces and if $W$ is Hausdorff then $f$ is strict and thus open.

Proof. This is a special case of [5, Cor. of Prop. II.4.10 on p.II.34].

Summing up we obtain the desired theorem.

Theorem 5.6. Assume that $K$ is a compact, connected, semi-analytic Stein subset of the complex manifold $Z$ so that $A:=\mathcal{O}(K)$ is noetherian by Result 5.1. The ring $A$ is furnished with its locally convex inductive limit topology from above. Then ${ }_{A} A^{\prime}$ is an injective cogenerator. According to Result 5.1 the assumptions are satisfied for compact analytic polyhedra and compact semi-analytic and geometrically convex subsets $K \subset \mathbb{C}^{n}$. 
Proof. In Lemma 5.3 we have shown that all ideals of $A$ are closed. This especially implies condition 2. of Lemma 4.1. For an ideal $\mathfrak{a}=A^{1 \times k} R$ of $A$ we consider the factorization

$$
\circ R: A^{1 \times k} \stackrel{f:=(\circ R)_{\text {ind }}}{\longrightarrow} \mathfrak{a} \stackrel{\text { inj }}{\longrightarrow} A .
$$

where $\mathfrak{a}$ is endowed with the induced topology and all maps are continuous. But all spaces in this sequence are DFS-spaces and $f$ is continuous and surjective and therefore strict by Lemma 5.5. This signifies that the canonical topology of $\mathfrak{a}$, i.e., the final locally convex topology induced from the surjection $f$, coincides with the induced topology from $A$. Thus the assumptions of Lemma 4.1 are satisfied and consequently ${ }_{A} A^{\prime}$ is an injective cogenerator.

Theorem and Definition 5.7. $\quad 1$. In the situation of Th. 5.6 assume in addition that $Z$ is Stein and $K=\widehat{K}_{Z}$. Then the canonical map $\mathcal{O}(K)^{\prime} \rightarrow \mathcal{O}(Z)^{\prime}$ is injective by Lemma 5.2 and its image is the set of functionals $\phi \in \mathcal{O}\left(Z^{\prime}\right)$ with carrier K [19, Def. 4.5.1]: This signifies that for each open neighborhood $U$ of $K$ there is a constant

$$
M_{U}>0 \text { with }|\phi(f)| \leq M_{U} \sup _{z \in U}|f(z)| .
$$

2. Assume in addition that $Z=\mathbb{C}^{n}$ and that $K$ is a compact semi-analytic and geometrically convex subset of $\mathbb{C}^{n}$, for instance a compact polydisc or quadrangle. Let $W_{K} \subset \mathcal{O}\left(\mathbb{C}_{x}^{n}\right)$ denote the set of all entire functions $w(x)$ in $x=\left(x_{1}, \cdots, x_{n}\right)$ which satisfy the following growth condition: For each $\delta>0$ there is $C_{\delta}>0$ such that

$$
|w(x)| \leq C_{\delta} \exp \left(H_{K}(x)+\delta|x|\right), H_{K}(x)=\max _{s \in K} \Re(s \bullet x) .
$$

Here $|x|$ is any norm on $\mathbb{C}_{x}^{n}$. Then the Laplace transform induces the isomorphism

$$
\mathcal{L}: \mathcal{O}(K)^{\prime} \cong W_{K}, \varphi \mapsto \mathcal{L}(\varphi)=\varphi_{s}\left(e^{s \bullet x}\right) .
$$

Hence $W_{K}$ is an injective cogenerator over the noetherian ring $\mathcal{O}(K)$ with the unique action which extends the action $(37)$ of $\mathcal{O}\left(\mathbb{C}_{s}^{n}\right)$ on $\mathcal{O}\left(\mathbb{C}_{x}^{n} ; \exp \right)$.

Proof. 2. This follows directly from [19, Th. 4.5.3].

1. By (55) and Lemma 5.2 the map $\mathcal{O}(Z) \rightarrow A=\mathcal{O}(K), f \mapsto f_{K}$, is injective with a dense image and its adjoint

$$
\mathcal{O}(K)^{\prime} \rightarrow \mathcal{O}(Z)^{\prime}, \varphi \mapsto \phi, \phi(h):=\varphi\left(h_{K}\right),
$$

is injective. If $\varphi \in \mathcal{O}(K)^{\prime}$ and $U$ is any open neighborhood of $K$ the map

$$
\mathcal{O}(U) \rightarrow \mathcal{O}(K) \rightarrow \mathbb{C}, g \mapsto g_{K} \mapsto \varphi\left(g_{K}\right),
$$

is also continuous and hence there are a compact subset $K_{1} \subset U$ and $M>0$ with

$\left|\varphi\left(g_{K}\right)\right| \leq M \max _{z \in K_{1}}|g(z)|$ for all $g \in \mathcal{O}(U) \Longrightarrow$ with $\phi(h):=\varphi\left(h_{K}\right):$ $|\phi(h)|=\left|\varphi\left(h_{K}\right)\right| \leq M \max _{z \in K_{1}}|h(z)| \leq M \sup _{z \in U}|h(z)|$ for all $h \in \mathcal{O}(Z)$.

By definition $K$ is a carrier of $\phi \in \mathcal{O}(Z)^{\prime}$. Assume conversely that $K$ is a carrier for some $\phi \in \mathcal{O}(Z)^{\prime}$. Since $\mathcal{O}(Z) \rightarrow \mathcal{O}(K)$ is injective with a dense image the functional $\phi$ can be uniquely extended to $\varphi \in \mathcal{O}(K)^{\prime}$ if $\phi$ is continuous with respect to the topology induced from $\mathcal{O}(K)$ on $\mathcal{O}(Z)$. This continuity, however, follows from the carrier condition as before. 


\section{The case of Stein algebras}

Like the preceding section this one depends essentially on deep and difficult results on Stein spaces, Stein algebras and Stein modules over them. In Result 6.1 below we sum up results from the seminal paper [9] and book [16] and later use Result 6.1 only. The reader need not know the details of the difficult proofs of these results, especially no sheaf theoretic knowledge is required for the consequences which we draw. The method in this section are related to those in Sections 4 and 5. Complications come from the fact that the ring of operators is not noetherian anymore. The theory is valid over the complex field $\mathbb{C}$, and therefore all algebras and vector spaces in this section are defined over $\mathbb{C}$.

Let $\left(Z, \mathcal{O}_{Z}\right)$ be a Stein space with its sheaf of algebras $\mathcal{O}_{Z}=\left(\mathcal{O}_{Z}(U) ; U \stackrel{\text { open }}{\subset} Z\right)$ [16, §I.3, Kap.IV]. Although the algebras $\mathcal{O}_{Z}(U)$ may contain nilpotent elements its elements are called holomorphic functions on $U$. By definition the space $Z$ is Hausdorff, has a countable (basis of the) topology and is finite-dimensional.

Let $A=\mathcal{O}_{Z}(Z)$ denote its algebra of global sections or global holomorphic functions. It is called the Stein algebra of $Z$. More generally, any algebra $B$ algebraically isomorphic to such an $A=\mathcal{O}_{Z}(Z)$ is called a Stein algebra [16, §V.7, p.178]. For every $z \in Z$ there is again the fibre or stalk $\mathcal{O}_{z}$, i.e., the algebra of germs of holomorphic functions in $z$, with the canonical map $A \rightarrow \mathcal{O}_{z}, f \mapsto f_{z}$. The fibre $\mathcal{O}_{z}$ is an analytic local algebra in the sense of Section 4 (up to isomorphism) with its canonical sequence topology and its unique maximal ideal $\mathfrak{m}_{z}=\operatorname{ker}\left(\mathcal{O}_{z} \rightarrow \mathbb{C}, f_{z} \mapsto f(z)\right)$. Hence

$$
\begin{gathered}
\rho_{z}: A=\mathcal{O}_{Z}(Z) \rightarrow \mathcal{O}_{z} \rightarrow \mathbb{C}, f \mapsto f(z), \text { resp. its kernel } \\
\mathfrak{m}(z)=\operatorname{ker}\left(\rho_{z}\right)=\{f \in A ; f(z)=0\}
\end{gathered}
$$

are a character or algebra homomorphism from $A$ to $\mathbb{C}$ resp. a maximal ideal of A.

The most important example of a Stein complex space $Z$ for systems theory is, of course, the Stein manifold $\mathbb{C}^{n}$ with its sheaf $\mathcal{O}:=\mathcal{O}_{\mathbb{C}^{n}}$ (see Result 5.1). The Stein algebra $A$ of global sections is the algebra of entire holomorphic functions

$$
\begin{aligned}
A:=\mathcal{O}_{\mathbb{C}^{n}} & \left(\mathbb{C}^{n}\right)=\mathcal{O}\left(\mathbb{C}_{s}^{n}\right):=\left\{f: \mathbb{C}^{n} \rightarrow \mathbb{C}, s=\left(s_{1}, \cdots, s_{n}\right) \mapsto f(s), f \text { holomorphic }\right\} \\
=\left\{f=\sum_{\mu \in \mathbb{N}^{n}} f_{\mu} s^{\mu} \in \mathbb{C}[[s]] ; f \text { everywhere convergent }\right\}=\bigcap_{t \in \mathbb{R}_{+}^{n}} B_{t} &
\end{aligned}
$$

from Sections 3 and 5 . Due to the identity theorem we can and do identify functions and power series. With the corresponding identification the fibres $\mathcal{O}_{z}, z \in$ $\mathbb{C}^{n}$, have the form

$$
\mathcal{O}_{z}=\mathbb{C}<s-z>=\left\{\sum_{\mu \in \mathbb{N}^{n}} f_{\mu}(s-z)^{\mu} \text { convergent near } z \in \mathbb{C}^{n}\right\} .
$$

If $f \in A=\mathcal{O}\left(\mathbb{C}^{n}\right)$ then its germ in $z$ is identified with its Taylor series in $z$, i.e.,

$$
A=\mathcal{O}\left(\mathbb{C}_{s}^{n}\right) \rightarrow \mathcal{O}_{z}=\mathbb{C}<s-z>, f \mapsto f_{z}=\sum_{\mu \in \mathbb{N}^{n}} \frac{f^{(\mu)}(z)}{\mu !}(s-z)^{\mu}
$$

Let $\mathcal{M}$ denote a coherent $\mathcal{O}_{Z}$-module [16, Kap.A, §2]. Then the set $M:=\mathcal{M}(Z)$ of global sections is an $A=\mathcal{O}_{Z}(Z)$-module, and for each $z \in Z$ there are the fibre or stalk $\mathcal{M}_{z}$ and the canonical map $M \rightarrow \mathcal{M}_{z}, m \mapsto m_{z}$, where $m_{z}$ denotes the germ of $m$ at $z$. The fibre $\mathcal{M}_{z}$ is a f.g. $\mathcal{O}_{z}$-module, i.e., contained in the category 
$\mathcal{O}_{z} \operatorname{Mod}^{f}$ considered in Section 4. In particular, each $\mathcal{M}_{z}$ carries the sequence topology. The canonical map

$$
M_{z}:=\mathcal{O}_{z} \otimes_{A} M \rightarrow \mathcal{M}_{z}, f_{z} \otimes m \mapsto(f m)_{z}
$$

is an $\mathcal{O}_{z}$-isomorphism of f.g. $\mathcal{O}_{z}$-modules [9, Proof of Th. 2.1]. Therefore we can and do identify $M_{z}:=\mathcal{O}_{z} \otimes_{A} M=\mathcal{M}_{z}$ and $1 \otimes m=m_{z}$ and indeed do not use $\mathcal{M}$ anymore.

On $M$ there is a unique Fréchet topology such that all canonical maps

$$
M \rightarrow M_{z}=\mathcal{M}_{z}, m \mapsto m_{z},
$$

are continuous and this is called the canonical topology of $M$ [16, Satz V.6.4, Satz V.6.5] and always used in the sequel. Since $M$ is not f.g. in general its topology cannot simply be defined as a quotient topology from some $A^{1 \times q}$ as in Section 4 . In particular, $A$ is a Fréchet topological algebra, and indeed the canonical topology on $A$ is the unique topology on $A$ for which $A$ is a Fréchet topological algebra [16, p.187],[9, p.377-378]. (Algebraic) homomorphisms between Stein algebras are continuous [16, p.187]; especially all characters $\rho: A \rightarrow \mathbb{C}$ are continuous. If $Z$ is a Stein manifold or, more generally, a reduced Stein space then the canonical topology on $\mathcal{O}_{Z}(Z)$ is the topology of compact convergence [16, p.171 and Satz V.6.8]. This applies especially to $\mathcal{O}\left(\mathbb{C}_{s}^{n}\right)$.

The $A$-module $M=\mathcal{M}(Z)$ with its canonical topology is a topological $A$ module [9, §2, p.383]. Any $A$-linear map $\mathcal{M}_{1}(Z) \rightarrow \mathcal{M}_{2}(Z)$ between $A$-modules of global sections as above is continuous [9, Satz 2.1, Cor. 2.3]. An $A$-module $N$ which is algebraically $A$-isomorphic to a module $M=\mathcal{M}(Z)$ is called a Stein module over $A$. In general, it is not f.g. whereas all $M_{z}=\mathcal{O}_{z} \otimes_{A} M$ are f.g. It has a unique Fréchet topology such that $N$ with this topology is topologically $A$-isomorphic to some $M=\mathcal{M}(Z)$, and this topology is again called canonical and always used in the sequel. This is also the unique Fréchet topology on $N$ for which all canonical maps $N \rightarrow N_{z}, n \mapsto 1 \otimes n$, are continuous where the $N_{z}:=\mathcal{O}_{z} \otimes_{A} N \in \mathcal{O}_{z} \operatorname{Mod}^{f}$ carry the sequence topology. In particular, a Stein $A$-module is a topological $A$-module and all $A$-linear maps between Stein $A$-modules are continuous. Let

$$
{ }_{A} \operatorname{Mod}^{S t}\left({ }_{A} \operatorname{Mod}{ }^{S t, f}\right)
$$

denote the full subcategories of the category ${ }_{A} \operatorname{Mod}$ of all $A$-modules which consist of all (f.g.) Stein modules. We sum up the properties of $A$ and of ${ }_{A} \mathbf{M o d}^{S t}$ according to [9] and [16].

Result 6.1. ([9, §1,2], $[16, \S \mathrm{V} .6,7])$ Let $A=\mathcal{O}_{Z}(Z)$ be the Stein algebra of global sections of a Stein space $\left(Z, \mathcal{O}_{Z}\right)$, for instance $A=\mathcal{O}\left(\mathbb{C}_{s}^{n}\right)$, and ${ }_{A} \mathbf{M o d}^{S t} \subset_{A}$ Mod its full subcategory of Stein modules.

1. Finite direct sums (=products) of Stein modules are again such, especially all free modules $A^{1 \times q}, q \in \mathbb{N}$, belong to ${ }_{A} \mathbf{M o d}^{S t, f}$.

2. Each $M \in{ }_{A} \mathbf{M o d}^{S t}$ is a topological A-module and a Fréchet space.

3. All morphisms in ${ }_{A} \mathbf{M o d}^{S t}$, i.e., A-linear maps, are continuous and have a closed image, especially every f.g. submodule of a Stein module is closed.

4. A submodule $N$ of $M \in{ }_{A} \mathbf{M o d}^{S t}$ resp. its factor module $M / N$ belong to ${ }_{A} \mathbf{M o d}^{S t}$ if and only if $N$ is closed. Hence ${ }_{A} \mathbf{M o d}^{S t}$ is closed under taking images, kernels, cokernels and f.g. submodules. In particular, ${ }_{A} \mathbf{M o d}^{S t}$ contains all finitely presented A-modules, up to isomorphism of the form

$$
A^{1 \times q} / A^{1 \times k} R=\operatorname{cok}\left(A^{1 \times k} \stackrel{\circ R}{\longrightarrow} A^{1 \times q}\right), R \in A^{k \times q},
$$


and hence the full subcategory ${ }_{A} \operatorname{Mod}^{f p} \subset{ }_{A}$ Mod of all finitely presented $A$-modules is contained in ${ }_{A} \mathbf{M o d}^{S t}$.

5. If $\mathfrak{a}$ is a closed ideal of $A$ then $A / \mathfrak{a}$ is also a Stein algebra.

6. The sum $U_{1}+U_{2}$ of two closed submodules of a Stein module $M$ is closed

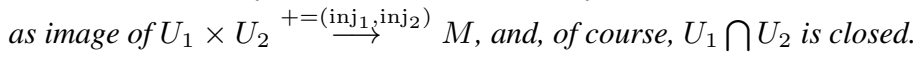

7. The family of functors

$\mathcal{O}_{z} \otimes_{A}(-):{ }_{A} \operatorname{Mod}^{S t} \rightarrow_{\mathcal{O}_{z}} \operatorname{Mod}^{f}, M \mapsto M_{z}:=\mathcal{O}_{z} \otimes_{A} M, z \in Z$,

is faithfully flat in the sense that a sequence

$$
\begin{aligned}
& M_{1} \stackrel{\phi_{1}}{\longrightarrow} M_{2} \stackrel{\phi_{2}}{\longrightarrow} M_{3} \text { in }{ }_{A} \mathbf{M o d}^{S t} \text { is exact if and only if all sequences } \\
& \mathcal{O}_{z} \otimes_{A} M_{1} \stackrel{\mathcal{O}_{z} \otimes \phi_{1}}{\longrightarrow} \mathcal{O}_{z} \otimes_{A} M_{2} \stackrel{\mathcal{O}_{z} \otimes \phi_{2}}{\longrightarrow} \mathcal{O}_{z} \otimes_{A} M_{3}, z \in Z, \text { are exact. }
\end{aligned}
$$

In particular, $M=0$ if and only if $M_{z}=0$ for all $z \in Z$.

8. If $N$ is any submodule of the Stein module $M$ its closure $\bar{N}$ is the unique closed and hence Stein submodule $U$ of $M$ such that

$$
U_{z}=\sum_{n \in N} \mathcal{O}_{z} n_{z} \subseteq M_{z} \text { for all } z \in Z,\left(n_{z}=1 \otimes n\right) .
$$

9. There are the bijections

$Z \cong \operatorname{Al}_{\mathbb{C}}(A, \mathbb{C}) \cong\{\mathfrak{m}$ closed maximal ideal of $A\}, z \leftrightarrow \rho_{z} \leftrightarrow \mathfrak{m}(z)=\operatorname{ker}\left(\rho_{z}\right)$,

where $\operatorname{Al}_{\mathbb{C}}(A, B)$ denotes the set of $\mathbb{C}$-algebra homomorphisms. A maximal ideal is closed if and only if it is f.g.

The assertion of item 7. follows from the isomorphisms (62) and the fact that the exactness of a sequence of abelian sheaves is equivalent with the exactness of all induced fibre sequences.

For $Z=\mathbb{C}^{n}$ one obtains

$$
\begin{array}{cccc}
A=\mathcal{O}\left(\mathbb{C}_{s}^{n}\right) & \subset & \mathcal{O}_{z}=\mathbb{C}<s-z>, \\
\mathfrak{m}(z)=\sum_{i=1}^{n} A\left(s_{i}-z_{i}\right) & \subset & \mathfrak{m}_{z}=\sum_{i=1}^{n} \mathbb{C}<s-z>\left(s_{i}-z_{i}\right)
\end{array}, z \in Z .
$$

In the sequel we only use the data and properties of $A$ and ${ }_{A} \operatorname{Mod}^{S t}$ from the preceding result. We study the properties of the dual module $A^{\prime}$, in particular, from Section 3, the $\mathcal{O}\left(\mathbb{C}_{s}^{n}\right)$-signal module $\mathcal{O}\left(\mathbb{C}_{s}^{n}\right)^{\prime} \cong \mathbb{C}<x>\cong \mathcal{O}\left(\mathbb{C}_{x}^{n} ; \exp \right)$. If $M \in{ }_{A} \mathbf{M o d}^{S t}$ let again $M^{\prime}$ denote the topological dual of $M$ (with its canonical Fréchet topology), i.e., the space of continuous $\mathbb{C}$-linear functions from $M$ to $\mathbb{C}$. Since $M$ is a topological $A$-module the dual $M^{\prime}$ is an $A$-module with the action

$$
(f \circ \varphi)(x)=\varphi(f x), f \in A, x \in M, \varphi \in M^{\prime} .
$$

In particular, $A^{\prime}$ is an $A$-module and therefore ${ }_{A} A^{\prime}$-behaviors are defined according to Section 2. We obtain the two functors

$$
(-)^{\prime}, \operatorname{Hom}_{A}\left(-, A^{\prime}\right):{ }_{A} \operatorname{Mod}^{S t} \rightarrow_{A} \operatorname{Mod} .
$$

We also consider the canonical $\mathbb{C}$-bilinear form

$$
\langle-,-\rangle_{M}: M \times M^{\prime} \rightarrow \mathbb{C},\langle m, \varphi\rangle_{M}:=\varphi(m) .
$$

This form is non-degenerate, i.e. here, $\langle m,-\rangle_{M}=0$ implies $m=0$ since $M$ is a Hausdorff locally convex space. The form induces the weak locally convex 
topologies on $M$ and $M^{\prime}$ [5, §II.6,2]. For each subspace $U$ of $M$ there is its polar space

$$
\begin{gathered}
U^{o}:=\left\{\varphi \in M^{\prime} ;\langle U, \varphi\rangle_{M}=\varphi(U)=0\right\} \cong(M / U)^{\prime}, \\
\varphi \leftrightarrow \varphi_{\text {ind }}=(m+U \mapsto \varphi(m))
\end{gathered}
$$

where, of course, $M / U$ carries the quotient topology. Likewise each subspace $\mathcal{B} \subseteq M^{\prime}$ gives rise to its polar subspace $\mathcal{B}^{o} \subseteq M$. The closed subspaces in the weak and the canonical locally convex topology of $M$ coincide and $U^{o o}=\bar{U}$ for any subspace $U$ of $M$, i.e., the bipolar space of $U$ coincides with its closure [5, Cor.2 and 3 of Th. II.6.1 (bipolar theorem)].

As in (49) there is also the functorial monomorphism

$$
\epsilon_{M}: M^{\prime} \rightarrow \operatorname{Hom}_{A}\left(M, A^{\prime}\right), \varphi \mapsto \phi, \phi(m)(f)=\varphi(f m), M \in_{A} \operatorname{Mod}^{S t} .
$$

In the last item of the following theorem we use that for each $z \in Z$ the ring $\mathcal{O}_{z}$ is an analytic local algebra with its sequence topology and that $\mathcal{O}_{z}^{\prime}$ is an injective cogenerator over $\mathcal{O}_{z}$ by Th. 4.3. Via $\operatorname{can}_{z}: A \rightarrow \mathcal{O}_{z}$ these $\mathcal{O}_{Z}^{\prime}$ are also $A$ modules and so is their coproduct or external direct sum

$$
W_{\ell}:=\coprod_{z \in Z} \mathcal{O}_{z}^{\prime}, \text { especially } W_{\ell} \cong \coprod_{z \in Z} \mathcal{O}\left(\mathbb{C}_{x}^{n} ; \text { infexp }\right) e^{z \bullet x} \subset \mathcal{O}\left(\mathbb{C}_{x}^{n} ; \exp \right)^{(Z)}
$$

for $Z=\mathbb{C}^{n}$.

Theorem 6.2. (Elimination and duality for ${ }_{A} A^{\prime}$-behaviors) Data from Result 6.1.

1. For $q \in \mathbb{N}$ there is the duality

$$
\begin{array}{cccc}
\left\{U \in \mathbb{P}\left(A^{1 \times q}\right) ; U \text { closed }\right\} & \cong & \left.\mathcal{B} \subseteq A^{\prime q} ; \mathcal{B} \text { generalized }{ }_{A} A^{\prime} \text {-behavior }\right\} \\
U=\mathcal{B}^{\perp} & \leftrightarrow & \mathcal{B}=U^{\perp}
\end{array}
$$

This is even a lattice duality, i.e., if $U_{i}, i=1,2$, are closed with associated behavior $\mathcal{B}_{i}=U_{i}^{\perp}$ then

$$
\mathcal{B}_{1} \bigcap \mathcal{B}_{2}=\left(U_{1}+U_{2}\right)^{\perp} \text { and } \mathcal{B}_{1}+\mathcal{B}_{2}=\left(U_{1} \bigcap U_{2}\right)^{\perp} .
$$

2. If $M$ is a f.g. Stein module then $\epsilon_{M}$ is an isomorphism. It is open, but unlikely that $\epsilon_{M}$ is an isomorphism for arbitrary Stein modules.

3. The functor $(-)^{\prime}:{ }_{A} \operatorname{Mod}^{S t} \rightarrow_{A} \operatorname{Mod}$ is faithfully exact, i.e., a sequence

$$
\begin{gathered}
M_{1} \stackrel{\phi_{1}}{\longrightarrow} M_{2} \stackrel{\phi_{2}}{\longrightarrow} M_{3} \text { in }{ }_{A} \mathbf{M o d}^{S t} \text { is exact if and only if } \\
M_{1}^{\prime} \stackrel{\phi_{1}^{\prime}}{\longleftarrow} M_{2}^{\prime} \stackrel{\phi_{2}^{\prime}}{\longleftarrow} M_{3}^{\prime} \text { is exact. }
\end{gathered}
$$

With item 2. this implies that the functor $\operatorname{Hom}_{A}\left(-, A^{\prime}\right)$ is faithfully exact on the category ${ }_{A} \operatorname{Mod}^{S t, f}$ off.g. Stein modules, i.e., if

$$
\begin{aligned}
& U_{i}=\overline{U_{i}} \subseteq A^{1 \times q_{i}}, i=1,2,3, P_{1} \in A^{q_{1} \times q_{2}}, P_{2} \in A^{q_{2} \times q_{3}} \text { with } \\
& U_{1} P_{1} \subseteq U_{2}, U_{2} P_{2} \subseteq U_{3} \text { and generalized }{ }_{A} A^{\prime} \text {-behaviors } \mathcal{B}_{i}:=U_{i}^{\perp} \text { then } \\
& A^{1 \times q_{1}} / U_{1} \stackrel{\left(\circ P_{1}\right)_{\text {ind }}}{\longrightarrow} A^{1 \times q_{2}} / U_{2} \stackrel{\left(\circ P_{2}\right)_{\text {ind }}}{\longrightarrow} A^{1 \times q_{3}} / U_{3} \text { is exact if and only if } \\
& \mathcal{B}_{1} \stackrel{P_{1} \circ}{\longleftarrow} \mathcal{B}_{2} \stackrel{P_{2} \circ}{\longleftarrow} \mathcal{B}_{3} \text { is exact. }
\end{aligned}
$$

We say shortly that ${ }_{A} A^{\prime}$ is an injective cogenerator for the category of f.g. Stein modules [6, Cor. and Def. 2.8] although $W$ itself is not a Stein module. In particular, ${ }_{A} A^{\prime}$ satisfies the weak Baer condition of Lemma 2.4 
4. Elimination and the fundamental principle hold for generalized ${ }_{A} A^{\prime}$-behaviors. More precisely, if $P_{1} \in A^{q_{1} \times q_{2}}$ and $U_{2}$ is a closed $A$-submodule of $A^{1 \times q_{2}}$ then $P_{1} \circ U_{2}^{\perp}=\left(\left(\circ P_{1}\right)^{-1}\left(U_{2}\right)\right)^{\perp}$. If $A$ is coherent then the image of ${ }_{A} A^{\prime}$ behaviors is again such. If $\operatorname{ker}\left(\circ P_{1}\right)=0$ then $P_{1} \circ\left(A^{\prime}\right)^{q_{2}}=\left(A^{\prime}\right)^{q_{1}}$, especially $P_{1} \circ A^{\prime}=A^{\prime}$ if $P$ is a non-zero divisor of $A$. This is the case if $A$ is a domain, for instance $\mathcal{O}\left(\mathbb{C}_{s}^{n}\right)$, and $P_{1}$ is nonzero and signifies that $A^{\prime}$ is a divisible $A$-module.

5. The contravariant functor

$$
\operatorname{Hom}_{A}\left(-, W_{\ell}\right):{ }_{A} \operatorname{Mod}^{S t, f} \rightarrow_{A} \operatorname{Mod}, W_{\ell}:=\coprod_{z \in Z} \mathcal{O}_{z}^{\prime},
$$

is also faithfully exact, i.e., preserves and reflects exact sequences. Therefore item 4 also holds for ${ }_{A} W_{\ell}$-behaviors.

Proof. 1. If $U \subseteq A^{1 \times q}$ resp. $\mathcal{B} \subseteq A^{\prime q}$ are arbitrary $A$-submodules then $U^{o}=U^{\perp}$ and $\mathcal{B}^{o}=\mathcal{B}^{\perp}$ as was shown in [6, Th. 2.23]. This and the mentioned bipolar theorem imply

$$
\bar{U}=U^{o o}=U^{\perp \perp}, \text { hence } U=U^{\perp \perp} \Longleftrightarrow U \text { is closed. }
$$

The duality now follows from (11). The equality $\mathcal{B}_{1} \cap \mathcal{B}_{2}=\left(U_{1}+U_{2}\right)^{\perp}$ is obvious; from Result 6.1 we know that $U_{1}+U_{2}$ is closed. The other equality is shown below.

2. We choose any $A$-epimorphism $\nu: A^{1 \times q} \rightarrow M$ and use Result 6.1: The epimorphism $\nu$ is continuous and open by the open mapping theorem, hence $U:=$ $\operatorname{ker}(\nu)$ is closed, $A^{1 \times q} / U \in_{A} \operatorname{Mod}^{S t}$ and $A^{1 \times q} / U \cong M$ is a topological isomorphism. Hence we may assume $A^{1 \times q} / U=M$ for simplicity. We identify $A^{\prime q}=\left(A^{1 \times q}\right)^{\prime}$ and infer the isomorphisms

$$
M^{\prime}=\left(A^{1 \times q} / U\right)^{\prime} \underset{(69)}{\cong} U^{o} \underset{(75)}{=} U^{\perp} \underset{(12)}{\cong} \operatorname{Hom}_{A}\left(A^{1 \times q} / U, A^{\prime}\right)=\operatorname{Hom}_{A}\left(M, A^{\prime}\right) .
$$

It is easily checked that the isomorphism from left to right coincides with $\epsilon_{M}$. 3. The proof proceeds as that of Theorem 4.3.

Exactness: The first exact sequence in (72) induces the exact sequences

$$
M_{1} \stackrel{\phi_{1}}{\longrightarrow} M_{2} \stackrel{\phi_{2, \text { ind }}}{\longrightarrow} N:=\operatorname{im}\left(\phi_{2}\right) \rightarrow 0 \text { and } 0 \rightarrow N \stackrel{\text { inj }}{\longrightarrow} M_{3} \text { with } \phi_{2}=\operatorname{inj} \phi_{2, \text { ind }} \text {. }
$$

Then $N$ is closed and contained in ${ }_{A} \operatorname{Mod}{ }^{S t}$. The spaces $M_{2}$ and $N$ are Fréchet spaces. The map $\phi_{2, \text { ind }}$ is continuous and surjective and thus open by the open mapping theorem. The Hahn-Banach theorem implies the surjection inj' $: M_{3}^{\prime} \rightarrow$ $N^{\prime}$. There result the exact sequences

$$
M_{1}^{\prime} \stackrel{\phi_{1}^{\prime}}{\longleftarrow} M_{2}^{\prime} \stackrel{\phi_{2, \text { ind }}^{\prime}}{\longleftarrow} N^{\prime} \leftarrow 0 \text { and } 0 \leftarrow N^{\prime} \stackrel{\text { inj }}{\longleftarrow} M_{3}^{\prime} \text { with } \phi_{2}^{\prime}=\phi_{2, \text { ind }}^{\prime} \text { inj }{ }^{\prime}
$$

which imply the asserted exact sequence $M_{1}^{\prime} \stackrel{\phi_{1}^{\prime}}{\longleftarrow} M_{2}^{\prime} \stackrel{\phi_{2}^{\prime}}{\longleftarrow} M_{3}^{\prime}$.

Faithfulness: (i) Since any Stein module $M$ is a Fréchet space $M=0$ if and only if $M^{\prime}=0$.

(ii) The functor $(-)^{\prime}$ is faithful, i.e., $\phi: M_{1} \rightarrow M_{2}$ is zero if and only if $\phi^{\prime}=0$ : Assume $\phi^{\prime}=0$. Consider the standard factorization

$$
\phi=\operatorname{inj} \phi_{\text {ind }}: M_{1} \stackrel{\phi_{\text {ind }}}{\longrightarrow} N:=\operatorname{im}(\phi) \stackrel{\text { inj }}{\longrightarrow} M_{2} .
$$

Exactness implies the factorization $0=\phi^{\prime}=\phi_{\text {ind }}^{\prime}$ inj $^{\prime}$ with a monomorphism $\phi_{\text {ind }}^{\prime}$ and epimorphism inj', hence

$$
\text { inj }^{\prime}=0 \Longrightarrow N^{\prime}=0 \underset{(i)}{\Longrightarrow} N=0 \Longrightarrow \phi=0 .
$$


(iii) If $\phi^{\prime}$ is an epimorphism then $\phi$ is a monomorphism: The exact sequence

$0 \rightarrow \operatorname{ker}(\phi) \rightarrow M_{1} \stackrel{\phi}{\rightarrow} M_{2}$ implies the exact sequence $0 \leftarrow \operatorname{ker}(\phi)^{\prime} \leftarrow M_{1} \stackrel{\phi^{\prime}}{\leftarrow} M_{2}$.

If $\phi^{\prime}$ is an epimorphism then $\operatorname{ker}(\phi)^{\prime}=0$ and therefore, by (i), $\operatorname{ker}(\phi)=0$ and the injectivity of $\phi$.

(iv) The second exact sequence in (72) implies $0=\phi_{1}^{\prime} \phi_{2}^{\prime}=\left(\phi_{2} \phi_{1}\right)^{\prime}=0$. From (ii) we infer $\phi_{2} \phi_{1}=0$ and thus that the first sequence in (72) is a complex. It implies the first exact sequence in (76), factorizations $\phi_{2}=\psi \phi_{2, \text { ind }}$ and $\phi_{2}^{\prime}=$ $\phi_{2, \text { ind }}^{\prime} \psi^{\prime}$ and the exact sequence

$$
\begin{gathered}
M_{1}^{\prime} \stackrel{\phi_{1}^{\prime}}{\longleftarrow} M_{2}^{\prime} \stackrel{\phi_{2, \text { ind }}^{\prime}}{\longleftarrow} N^{\prime} \leftarrow 0, \text { hence } \operatorname{ker}\left(\phi_{1}^{\prime}\right)=\operatorname{im}\left(\phi_{2, \text { ind }}^{\prime}\right)=\phi_{2, \text { ind }}^{\prime}\left(N^{\prime}\right) \text { and } \\
\operatorname{ker}\left(\phi_{1}^{\prime}\right)=\operatorname{im}\left(\phi_{2}^{\prime}\right)=\operatorname{im}\left(\phi_{2, \text { ind }}^{\prime} \psi^{\prime}\right)=\phi_{2, \text { ind }}^{\prime}\left(\operatorname{im}\left(\psi^{\prime}\right)\right) .
\end{gathered}
$$

Since $\phi_{2, \text { ind }}^{\prime}$ is a monomorphism we infer $N^{\prime}=\operatorname{im}\left(\psi^{\prime}\right)$, the surjectivity of $\psi^{\prime}$ and, by (iii), the injectivity of $\psi$. This implies

$$
\operatorname{im}\left(\phi_{1}\right)=\operatorname{ker}\left(\phi_{2, \text { ind }}\right)=\operatorname{ker}\left(\psi \phi_{2, \text { ind }}\right)=\operatorname{ker}\left(\phi_{2}\right)
$$

i.e., the exactness of the first sequence in (72).

If $\mathfrak{a}$ is a f.g. ideal with the inclusion inj : $\mathfrak{a} \rightarrow A$ then $\mathfrak{a} \in \in_{A} \operatorname{Mod}^{S t, f}$. The surjectivity of $\operatorname{Hom}\left(\mathrm{inj}, A^{\prime}\right): A^{\prime}=\operatorname{Hom}_{A}\left(A, A^{\prime}\right) \rightarrow \operatorname{Hom}_{A}\left(\mathfrak{a}, A^{\prime}\right)$ furnishes the Baer condition for $\mathfrak{a}$.

4. We use the notations from (15) and (16) with $W=A^{\prime}$. Since $U_{2} \subseteq A^{1 \times q_{2}}$ is closed and $\circ P: A^{1 \times q_{1}} \rightarrow A^{1 \times q_{2}}$ is $A$-linear and hence continuous the inverse image $(\circ P)^{-1}\left(U_{2}\right)$ is closed in $A^{1 \times q_{1}}$. Therefore

$$
\phi:=(\circ P)_{\text {ind }}: A^{1 \times q_{1}} /(\circ P)^{-1}\left(U_{2}\right) \rightarrow A^{1 \times q_{2}} / U_{2}
$$

is a monomorphism between f.g. Stein modules and by item 3. the map $\operatorname{Hom}\left(\phi, A^{\prime}\right)$ is surjective. With (16) we infer $P \circ U_{2}^{\perp}=\left((\circ P)^{-1}\left(U_{2}\right)\right)^{\perp}$. Consider, in particular, closed submodules

$$
\begin{gathered}
U_{i} \subseteq A^{1 \times q}, i=1,2, \text { the closed module } V:=U_{1} \times U_{2} \subseteq A^{1 \times 2 q}=A^{1 \times q} \times A^{1 \times q} \text { with } \\
V^{\perp}=U_{1}^{\perp} \times U_{2}^{\perp} \subseteq A^{\prime 2 q} \text { and } P=\left(\operatorname{id}_{q}, \operatorname{id}_{q}\right) \in A^{q \times 2 q} \text {. Then }(\circ P)^{-1}(V)=U_{1} \bigcap U_{2} \text { and } \\
\left(U_{1} \bigcap U_{2}\right)^{\perp}=\left((\circ P)^{-1}(V)\right)^{\perp}=\left(\operatorname{id}_{q}, \operatorname{id}_{q}\right) \circ\left(U_{1}^{\perp} \times U_{2}^{\perp}\right)=U_{1}^{\perp}+U_{2}^{\perp} .
\end{gathered}
$$

This proves the last assertion of item 1 .

5. For $M \in \in_{A} \operatorname{Mod}^{S t}$ we have

$M_{z}=\mathcal{O}_{z} \otimes_{A} M$, hence $\operatorname{Hom}_{A}\left(M, \mathcal{O}_{z}^{\prime}\right) \cong \operatorname{Hom}_{\mathcal{O}_{z}}\left(M_{z}, \mathcal{O}_{z}^{\prime}\right)$ and, if $M$ is f.g., also

$$
\operatorname{Hom}_{A}\left(M, \coprod_{z \in Z} \mathcal{O}_{z}^{\prime}\right)=\coprod_{z \in Z} \operatorname{Hom}_{A}\left(M, \mathcal{O}_{z}^{\prime}\right) \cong \coprod_{z \in Z} \operatorname{Hom}_{\mathcal{O}_{z}}\left(M_{z}, \mathcal{O}_{z}^{\prime}\right) \text {. }
$$

Consider a sequence

$\mathfrak{M}: M_{1} \stackrel{\phi_{1}}{\longrightarrow} M_{2} \stackrel{\phi_{2}}{\longrightarrow} M_{3}$ in ${ }_{A} \mathbf{M o d}^{S t, f}$ and $\mathfrak{M}_{z}:=\mathcal{O}_{z} \otimes_{A} \mathfrak{M}$ in $\mathcal{O}_{z} \mathbf{M o d}^{f}$. Then

$\mathfrak{M}$ exact $\underset{\text { Res. } 6.1,(7)}{\Longleftrightarrow} \forall z \in Z: \mathfrak{M}_{z}$ exact $\underset{\mathcal{O}_{z}}{\Longleftrightarrow} \underset{\mathcal{O}_{z}^{\prime} \text { inj. cog. }}{\Longleftrightarrow}$

$\forall z \in Z: \operatorname{Hom}_{\mathcal{O}_{z}}\left(\mathfrak{M}_{z}, \mathcal{O}_{z}^{\prime}\right)$ exact $\underset{(77)}{\Longleftrightarrow} \operatorname{Hom}_{A}\left(\mathfrak{M}, \coprod_{z \in Z} \mathcal{O}_{z}^{\prime}\right)$ exact. 
Corollary 6.3. The duality and elimination statements of the preceding theorem hold for the $\mathcal{O}\left(\mathbb{C}_{s}^{n}\right)$-signal modules (compare Section 3)

$$
\begin{gathered}
\mathcal{O}\left(\mathbb{C}_{s}^{n}\right)^{\prime} \cong \mathbb{C}<x>\cong \mathcal{O}\left(\mathbb{C}_{x}^{n} ; \exp \right) \text { and } W_{\ell}:=\coprod_{z \in \mathbb{C}^{n}} \mathcal{O}_{z}^{\prime} \text { with } \\
\mathcal{O}_{z}=\mathbb{C}\left\langle s-z>\text { and } \mathcal{O}_{z}^{\prime} \cong W_{z}=\mathcal{O}\left(\mathbb{C}_{x}^{n} ; \text { infexp }\right) e^{z \bullet x} .\right.
\end{gathered}
$$

For $n>1$ it is not known, but unlikely that $\mathcal{O}\left(\mathbb{C}_{s}^{n}\right)$ is coherent (communication from $O$. Forster). Therefore it is not clear whether the image of a ${ }_{\mathcal{O}\left(\mathbb{C}_{s}^{n}\right)} \mathcal{O}\left(\mathbb{C}_{s}^{n}\right)^{\prime}$ behavior is again such. For $n=1$ every closed and especially every f.g. ideal of $\mathcal{O}\left(\mathbb{C}_{s}\right)$ is cyclic. Thus this ring is a Bézout domain and especially coherent. In particular, images of $\mathcal{O}\left(\mathbb{C}_{s}\right) \mathcal{O}\left(\mathbb{C}_{s}\right)^{\prime}$-behaviors are again such.

It is presently not known whether the sum $\sum_{z \in \mathbb{C}^{n}} W_{z}$ is direct, i.e., whether

$$
\begin{gathered}
\sum_{z \in \mathbb{C}^{n}} \mathcal{O}\left(\mathbb{C}_{x}^{n} ; \text { infexp }\right) e^{z \bullet x}=\bigoplus_{z \in \mathbb{C}^{n}} \mathcal{O}\left(\mathbb{C}_{x}^{n} ; \text { infexp }\right) e^{z \bullet x} \subset \mathcal{O}\left(\mathbb{C}_{x}^{n} ; \exp \right) \text { or } \\
\coprod_{z \in \mathbb{C}^{n}} \mathcal{O}_{z}^{\prime} \cong \sum_{z \in \mathbb{C}^{n}} \mathcal{O}\left(\mathbb{C}_{x}^{n} ; \text { infexp }\right) e^{z \bullet x} .
\end{gathered}
$$

Corollary 6.4. If $\mathfrak{a}$ is a closed ideal of $A$ then Th. 6.2 is applicable to the factor Stein algebra $B:=A / \mathfrak{a}$. A Stein B-module is the same as a Stein A-module $N$ with $\mathfrak{a} N=0$ and scalar multiplication $(f+\mathfrak{a}) x=f x, f \in A, x \in N$. Then

$$
B^{\prime}=(A / \mathfrak{a})^{\prime} \cong\left\{w \in A^{\prime} ; w(\mathfrak{a})=0\right\}=\left\{w \in A^{\prime} ; \mathfrak{a} \circ w=0\right\}=\mathfrak{a}^{\perp} .
$$

The corresponding results can all be expressed by Stein modules over A. For elimination consider $P \in A^{q_{1} \times q_{2}}$ and $U_{2}=\overline{U_{2}} \subseteq A^{1 \times q_{2}}$. Then $U_{2}+\mathfrak{a} A^{1 \times q_{2}}$ is also closed and

$$
\begin{gathered}
\left(\mathfrak{a} A^{1 \times q_{2}}\right)^{\perp}=\left(\mathfrak{a}^{\perp}\right)^{q_{2}} \subseteq\left(A^{\prime}\right)^{q_{2}},\left(U_{2}+\mathfrak{a} A^{1 \times q_{2}}\right)^{\perp}=U_{2}^{\perp} \bigcap\left(\mathfrak{a}^{\perp}\right)^{q_{2}} \text { and } \\
P \circ\left(U_{2}^{\perp} \bigcap\left(\mathfrak{a}^{\perp}\right)^{q_{2}}\right)=U_{1}^{\perp} \subseteq\left(\mathfrak{a}^{\perp}\right)^{q_{1}} \text { with } U_{1}:=(\circ P)^{-1}\left(U_{2}+\mathfrak{a} A^{1 \times q_{2}}\right) \\
\text { and } \mathfrak{a} A^{1 \times q_{1}} \subseteq U_{1} \subseteq A^{1 \times q_{1}}
\end{gathered}
$$

For $U_{2}:=0$ and $U_{2}^{\perp}=\left(A^{\prime}\right)^{q_{2}}$ the fundamental principle for $\mathfrak{a}^{\perp}$ gets the form

$$
\begin{gathered}
U_{1}=(\circ P)^{-1}\left(\mathfrak{a} A^{1 \times q_{2}}\right)=\left\{x \in A^{1 \times q_{1}} ; x P \in \mathfrak{a} A^{1 \times q_{2}}\right\} \text { and } \\
P \circ\left(\mathfrak{a}^{\perp}\right)^{q_{2}}=U_{1}^{\perp} \subseteq\left(\mathfrak{a}^{\perp}\right)^{q_{1}},
\end{gathered}
$$

i.e., an inhomogeneous system $P \circ y=u \in\left(\mathfrak{a}^{\perp}\right)^{q_{1}}$ has a solution $y \in\left(\mathfrak{a}^{\perp}\right)^{q_{2}}$ if and only $u$ satisfies the compatibility conditions $x \circ u=0$ for all $x \in U_{1}$.

These considerations are especially applicable to closed and f.g. ideals of $\mathcal{O}\left(\mathbb{C}_{s}^{n}\right)$.

The following theorem is just a special case of the preceding corollary, but important.

Theorem 6.5. (Elimination and fundamental principle for periodic functions) With the notations of the preceding corollary let

$$
\begin{gathered}
A:=\mathcal{O}\left(\mathbb{C}_{s}^{n}\right), W:=\mathcal{O}\left(\mathbb{C}_{x}^{n} ; \exp \right) \cong A^{\prime} \\
G:=\oplus_{j=1}^{m} \mathbb{Z} y^{(j)} \subseteq \mathbb{C}^{n} \text { any f.g. subgroup and } \mathfrak{a}:=\sum_{j=1}^{m} A\left(e^{y^{(j)} \bullet s}-1\right) .
\end{gathered}
$$

1. Then $W_{G, p e r}:=\mathfrak{a}^{\perp}=\{w \in W ; \forall y \in G: w(x+y)=w(x)\}$ is the $A$ submodule of $W$ of all entire $G$-periodic functions of at most exponential growth. 
2. Elimination and the fundamental principle hold for the $\mathcal{O}\left(\mathbb{C}_{s}^{n}\right) / \mathfrak{a}$-module $W_{G, p e r}$, i.e., the equations (79) and (80) hold for this module. In particular, an inhomogeneous linear system $P \circ y=u$ with given $G$-periodic $u$ has a $G$-periodic solution $y$ if and only if $x \circ u=0$ for all $x \in U_{1}$.

Compare [23] for a different treatment of elimination for periodic behaviors in other signal spaces.

Proof. Recall that $e^{y \bullet s}, y \bullet s=y_{1} s_{1}+\cdots+y_{n} s_{n}$, acts on $W$ by translation, i.e.,

$\left(e^{y \bullet s} \circ w\right)(x)=w(x+y)$, hence $\left(\left(e^{y^{(j)} \bullet s}-1\right) \circ w\right)(x)=w\left(x+y^{(j)}\right)-w(x)$.

Hence $W_{G, p e r}=\mathfrak{a}^{\perp}=\left\{w \in W ; \forall j=1, \cdots, m: w(x)=w\left(x+y^{(j)}\right)\right\}=$ $\left\{w \in \mathcal{O}\left(\mathbb{C}_{x}^{n} ; \exp \right) ; \forall y \in G: w(x)=w(x+y)\right\}=\{w \in W G$-periodic $\}$.

Examples 6.6. 1 . In the preceding theorem consider the simple special case $n=$ 1. For a nonzero entire function $f$ it is known that the group of periods is

$$
\{y \in \mathbb{C} ; \forall z \in \mathbb{C}: f(z)=f(z+y)\}=\left\{\begin{array}{l}
\{0\} \text { or } \\
\mathbb{Z} y^{(1)}, y^{(1)} \neq 0, \text { or } \\
\mathbb{Z} y^{(1)} \oplus \mathbb{Z} y^{(2)}, y^{(j)} \neq 0, \frac{y^{(1)}}{y^{(2)}} \notin \mathbb{R}
\end{array} .\right.
$$

Since $\mathbb{C} /\left(\mathbb{Z} y^{(1)} \oplus \mathbb{Z} y^{(2)}\right) \cong(\mathbb{R} / \mathbb{Z})^{2}$ is compact an entire function $f$ with group of periods $\mathbb{Z} y^{(1)} \oplus \mathbb{Z} y^{(2)}$ is constant. Therefore for $n=1$ only groups $G=\mathbb{Z} y$ are interesting in Theorem 6.5.

2. Consider the case $n=1$ and $G=\mathbb{Z}$, hence

$A=\mathcal{O}\left(\mathbb{C}_{s}\right), \mathfrak{a}=A\left(e^{s}-1\right)$ and $W_{\mathbb{Z}, p e r}=\left\{w \in \mathcal{O}\left(\mathbb{C}_{x} ; \exp \right) ; w(x)=w(x+1)\right\}$

The map $e^{2 \pi i x}: \mathbb{C}_{x} \rightarrow \mathbb{C}_{x}^{*}:=\mathbb{C}_{x} \backslash 0$ is surjective and locally biholomorphic, hence

$$
\begin{gathered}
\mathcal{O}\left(\mathbb{C}_{x}^{*}\right)=\left\{\widetilde{w}=\sum_{k \in \mathbb{Z}} \widetilde{w}_{k} x^{k}\right\} \cong \mathcal{O}\left(\mathbb{C}_{x}\right)_{p e r}:=\left\{w \in \mathcal{O}\left(\mathbb{C}_{x}\right) ; w(x+1)=w(x) .\right\} \\
\widetilde{w}(x) \leftrightarrow w(x)=\widetilde{w}\left(e^{2 \pi i x}\right)=\sum_{k \in \mathbb{Z}} \widetilde{w}_{k} e^{2 \pi i k x} .
\end{gathered}
$$

We discuss the fundamental principle for $W_{\mathbb{Z}, p e r}$ in the simplest case: Let

$$
e:=e^{s}-1, \mathfrak{a}=A e, \text { and } P \in A=\mathcal{O}\left(\mathbb{C}_{s}\right), P \neq 0,
$$

$$
\text { with zero sets } V_{\mathbb{C}}(P):=\{z \in \mathbb{C} ; P(z)=0\} \text { and } V_{\mathbb{C}}(e)=2 \pi i \mathbb{Z} \text {. }
$$

The ring $A$ is a Bézout domain, i.e., each f.g. ideal is principal, and therefore admits greatest common divisors (gcd) and least common multiples $(\mathrm{lcm})$. In particular, let

$$
\begin{gathered}
A e+A P=A d \Longrightarrow d=\operatorname{gcd}(e, P), \frac{e P}{d}=\operatorname{lcm}(e, P) \Longrightarrow \\
\mathfrak{b}:=\{f \in A, f P \in \mathfrak{a}\}=A \frac{e}{d} \underset{T h .6 .5,2 .}{\Longrightarrow} \\
P \circ W_{\mathbb{Z}, p e r}=\mathfrak{b}^{\perp}=\left\{u \in W_{\mathbb{Z}, p e r} ; \frac{e}{d} \circ u=0\right\} .
\end{gathered}
$$


Hence the equation $P \circ y=u \in W_{\mathbb{Z} \text {,per }}$ has a periodic solution $y$ if and only if $\frac{e}{d} \circ u=0$. But $W=\mathcal{O}\left(\mathbb{C}_{x} ; \exp \right)$ is divisible and thus there is $v \in W$ with

$$
d \circ v=u, \text { hence }\left(\frac{e}{d} \circ u\right)(x)=(e \circ v)(x)=v(x+1)-v(x) .
$$

Hence a periodic solution $y$ exists if and only if $v$ is periodic. Notice that $v$ is not unique, but that all solutions of $d \circ v=0$ are periodic since $d$ divides $e$, so the preceding statement does not depend on the choice of $v$. The local ideals $\mathfrak{a}_{z}$ are

$$
\begin{gathered}
\mathfrak{a}_{z}=\left\{\begin{array}{ll}
\mathcal{O}_{z} & \text { if } z \notin \mathbb{Z} 2 \pi i \\
\mathfrak{m}_{z}=\mathcal{O}_{z}(s-z) & \text { if } z \in \mathbb{Z} 2 \pi i
\end{array} \Longrightarrow\right. \\
(A d)_{z}=\mathfrak{a}_{z}+\mathcal{O}_{z} P= \begin{cases}\mathfrak{m}_{z}=\mathcal{O}_{z}(s-z) & \text { if } z \in 2 \pi i \mathbb{Z} \cap V_{\mathbb{C}}(P) \\
\mathcal{O}_{z} & \text { otherwise }\end{cases}
\end{gathered}
$$

So $d$ is entire with zeros of order 1 exactly at the points $z \in 2 \pi i \mathbb{Z} \cap V_{\mathbb{C}}(P)$. Assume especially that $P$ is a nonzero polynomial. Then

$$
2 \pi i \mathbb{Z} \cap V_{\mathbb{C}}(P) \text { is finite and } d=\prod_{z \in 2 \pi i \mathbb{Z} \cap V_{\mathbb{C}}(P)}(s-z) \in \mathbb{C}[s] .
$$

The equations $P \circ y=u \in W_{\mathbb{Z}, \text { per }}$ and $d \circ v=u$ are ordinary differential equations and can be solved. The first one has a periodic solution if and only if one or all solutions $v$ of the second are periodic. This example can also be treated by means of the Bézout domain [12, Def. 2.9], [13, Th. 2.2 and 5.4]

$\mathcal{H}:=\operatorname{quot}\left(\mathbb{C}\left[s, e^{s}, e^{-s}\right]\right) \bigcap \mathcal{O}\left(\mathbb{C}_{s}\right)=\operatorname{quot}(\mathbb{C}(s))\left[e^{s}, e^{-s}\right] \bigcap \mathcal{O}\left(\mathbb{C}_{s}\right) \subset \mathcal{M}\left(\mathbb{C}_{s}\right)$

where $\mathcal{M}\left(\mathbb{C}_{s}\right)=\operatorname{quot}\left(\mathcal{O}\left(\mathbb{C}_{s}\right)\right)$ is the field of meromorphic functions.

3. Similar considerations are possible for arbitrary $n \geq 1$ and $G=\mathbb{Z}^{n}$. Then

$$
\begin{gathered}
\mathcal{O}\left(\left(\mathbb{C}_{x} \backslash\{0\}\right)^{n}\right) \cong \mathcal{O}\left(\mathbb{C}_{x}^{n}\right)_{\text {per }}:=\left\{w \in \mathcal{O}\left(\mathbb{C}_{x}^{n}\right) ; \forall g \in \mathbb{Z}^{n}: w(x)=w(x+g)\right\} \\
\widetilde{w}(x)=\sum_{\mu \in \mathbb{Z}^{n}} \widetilde{w}_{\mu} x^{\mu} \longleftrightarrow w(x)=\sum_{\mu \in \mathbb{Z}^{n}} \widetilde{w}_{\mu} e^{2 \pi i \mu \bullet x} .
\end{gathered}
$$

Remark 6.7. In general, $A^{\prime}$ is not an $A$-cogenerator (see Lemma 2.5). Indeed, let $Z$ be a Stein space which contains an infinite discrete subset, for instance $\mathbb{C}^{n}$ for $n>0$. Then [16, Bem. 3 on p.182] has a non-closed maximal ideal $\mathfrak{m}$. Assume that ${ }_{A} A^{\prime}$ is a cogenerator. Then the module $A / \mathfrak{m}$ admits a non-zero homomorphism into $A^{\prime}$ which is a monomorphism since $A / \mathfrak{m}$ is simple. Hence there is a $w \in A^{\prime}$ such that $\mathfrak{m}$ is the kernel of $A \rightarrow A^{\prime}, f \mapsto f \circ w$. Since $A$ is a topological algebra this map is continuous with respect to the canonical topology on $A$ and the weak topology on $A^{\prime}$ and hence its kernel $\mathfrak{m}$ is closed, a contradiction.

The next lemma will be used in Section 8. We use the data from (58) and Th. 6.2 , item 9. For a Stein module $M$ and $z \in Z$ there are the quotient ring, quotient module and canonical maps

$$
\begin{gathered}
A_{\mathfrak{m}(z)}:=\left\{\frac{f}{g} ; f, g \in A, g(z) \neq 0\right\} \text { and } \\
A_{\mathfrak{m}(z)} M_{\mathfrak{m}(z)}:=\left\{\frac{x}{g} ; x \in M, g \in A, g(z) \neq 0\right\}, \\
\operatorname{can~}: A_{\mathfrak{m}(z)} \rightarrow \mathcal{O}_{z}, \frac{f}{g} \mapsto g_{z}^{-1} f_{z}, \operatorname{can}: M_{\mathfrak{m}(z)} \rightarrow M_{z}, \frac{x}{g} \mapsto g_{z}^{-1} x_{z} .
\end{gathered}
$$

Recall that $g(z) \neq 0$ signifies that $g_{z} \notin \mathfrak{m}_{z}$ or that $g_{z}$ is a unit in $\mathcal{O}_{z}$. 
Lemma 6.8. Data as in (81).

1. For each $z \in Z$ the canonical map can : $M_{\mathfrak{m}(z)} \rightarrow M_{z}$ is injective and $A_{\mathfrak{m}(z)} M_{\mathfrak{m}(z)}$ is noetherian. This applies especially to $M=A$ and $A_{z}=\mathcal{O}_{z}$. In the sequel we identify

$$
M_{\mathfrak{m}(z)} \subseteq M_{z}, \frac{x}{g}=g_{z}^{-1} x_{z}, A_{\mathfrak{m}(z)} \subseteq \mathcal{O}_{z}, \frac{f}{g}=g_{z}^{-1} f_{z} .
$$

2. If $A$ is an integral domain then for all $z \in Z$ there are the injections

$$
A \stackrel{\text { inj }}{\longrightarrow} A_{\mathfrak{m}(z)} \longrightarrow \mathcal{O}_{z}, f \mapsto \frac{f}{1} \mapsto f_{z},
$$

and this signifies that the identity theorem holds in $Z$.

Proof. (i) Let $\frac{x}{g} \in M_{\mathfrak{m}(z)}$ and $g_{z}^{-1} x_{z}=0 \in M_{z}$, hence $x_{z}=0$. The ideal $\mathfrak{m}(z)$ is f.g. and so are the submodules $U:=\mathfrak{m}(z) x \subseteq A x \subseteq M$, thus $U$ and $A x$ are closed and Stein. For $z \neq y \in Z$ the maximal ideals $\mathfrak{m}(z)$ and $\mathfrak{m}(y)$ are coprime, i.e.,

$$
\begin{array}{r}
A=\mathfrak{m}(z)+\mathfrak{m}(y) \text { and } \mathcal{O}_{y}=\mathfrak{m}(z)_{y}+\mathfrak{m}(y)_{y}, \mathfrak{m}(y)_{y}=\mathfrak{m}_{y}, \text { hence (Nakayama's Lemma) } \\
\mathfrak{m}(z)_{y}=\left\{\begin{array}{l}
\mathcal{O}_{y} \text { if } y \neq z, \\
\mathfrak{m}_{z} \text { if } y=z
\end{array} \text { and thus } U_{y}=\left\{\begin{array}{l}
\mathcal{O}_{y} x=(A x)_{y} \text { if } y \neq z \\
(A x)_{z}=\mathcal{O}_{z} x_{z}=0 \text { if } y=z
\end{array} .\right.\right.
\end{array}
$$

We infer $U=\mathfrak{m}(z) x=A x$ by Result 6.2 , item 7. Thus there is

$$
\begin{gathered}
h \in \mathfrak{m}(z) \text { with } x=h x \text { or }(1-h) x=0 \text { and } 1-h \notin \mathfrak{m}(z) \Longrightarrow \\
\frac{x}{g}=\frac{(1-h) x}{(1-h) g}=\frac{0}{(1-h) g}=0 \in M_{\mathfrak{m}(z) .}
\end{gathered}
$$

(ii) A module is noetherian if and only if each submodule is f.g. or if each ascending sequence of f.g.(!) submodules is stationary. Any such sequence in $M_{\mathfrak{m}(z)}$ is of the form

$$
N_{1}=\left(U_{1}\right)_{\mathfrak{m}(z)} \subseteq N_{2}=\left(U_{2}\right)_{\mathfrak{m}(z)} \subseteq \cdots \subseteq M_{\mathfrak{m}(z)}
$$

where the $U_{i}$ are f.g. and thus closed submodules of $M$. Then the submodules $M_{i}:=U_{1}+\cdots+U_{i}$ form an ascending sequence of f.g. submodules of $M$ with $\left(M_{i}\right)_{\mathfrak{m}(z)}=N_{i}$. Since $M_{z}$ is noetherian over $\mathcal{O}_{z}$ the sequence $\left(M_{1}\right)_{z} \subseteq$ $\left(M_{2}\right)_{z} \subseteq \cdots \subseteq M_{z}$ becomes stationary, hence there is an index $i_{0}$ with

$$
\forall i \geq i_{0}:\left(M_{i} / M_{i-1}\right)_{z}=\left(M_{i}\right)_{z} /\left(M_{i-1}\right)_{z}=0 \Longrightarrow
$$$$
N_{i} / N_{i-1}=\left(M_{i}\right)_{\mathfrak{m}(z)} /\left(M_{i-1}\right)_{\mathfrak{m}(z)}=\left(M_{i} / M_{i-1}\right)_{\mathfrak{m}(z)} \underset{(i)}{=} 0 \Longrightarrow N_{i}=N_{i-1} \text {. }
$$

\section{Partial differential-difference equations}

In this section we assume subrings

$$
C:=\mathbb{C}[s] \subseteq B \subseteq A:=\mathcal{O}\left(\mathbb{C}_{s}^{n}\right) \text { and } W=\mathcal{O}\left(\mathbb{C}_{x}^{n} ; \exp \right) .
$$

All these rings are contained in the quotient field $\mathcal{M}\left(\mathbb{C}_{s}^{n}\right)=\operatorname{quot}\left(\mathcal{O}\left(\mathbb{C}_{s}^{n}\right)\right)$ of meromorphic functions [15, Satz V.2.3]. The $A$-module $W$ is also a $B$-module and 
therefore ${ }_{B} W$-behaviors are defined to which the theory of Section 2 is applicable. The main application which we have in mind is the following: Let

$$
\begin{gathered}
G:=\oplus_{j=1}^{m} \mathbb{Z} y^{(j)} \subseteq \mathbb{C}^{n} \text { be any f.g. subgroup and } \sigma_{j}:=e^{y^{(j)} \bullet s} \text {. Then } \\
\mathbb{C}\left[s, \sigma, \sigma^{-1}\right]:=\mathbb{C}\left[s_{1}, \cdots, s_{n}, \sigma_{1}, \sigma_{1}^{-1}, \cdots, \sigma_{m}, \sigma_{m}^{-1}\right]=\oplus_{y \in G} \mathbb{C}[s] e^{y \bullet s}
\end{gathered}
$$

is a Laurent polynomial algebra in the indeterminates $s_{i}$ and $\sigma_{j}$ and also the algebra of polynomial-exponential functions with frequencies $y \in G$. It acts on $W$ by partial differential-difference operators since

$s_{i} \circ w=\partial w / \partial x_{i},\left(e^{y \bullet s} \circ w\right)(x)=w(x+y), y \in G,\left(\sigma_{j} \circ w\right)(x)=w\left(x+y^{(j)}\right)$.

For $n=m=1$ resp. for $n=1, m>1$, the operators in $\mathbb{C}\left[s, \sigma, \sigma^{-1}\right]$ are called delay-differential (DD) with commensurate resp. incommensurate delays. The larger rings [3, Th. 1], [6, Lemma 4.10]

$$
B:=\operatorname{quot}\left(\mathbb{C}\left[s, \sigma, \sigma^{-1}\right]\right) \bigcap \mathcal{O}\left(\mathbb{C}_{s}^{n}\right)=\mathbb{C}(s)\left[\sigma, \sigma^{-1}\right] \bigcap \mathcal{O}\left(\mathbb{C}_{s}^{n}\right) \subseteq \mathcal{O}\left(\mathbb{C}_{s}^{n}\right) \subset \mathcal{M}\left(\mathbb{C}_{s}^{n}\right)
$$

play an important part in analysis and systems theory. We note the simple fact that

$$
D \subseteq A \text { and } B:=\operatorname{quot}(D) \bigcap A \text { implies } B=\operatorname{quot}(B) \bigcap A .
$$

The book [12] contains an in-depth study of behaviors over $B$ for $n=m=1$ where in contrast to the present paper the larger signal module of $\mathrm{C}^{\infty}$-functions is used. The papers [18] and [13] treat the case of DD-behaviors with incommensurate delays for $\mathrm{C}^{\infty}$-signals.

Faithful flatness (f.f.) plays an important in the subsequent considerations $[4, \mathrm{Ch}$. I]. Recall that a ring extension $R \subset S$ is flat resp. faithfully flat (f.f) if the functor

$$
S \otimes_{R}(-):_{R} \operatorname{Mod} \rightarrow_{S} \text { Mod }
$$

preserves resp. preserves and reflects exact sequences.

The subring $B$ of $A$ carries the induced topology from that of $A$. Since $B$ contains the polynomial algebra $\mathbb{C}[s]$ and the latter is dense in $A$ so is $B$. The free modules $B^{1 \times q} \subseteq A^{1 \times q}$ have the product topology. For a subset $U \subseteq A^{1 \times q}$ we write $\mathrm{cl}_{A}(U)$ for its closure in $A^{1 \times q}$ and likewise $\mathrm{cl}_{B}(V)$ for the closure in $B^{1 \times q}$ of $V \subseteq B^{1 \times q}$. Any subset $V \subseteq B^{1 \times q}$ gives rise to the ${ }_{B} W$-behavior

$$
\begin{gathered}
\mathcal{B}:=V^{\perp}=\operatorname{cl}_{B}(B V)^{\perp}=(A V)^{\perp}=\left(\operatorname{cl}_{A}(A V)\right)^{\perp} \text { and to } \\
V \subseteq \operatorname{cl}_{B}(B V) \subseteq \mathcal{B}^{\perp_{B}}:=B^{1 \times q} \bigcap \mathcal{B}^{\perp} .
\end{gathered}
$$

Theorem 7.1. (Duality) Let $V$ in (87) be a B-submodule, i.e., $V \in \mathbb{P}\left(B^{1 \times q}\right)$. Then

$\operatorname{cl}_{A}(V)=\operatorname{cl}_{A}(A V) \in \mathbb{P}\left(A^{1 \times q}\right)$ and $\mathcal{B}^{\perp_{B}}=V^{\perp_{B}}=\operatorname{cl}_{B}(V)=B^{1 \times q} \bigcap \operatorname{cl}_{A}(A V)$.

Thus (11) implies the duality

$$
\begin{array}{ccc}
\left\{V \in \mathbb{P}\left(B^{1 \times q}\right) ; V \text { closed in } B^{1 \times q}\right\} & \cong & \left\{\mathcal{B} \text { generalized }{ }_{B} W \text {-behavior }\right\} \\
V=\mathcal{B}^{\perp_{B}} & \longleftrightarrow & \mathcal{B}=V^{\perp}
\end{array}
$$

Proof. Let $v \in V$. Since $A$ is a topological ring the map

$$
\begin{gathered}
\varphi:=\circ v: A \rightarrow A^{1 \times q}, a \mapsto a v, \text { is continuous } \Longrightarrow \\
A v \underset{B \text { dense }}{\overline{c_{2}}} \operatorname{cl}_{A}(B) v=\varphi\left(\operatorname{cl}_{A}(B)\right) \underset{\varphi \text { continuous }}{\subseteq} \operatorname{cl}_{A}(\varphi(B))=\operatorname{cl}_{A}(B v) \subseteq \operatorname{cl}_{A}(V) \Longrightarrow \\
A V \subseteq \operatorname{cl}_{A}(V) \subseteq \operatorname{cl}_{A}(A V), \text { thus } \operatorname{cl}_{A}(V)=\operatorname{cl}_{A}(A V) .
\end{gathered}
$$


From Th. 6.2,1. we infer

$$
\begin{gathered}
V^{\perp \perp}=(A V)^{\perp \perp}=\operatorname{cl}_{A}(A V)=\operatorname{cl}_{A}(V) \Longrightarrow \\
V^{\perp \perp_{B}}=B^{1 \times q} \bigcap V^{\perp \perp}=B^{1 \times q} \bigcap \operatorname{cl}_{A}(V)=\operatorname{cl}_{B}(V),
\end{gathered}
$$

the last equality being true since $B^{1 \times q}$ carries the topology induced from that of $A^{1 \times q}$.

The usefulness of this result is limited since the equality $V=\operatorname{cl}_{B}(V)$ is hard to check. For instance, if ${ }_{B} V$ is f.g. then so is ${ }_{A} A V$ and hence $A V$ is closed and

$$
\operatorname{cl}_{B}(V)=B^{1 \times q} \bigcap A V .
$$

Corollary 7.2. 1. Each principal ideal of $B$ is closed if and only if $B=$ quot $(B) \cap A$. This explains the importance of the last equality in context with behavioral duality [12].

2. If $B \subseteq A$ is a flat extension then the following properties are equivalent:

(a) The extension $B \subseteq A$ is faithfully flat (f.f.).

(b) Each f.g. submodule $V \subseteq B^{1 \times q}$ is closed.

(c) If $V_{i}=B^{1 \times k_{i}} R_{i} \subseteq B^{1 \times q}, i=1,2$, then $V_{1} \subseteq V_{2}$, i.e.,

$\exists X \in B^{k_{1} \times k_{2}}$ with $R_{1}=X R_{2} \Longleftrightarrow V_{2}^{\perp}=\left\{w \in W^{q} ; R_{2} \circ w=0\right\} \subseteq V_{1}^{\perp}$.

Proof. 1. $\Longrightarrow$ : Each principal ideal is closed and hence, by (88), $B b_{2}=A b_{2} \bigcap B$ for all $b_{2} \in B$. For $a=\frac{b_{1}}{b_{2}} \in \operatorname{quot}(B) \bigcap A$ this implies $b_{1}=a b_{2} \in A b_{2} \bigcap B=$ $B b_{2}$ and hence $a \in B . \Longleftarrow$ : analogous.

2. $(a) \Longleftrightarrow(b)$ : The flat extension $B \subseteq A$ is f.f. if and only if $V=B^{1 \times q} \cap A V$ for all f.g. submodules $V \subseteq B^{1 \times q}$, and it suffices that this holds for f.g. ideals $V$ of $B$ [4, Prop. I.3.9]. Hence $B \subseteq A$ is f.f. if and only if

$\forall$ f.g. $V \subseteq B^{1 \times q}: V=B^{1 \times q} \bigcap A V \underset{(88)}{=} \operatorname{cl}_{B}(V) \Longleftrightarrow \forall$ f.g. $V: V$ is closed.

(b) $\Longrightarrow$ (c) follows from Theorem 7.1. (c) $\Longrightarrow(b)$ : For a f.g. $B$-submodule $V_{2} \subseteq B^{1 \times q}$ consider any f.g. module $V_{1}$ with $V_{2} \subseteq V_{1} \subseteq \operatorname{cl}_{B}\left(V_{2}\right)$. Then

$$
V_{2}^{\perp}=V_{1}^{\perp}=\operatorname{cl}_{B}\left(V_{2}\right)^{\perp} \underset{(c)}{\Longrightarrow} V_{1} \subseteq V_{2} \underset{V_{2} \subseteq V_{1}}{\Longrightarrow} V_{1}=V_{2} \Longrightarrow V_{2}=\operatorname{cl}_{B}\left(V_{2}\right) .
$$

According to (65) we define the maximal ideals

$$
\begin{gathered}
\mathfrak{m}_{A}(z):=\mathfrak{m}(z):=\{f \in A ; f(z)=0\}=\sum_{i=1}^{n} A\left(s_{i}-z_{i}\right) \supseteq \\
\mathfrak{m}_{B}(z):=B \bigcap \mathfrak{m}_{A}(z)=\{f \in B ; f(z)=0\} \supseteq \sum_{i=1}^{n} B\left(s_{i}-z_{i}\right), \\
\mathfrak{m}_{C}(z):=C \bigcap \mathfrak{m}_{A}(z):=\{f \in C ; f(z)=0\}=\sum_{i=1}^{n} C\left(s_{i}-z_{i}\right), C:=\mathbb{C}[s], \\
\text { with } C / \mathfrak{m}_{C}(z)=B / \mathfrak{m}_{B}(z)=A / \mathfrak{m}_{A}(z)=\mathbb{C} .
\end{gathered}
$$


The maximal ideals $\mathfrak{m}_{A}(z), \mathfrak{m}_{B}(z), \mathfrak{m}_{C}(z)$ give rise to their local quotient rings

$$
\begin{gathered}
C_{\mathfrak{m}_{C}(z)} \subseteq B_{\mathfrak{m}_{B}(z)} \subseteq A_{\mathfrak{m}_{A}(z)} \text { with maximal ideals } \\
C_{\mathfrak{m}_{C}(z)} \mathfrak{m}_{C}(z)=\sum_{i=1}^{n} C_{\mathfrak{m}_{C}(z)}\left(s-z_{i}\right) \subseteq B_{\mathfrak{m}_{B}(z)} \mathfrak{m}_{B}(z) \subseteq \\
\subseteq A_{\mathfrak{m}_{A}(z)} \mathfrak{m}_{A}(z)=\sum_{i=1}^{n} A_{\mathfrak{m}_{A}(z)}\left(s-z_{i}\right) .
\end{gathered}
$$

Recall from Lemma 6.8 that $A_{\mathfrak{m}_{A}(z)}$ is noetherian.

Theorem 7.3. Assume a ring $B$ with

1. $\mathbb{C}[s] \subseteq B \subseteq A=\mathcal{O}\left(\mathbb{C}_{s}^{n}\right)$.

2. For each $z \in \mathbb{C}^{n}: \mathfrak{m}_{B}(z):=B \bigcap \mathfrak{m}_{A}(z)=\sum_{i=1}^{n} B\left(s_{i}-z_{i}\right)$.

3. For each $z \in \mathbb{C}^{n}$ the quotient ring $B_{\mathfrak{m}_{B}(z)}$ is noetherian.

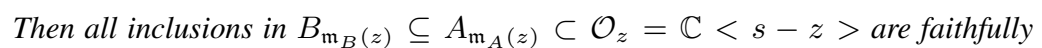
flat.

Proof. Since $B_{\mathfrak{m}_{B}(z)} \mathfrak{m}_{B}(z) \subseteq A_{\mathfrak{m}_{A}(z)} \mathfrak{m}_{A}(z)$ it suffices to show that these extensions are flat [4, Porp. I.3.9,(e)]. If $N$ is a noetherian local ring with maximal ideal $\mathfrak{n}$ we let $\widehat{N}:=\lim _{k} N / \mathfrak{n}^{k} \supseteq N$ denote its completion. The extension $N \subseteq \widehat{N}$ is then f.f. [4, Prop. III.3.9, III.3.11].

For $\mu \in \mathbb{N}^{n}$ we introduce $|\mu|:=\mu_{1}+\cdots+\mu_{n}$. The equations $B / \mathfrak{m}_{B}(z)=\mathbb{C}$ and $\mathfrak{m}_{B}(z)=\sum_{i=1}^{n} B\left(s_{i}-z_{i}\right)$ imply by induction that for $k>0$ one has

$$
\begin{gathered}
\mathfrak{m}_{B}(z)^{k}=\sum_{\mu \in \mathbb{N}^{n},|\mu|=k} B(s-z)^{\mu} \text { and } \\
B / \mathfrak{m}_{B}(z)^{k}=\sum_{|\mu|<k} \mathbb{C} \frac{(s-z)^{\mu}}{(s-z)^{\mu}}:=(s-z)^{\mu}+\mathfrak{m}_{B}(z)^{k},
\end{gathered}
$$

The same calculations for $C$ and $A$ instead of $B$ furnish

$$
\begin{aligned}
C / \mathfrak{m}_{C}(z)^{k} & =\oplus_{|\mu|<k} \mathbb{C}\left((s-z)^{\mu}+\mathfrak{m}_{B}(z)^{k}\right), \\
A / \mathfrak{m}_{A}(z)^{k} & =\oplus_{|\mu|<k} \mathbb{C}\left((s-z)^{\mu}+\mathfrak{m}_{A}(z)^{k}\right)
\end{aligned}
$$

where it is well-known that the $\mathbb{C}$-generators $\overline{(s-z)^{\mu}}$ are $\mathbb{C}$-linearly independent Moreover there are the canonical maps

$$
\begin{gathered}
C / \mathfrak{m}_{C}(z)^{k} \rightarrow B / \mathfrak{m}_{B}(z)^{k} \rightarrow A / \mathfrak{m}_{A}(z)^{k}, \\
(s-z)^{\mu}+\mathfrak{m}_{C}(z)^{k} \mapsto(s-z)^{\mu}+\mathfrak{m}_{B}(z)^{k} \mapsto(s-z)^{\mu}+\mathfrak{m}_{A}(z)^{k} .
\end{gathered}
$$

Due to the canonical bases in (91) all maps in (92) are indeed isomorphisms. Moreover there are the canonical isomorphisms

$$
B / \mathfrak{m}_{B}(z)^{k} \cong B_{\mathfrak{m}_{B}(z)} /\left(B_{\mathfrak{m}_{B}(z)} \mathfrak{m}_{B}(z)\right)^{k}
$$

and likewise for $C$ and $A$. From the isomorphisms in (92) we thus infer the isomorphisms

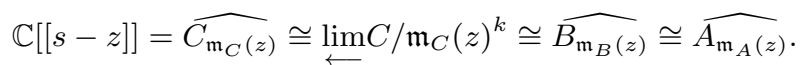


In the same fashion one has $A_{\mathfrak{m}_{A}(z)} \subset \mathcal{O}_{z}$ and proves the isomorphism $\widehat{A_{\mathfrak{m}_{A}(z)}} \cong$ $\widehat{\mathcal{O}_{z}}$. Summing up we obtain

$$
\begin{aligned}
& B_{\mathfrak{m}_{B}(z)} \subseteq A_{\left.\mathfrak{m}_{(} z\right)} \\
& \bigcap_{B_{\mathfrak{m}_{B}(z)}} \cong \frac{\mathcal{O}_{z}}{A_{\mathfrak{m}_{(}(z)}} \cong \widehat{\mathcal{O}_{z}} \cong \mathbb{C}[[s-z]]
\end{aligned}
$$

where all vertical inclusions are of the form $N \subseteq \widehat{N}$ for a noetherian local ring $N$ from above and hence f.f. and where we identify all rings in the second row for notational simplicity. Since

$$
\begin{gathered}
\widehat{B_{\mathfrak{m}_{B}(z)}} \otimes_{B_{\mathfrak{m}_{B}(z)}} M=\widehat{B_{\mathfrak{m}_{B}(z)}} \otimes_{\mathcal{O}_{z}}\left(\mathcal{O}_{z} \otimes_{B_{\mathfrak{m}_{B}(z)}} M\right), M \in_{B_{\mathfrak{m}_{B}(z)}} \text { Mod, } \\
\text { and } \widehat{B_{\mathfrak{m}_{B}(z)}} \otimes_{B_{\mathfrak{m}_{B}(z)}}(-) \text { and } \widehat{\mathcal{O}_{z}} \otimes_{\mathcal{O}_{z}}(-) \text { with } \widehat{B_{\mathfrak{m}_{B}(z)}}=\widehat{\mathcal{O}_{z}},
\end{gathered}
$$

are faithfully exact so is the functor $\mathcal{O}_{z} \otimes_{B_{\mathfrak{m}_{B}(z)}}(-)$ which signifies that the

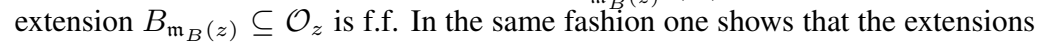
$B_{\mathfrak{m}_{B}(z)} \subseteq A_{\mathfrak{m}_{A}(z)}$ and $A_{\mathfrak{m}_{A}(z)} \subseteq \mathcal{O}_{z}$ are f.f.

The next theorem treats the elimination problem for ${ }_{B} W$-behaviors. Consider

$$
\begin{gathered}
V_{i} \subseteq B^{1 \times q_{i}}, i=1,2,3, P_{1} \in B^{q_{1} \times q_{2}}, P_{2} \in B^{q_{2} \times q_{3}}, \text { with } \\
V_{1} P_{1} \subseteq V_{2}, V_{2} P_{2} \subseteq V_{3}, \mathcal{B}_{i}:=V_{i}^{\perp} \subseteq W^{q_{i}}, i=1,2,3, \\
M_{1}:=B^{1 \times q_{1}} / V_{1} \stackrel{\left(\circ P_{1}\right)_{\text {ind }}}{\longrightarrow} M_{2}:=B^{1 \times q_{2}} / V_{2} \stackrel{\left(\circ P_{2}\right)_{\text {ind }}}{\longrightarrow} M_{3}:=B^{1 \times q_{3}} / V_{3} \\
\mathcal{B}_{1} \stackrel{P_{1} \circ}{\longleftarrow} \mathcal{B}_{2} \stackrel{P_{2} \circ}{\longleftarrow} \mathcal{B}_{3} .
\end{gathered}
$$

Notice that the $V_{i}$ are arbitrary, not necessarily closed submodules of $B^{1 \times q_{i}}$. The preceding module sequence gives rise to the localized resp. tensorized sequences

$$
\begin{aligned}
\left(M_{1}\right)_{\mathfrak{m}_{B}(z)}:= & B_{\mathfrak{m}_{B}(z)}^{1 \times q_{1}} / B_{\mathfrak{m}_{B}(z)} V_{1} \stackrel{\left(\circ P_{1}\right)_{\text {ind }}}{\longrightarrow}\left(M_{2}\right)_{\mathfrak{m}_{B}(z)}:=B_{\mathfrak{m}_{B}(z)}^{1 \times q_{2}} / B_{\mathfrak{m}_{B}(z)} V_{2} \longrightarrow \\
& \stackrel{\left(\circ P_{2}\right)_{\text {ind }}}{\longrightarrow}\left(M_{3}\right)_{\mathfrak{m}_{B}(z)}:=B_{\mathfrak{m}_{B}(z)}^{1 \times q_{3}} / B_{\mathfrak{m}_{B}(z)} V_{3}, z \in \mathbb{C}^{n},
\end{aligned}
$$

resp.

$$
\begin{gathered}
\mathcal{O}_{z} \otimes_{B} M_{1}=\mathcal{O}_{z} \otimes_{B_{\mathfrak{m}_{B}(z)}}\left(M_{1}\right)_{\mathfrak{m}_{B}(z)}=\mathcal{O}_{z}^{1 \times q_{1}} / \mathcal{O}_{z} V_{1} \stackrel{\left(\circ P_{1}\right)_{\text {ind }}}{\longrightarrow} \\
\mathcal{O}_{z} \otimes_{B} M_{2}=\mathcal{O}_{z} \otimes_{B_{\mathfrak{m}_{B}(z)}}\left(M_{2}\right)_{\mathfrak{m}_{B}(z)}=\mathcal{O}_{z}^{1 \times q_{2}} / \mathcal{O}_{z} V_{2} \stackrel{\left(\circ P_{2}\right)_{\text {ind }}}{\longrightarrow} \\
\mathcal{O}_{z} \otimes_{B} M_{3}=\mathcal{O}_{z} \otimes_{B_{\mathfrak{m}_{B}(z)}}\left(M_{3}\right)_{\mathfrak{m}_{B}(z)}=\mathcal{O}_{z}^{1 \times q_{3}} / \mathcal{O}_{z} V_{3}, z \in \mathbb{C}^{n} .
\end{gathered}
$$

Theorem 7.4. (Elimination for generalized ${ }_{B} W$-behaviors) Assume that the ring $B$ has the three properties of Theorem 7.3. Data from (95). Then

$$
\mathcal{B}_{1} \stackrel{P_{1} \circ}{\longleftarrow} \mathcal{B}_{2} \stackrel{P_{2} \circ}{\longleftarrow} \mathcal{B}_{3}
$$

is exact if and only if all sequences (96) are exact. This is the case if the original sequence

$$
M_{1} \stackrel{\left(\circ P_{1}\right)_{\text {ind }}}{\longrightarrow} M_{2} \stackrel{\left(\circ P_{2}\right)_{\text {ind }}}{\longrightarrow} M_{3}
$$

is exact. For $V_{1}:=\left(\circ P_{1}\right)^{-1}\left(V_{2}\right)$ this implies the elimination equality $P_{1} \circ V_{2}^{\perp}=$ $V_{1}^{\perp}$ whereas for $V_{2}:=0$ one gets $P_{1} \circ W^{q_{2}}=\operatorname{ker}\left(\circ P_{1}\right)^{\perp}$, i.e., the fundamental principle. In particular, the module ${ }_{B} W$ satifies the weak Baer condition of Lemma 2.4 and is divisible. If $B$ is coherent then the image of $a_{B} W$-behavior is again such. 
Proof. We use Result 6.1, Th. 6.2 and the f.f. extensions $B_{\mathfrak{m}_{B}(z)} \subset \mathcal{O}_{z}$ of Th. 7.3. Consider

$$
V_{i} \subseteq A V_{i} \subseteq U_{i}:=\operatorname{cl}_{A}\left(A V_{i}\right) \subseteq A^{1 \times q_{i}} \text {, hence } \mathcal{B}_{i}=V_{i}^{\perp}=U_{i}^{\perp} .
$$

We have $\left(M_{i}\right)_{\mathfrak{m}_{B}(z)}=B_{\mathfrak{m}_{B}(z)}^{1 \times q_{i}} / B_{\mathfrak{m}_{B}(z)} V_{i}$. From Result 6.1, item 8, we infer that

$$
\begin{gathered}
\left(U_{i}\right)_{z}=\mathcal{O}_{z}\left(A V_{i}\right)=\mathcal{O}_{z} V_{i} \subseteq \mathcal{O}_{z}^{1 \times q_{i}} \text { and thus } \\
\left(A^{1 \times q_{i}} / U_{i}\right)_{z}=\mathcal{O}_{z}^{1 \times q_{i}} /\left(U_{i}\right)_{z}=\mathcal{O}_{z}^{1 \times q_{i}} / \mathcal{O}_{z} V_{i}=\mathcal{O}_{z} \otimes_{B_{\mathfrak{m}_{B}(z)}}\left(M_{i}\right)_{\mathfrak{m}_{B}(z)}
\end{gathered}
$$

Hence

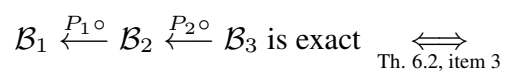

$$
\begin{aligned}
& A^{1 \times q_{1}} / U_{1} \stackrel{\left(\circ P_{1}\right)_{\text {ind }}}{\longrightarrow} A^{1 \times q_{2}} / U_{2} \stackrel{\left(\circ P_{2}\right)_{\text {ind }}}{\longrightarrow} A^{1 \times q_{3}} / U_{3} \text { is exact } \underset{\text { Result 6.1, item } 7}{\Longleftrightarrow} \\
& \forall z \in \mathbb{C}^{n}:\left(A^{1 \times q_{1}} / U_{1}\right)_{z}=\mathcal{O}_{z}^{1 \times q_{1}} / \mathcal{O}_{z} V_{1} \stackrel{\left(\circ P_{1}\right)_{\text {ind }}}{\longrightarrow}
\end{aligned}
$$

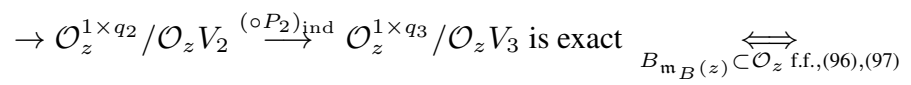

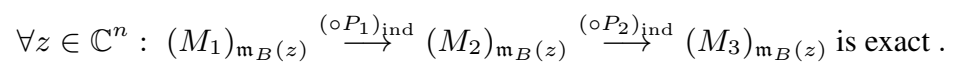

Corollary 7.5. Assume that B from (82) is coherent. Then the following properties are equivalent:

1. The ring B satisfies the assumptions of Th. 7.3 and thus of Th. 7.4 and has the equivalent properties of Cor. 7.2,(2).

2. The extension $B \subseteq A$ is faithfully flat.

Proof. 1. $\Longrightarrow 2 .: B \subseteq A$ is flat: It suffices to show that for a f.g. ideal $\mathfrak{b} \subseteq B$ the map $A \otimes_{B}$ inj : $A \otimes_{B} \mathfrak{b} \rightarrow A \otimes_{B} B=A$ is injective [4, Prop. I.2.1, Rem. on p.13]. Since $B$ is coherent so is $\mathfrak{b}$. Therefore we show more generally that a short exact sequence

$$
M_{1}:=0 \rightarrow M_{2}=B^{1 \times q_{2}} / V_{2} \stackrel{f:=\left({ }^{\circ P_{2}}\right)_{\text {ind }}}{\longrightarrow} M_{3}=B^{1 \times q_{3}} / V_{3}, V_{i} \text { f.g. in } B^{1 \times q_{i}},
$$

of coherent or f.p. $B$-modules induces a monomorphism

$$
A \otimes_{B} f: A \otimes_{B} M_{2}=A^{1 \times q_{2}} / A V_{2} \rightarrow A \otimes_{B} M_{3}=A^{1 \times q_{3}} / A V_{3} .
$$

Here we used the notations from Th. 7.4 and its proof with $M_{1}=0$. Since $V_{i}$ is f.g. the modules $A V_{i} \subseteq A^{1 \times q_{i}}$ are f.g. too and thus closed, i.e., $A V_{i}=$ $\operatorname{cl}_{A}\left(A V_{i}\right)=: U_{i}$. The exactness of (99) then follows from the exact sequences in (98). That $B \subseteq A$ is even f.f. is a consequence of Cor. 7.2,(2).

2. $\Longrightarrow 1 .:$ (a) For $z \in \mathbb{C}^{n}$ consider the ideal $\mathfrak{b}:=\sum_{i=1}^{n} B\left(s_{i}-z_{i}\right) \subseteq \mathfrak{m}_{B}(z)$. Since $B \subseteq A$ is f.f. we infer [4, Prop. I.3.9]

$\sum_{i=1}^{n} B\left(s_{i}-z_{i}\right)=\mathfrak{b}=B \bigcap A \mathfrak{b}=B \bigcap \sum_{i=1}^{n} A\left(s_{i}-z_{i}\right)=B \bigcap \mathfrak{m}_{A}(z)=\mathfrak{m}_{B}(z)$.

This is condition 2. of Th. 7.3.

(b) Since $B \subseteq A$ and $A \subseteq A_{\mathfrak{m}_{A}(z)}, z \in \mathbb{C}^{n}$, are flat so are

$$
B \subseteq A_{\mathfrak{m}_{A}(z)} \text { and } B_{\mathfrak{m}_{B}(z)} \subseteq\left(A_{\mathfrak{m}_{A}(z)}\right)_{\mathfrak{m}_{B}(z)}=A_{\mathfrak{m}_{A}(z)} .
$$

As an extension of local rings the latter inclusion is even f.f. Since $A_{\mathfrak{m}_{A}(z)}$ is noetherian by Lemma 6.8 so is $B_{\mathfrak{m}_{B}(z)}$ [4, Prop. I.3.11]. This is condition 3. of Th. 7.3. The remaining assertion follows from Cor. 7.2,(2). 
Corollary 7.6. $\left[1\right.$, Th. 2] The extension $C=\mathbb{C}[s] \subset A=\mathcal{O}\left(\mathbb{C}_{s}^{n}\right)$ is faithfully flat.

Proof. We use the notations of Th. 7.4 and its proof. Consider the case $B:=C$. The ring $C$ is noetherian and the $\mathfrak{m}_{C}(z)$ are all maximal ideals of $C$ by Hilbert's Nullstellensatz. Therefore the exactness of $M_{1} \rightarrow M_{2} \rightarrow M_{3}$ is equivalent with the exactness of the sequences (96) [4, Th. II.3.1]. Moreover $V_{i}$ is f.g., hence $A V_{i}$ is f.g. and closed, thus

$$
U_{i}=A V_{i} \text { and } A \otimes_{B} M_{i}=A \otimes_{B}\left(B^{1 \times q_{i}} / V_{i}\right)=A^{1 \times q_{i}} / A V_{i}=A^{1 \times q_{i}} / U_{i} .
$$

Thus the exactness of $M_{1} \rightarrow M_{2} \rightarrow M_{3}$ is equivalent with that of the sequence $A \otimes_{B} M_{1} \rightarrow A \otimes_{B} M_{2} \rightarrow A \otimes_{B} M_{3}$. But this signifies that $C \subset A$ is f.f.

We finally show that the three properties of Theorem 7.3 are satisfied in dimension one and especially for the case of DD-equations with incommensurate delays, i.e., for

$$
\begin{gathered}
\mathbb{C}[s] \subseteq B=\operatorname{quot}(B) \bigcap A \subseteq A:=\mathcal{O}\left(\mathbb{C}_{s}\right) \text { and especially for } \\
B:=\operatorname{quot}\left(\mathbb{C}\left[s, \sigma, \sigma^{-1}\right]\right) \bigcap A=\mathbb{C}(s)\left[\sigma, \sigma^{-1}\right] \bigcap A, n=1, m>1
\end{gathered}
$$

with the data from (83) and (85). In the ring $A=\mathcal{O}\left(\mathbb{C}_{s}\right)$ the closed and especially the f.g. ideals are principal. The closed maximal ideals of $A$ are the

$\mathfrak{m}_{A}(z)=A(s-z)=\{f \in A ; f(z)=0\}, z \in \mathbb{C}$. The local rings $A_{\mathfrak{m}_{A}(z)} \subset \mathcal{O}_{z}$

are discrete valuation rings, i.e., principal ideal domains with the unique prime $s-z$ up to association. For each $z \in \mathbb{C}$ and each nonzero $f \in A$ there is a unique representation

$$
f=g(s-z)^{k}, g \in A \backslash \mathfrak{m}_{A}(z),
$$

where $k$ is the order of $f$ at $z$.

Theorem 7.7. (Elimination in the one-dimensional case) Any ring $B$ as in (100) satisfies the assumptions of Th. 7.3 and thus of Th. 7.4, in particular images of generalized behaviors are again such.

This solves, but only for analytic behaviors, the open problem in [13, behind Th. 3.14 ] and seems to be the first elimination result for DD-behaviors with incommensurate delays. We do presently not know whether $B=\operatorname{quot}\left(\mathbb{C}\left[s, \sigma, \sigma^{-1}\right]\right) \cap A$ is coherent and whether $B \subseteq A$ is faithfully flat, i.e., whether the equivalent properties of Cor. 7.5 hold. For $m=1$ this ring is a Bézout domain [12] and thus coherent and $B \subset A$ is f.f.

Proof. 1. We prove that $\mathfrak{m}_{B}(z):=B \bigcap \mathfrak{m}_{A}(z)=B(s-z)$ : Obviously $s-z$ is contained in $\mathfrak{m}_{B}(z)$. Now assume $0 \neq f \in B$ and $f(z)=0$. By (102) there is a unique representation

$$
\begin{gathered}
f=g(s-z)^{k}, k>0 \text { since } f(z)=0, g \in A \backslash \mathfrak{m}_{A}(z) \Longrightarrow g=\frac{f}{(s-z)^{k}} \in \\
q u o t(B) \bigcap A=B \Longrightarrow f \in B(s-z) \Longrightarrow \mathfrak{m}_{B}(z)=B(s-z) .
\end{gathered}
$$

2. We show that $B_{\mathfrak{m}_{B}(z)}$ is also a discrete valuation domain with prime element $s-z$ and hence especially noetherian: Let

$$
\begin{gathered}
f=\frac{b_{1}}{b_{2}} \in B_{\mathfrak{m}_{B}(z)}, b_{2} \in B \backslash \mathfrak{m}_{B}(z) \underset{(103)}{\Longrightarrow} b_{1}=b_{3}(s-z)^{k} \text { with } b_{3} \in B \backslash \mathfrak{m}_{B}(z) \\
\Longrightarrow f=\frac{b_{3}}{b_{2}}(s-z)^{k}, \frac{b_{3}}{b_{2}} \text { unit in } B_{\mathfrak{m}_{B}(z)} .
\end{gathered}
$$


Hence each $f \in B_{\mathfrak{m}_{B}(z)}$ has a unique representation $f=u(s-z)^{k}$ with a unit $u \in B_{\mathfrak{m}_{B}(z)}$. This signifies that $B_{\mathfrak{m}_{B}(z)}$ is a discrete valuation domain.

We notice that the faithful flatness of $B_{\mathfrak{m}_{B}(z)} \subset \mathcal{O}_{z}$ according to Th. 7.3 is obvious in the situation of the preceding theorem since every torsionfree module like $\mathcal{O}_{z}$ over any principal ideal domain like $B_{\mathfrak{m}_{B}(z)}$ is flat.

\section{Characteristic variety and controllability}

Let $\left(Z, \mathcal{O}_{Z}\right)$ be a Stein space and $A:=\mathcal{O}_{Z}(Z)$ its Stein algebra as in Section 6. If $M$ and $N$ are Stein modules over $A$ then so is $\operatorname{Hom}_{A}(M, N)$ and the canonical maps

$$
\begin{aligned}
& \operatorname{Hom}_{A}(M, N)_{z}=\mathcal{O}_{z} \otimes_{A} \operatorname{Hom}_{A}(M, N) \longrightarrow \operatorname{Hom}_{\mathcal{O}_{z}}\left(M_{z}, N_{z}\right), \\
& \phi_{z}=1 \otimes \phi \quad \mapsto \quad \operatorname{id}_{\mathcal{O}_{z}} \otimes \phi
\end{aligned}
$$

are isomorphisms [9, p.398]. Therefore we identify $\phi_{z}=\operatorname{id}_{\mathcal{O}_{z}} \otimes \phi$. As usual we define the dual $M^{*}$ and double dual $M^{* *}$ of a module ${ }_{A} M$ and the canonical Gelfand homomorphism

$$
\begin{gathered}
M^{*}:=\operatorname{Hom}_{A}(M, A) \text { and } \rho_{M}: M \rightarrow M^{* *}, x \mapsto \rho_{M}(x), \text { with } \\
\rho_{M}(x)(\varphi):=\varphi(x), \varphi \in M^{*} . \text { Let } T(M):=\operatorname{ker}\left(\rho_{M}\right) . \text { Then } T(M)_{z}= \\
T\left(M_{z}\right):=\operatorname{ker}\left(\rho_{M_{z}}: M_{z} \rightarrow M_{z}^{* *}=\operatorname{Hom}_{\mathcal{O}_{z}}\left(\operatorname{Hom}_{\mathcal{O}_{z}}\left(M_{z}, \mathcal{O}_{z}\right), \mathcal{O}_{z}\right)\right)
\end{gathered}
$$

where the last equality comes from Th. 6.1, item 7., and (104).

The support of a Stein module $M$ is defined as $\operatorname{supp}(M):=\left\{z \in Z ; M_{z} \neq 0\right\}$. This is an analytic subset of $Z[15, \S 4.1 .1]$. We define the finite-dimensional $\mathbb{C}$ space

$$
\begin{gathered}
M(z):=A / \mathfrak{m}(z) \otimes_{A} M=M / \mathfrak{m}(z) M=\mathcal{O}_{z} / \mathfrak{m}_{z} \otimes_{\mathcal{O}_{z}} M_{z}=M_{z} / \mathfrak{m}_{z} M_{z} \text { with } \\
A / \mathfrak{m}(z)=\mathcal{O}_{z} / \mathfrak{m}_{z}=\mathbb{C}, f+\mathfrak{m}(z)=f_{z}+\mathfrak{m}_{z}=f(z) .
\end{gathered}
$$

If

$$
\begin{gathered}
M=A^{1 \times q} / A^{1 \times k} R, R \in A^{k \times q}, \text { is f.p. then } \\
M(z)=\mathbb{C}^{1 \times q} / \mathbb{C}^{1 \times k} R(z) \text { and } \operatorname{dim}_{\mathbb{C}}(M(z))=q-\operatorname{rank}(R(z)) .
\end{gathered}
$$

Recall that a f.g. module over the local algebra $\mathcal{O}_{z}$ is free if and only if it is projective. The characteristic variety of a Stein module is defined as

$$
\operatorname{char}(M):=\left\{z \in Z ; M_{z} \text { is not free }\right\} .
$$

The Stein space is called reduced if all local rings $\mathcal{O}_{z}, z \in Z$, have no nilpotent elements. A Stein manifold and especially $\mathbb{C}^{n}$ are reduced.

Result 8.1. Let $\left(Z, \mathcal{O}_{Z}\right)$ denote a finite-dimensional Stein space with a countable basis of the topology and its Stein algebra $A:=\mathcal{O}_{Z}(Z)$ of global sections and let $M$ be a Stein module.

1. [15, Th. in \$4.4.1] The characteristic variety is an analytic subset of $Z$. If $Z$ is reduced, for instance $Z=\mathbb{C}^{n}$, then $\operatorname{char}(M)$ is nowhere dense, especially $\operatorname{char}(M) \subsetneq Z$.

2. [15, Crit. 2 in $\$ 4.4 .1]$ If $Z$ is reduced then

$Z \backslash \operatorname{char}(M)=\left\{z \in Z ; \operatorname{dim}_{\mathbb{C}} M(z)\right.$ is constant in a neighborhood of $\left.z\right\}$ 
3. [9, Satz 6.3] The Stein module $M$ is projective if and only if all $M_{z}, z \in Z$, are free, i.e., if $\operatorname{char}(M)=\emptyset$.

4. [9, p.402] If $Z$ is contractible then every projective Stein module is free. This applies to $Z=\mathbb{C}^{n}$ in particular.

Assumption 8.2. In the sequel of this section we assume that $Z$ is locally irreducible, i.e., that all $\mathcal{O}_{z}, z \in Z$, are integral domains [15, §1.1.5], and that also $A=\mathcal{O}_{Z}(Z)$ is an integral domain. This is the case for a connected Stein manifold and especially for $\mathbb{C}^{n}[15, \S 9.1 .1]$. Then the identity theorem holds in $Z$ (Lemma 6.8) and items 1 and 2. of Result 8.1 are applicable. Let quot $(A)$ denote the quotient field of a domain $A$. We infer the inclusions

$$
A \subset \mathcal{O}_{z} \subset \operatorname{quot}\left(\mathcal{O}_{z}\right) \supset \operatorname{quot}(A) \supset A .
$$

For a Stein $A$-module $M$ and $M_{z}=\mathcal{O}_{z} \otimes_{A} M$ this implies

$$
\begin{gathered}
\operatorname{quot}\left(\mathcal{O}_{z}\right) \otimes_{\mathcal{O}_{z}} M_{z}=\operatorname{quot}\left(\mathcal{O}_{z}\right) \otimes_{\text {quot }(A)}\left(\operatorname{quot}(A) \otimes_{A} M\right) \text { and hence } \\
\operatorname{dim}_{\text {quot }(A)}\left(\operatorname{quot}(A) \otimes_{A} M\right)=\operatorname{dim}_{\text {quot }\left(\mathcal{O}_{z}\right)}\left(\operatorname{quot}\left(\mathcal{O}_{z}\right) \otimes_{\mathcal{O}_{z}} M_{z}\right) .
\end{gathered}
$$

We define the ranks of $M$ and $M_{z}$ by

$$
\begin{aligned}
\operatorname{rank}(M):= & \operatorname{dim}_{\mathrm{quot}(A)}\left(\mathrm{quot}(A) \otimes_{A} M\right) \text { and likewise } \operatorname{rank}\left(M_{z}\right), \\
& \text { hence } \operatorname{rank}(M)=\operatorname{rank}\left(M_{z}\right) \text { for all } z \in Z .
\end{aligned}
$$

If

$$
\begin{aligned}
& M=A^{1 \times q} / A^{1 \times k} R, R \in A^{k \times q}, \text { then with } K:=\operatorname{quot}(A): \\
& K \otimes_{A} M=K^{1 \times q} / K^{1 \times k} R \text { and } \operatorname{rank}(M)=q-\operatorname{rank}(R) .
\end{aligned}
$$

The Lemma of Krull/Nakayama implies that the dimension $\operatorname{dim}_{\mathbb{C}}\left(\mathcal{O}_{z} / \mathfrak{m}_{z} \otimes_{\mathcal{O}_{z}} M_{z}\right)=$ $\operatorname{dim}_{\mathbb{C}} M(z)$ is the minimal number of generators of $M_{z}$, hence

$$
\operatorname{rank}(M)=\operatorname{rank}\left(M_{z}\right) \leq \operatorname{dim}_{\mathbb{C}} M(z) .
$$

Moreover $M_{z}$ is free if and only if $\operatorname{rank}\left(M_{z}\right)=\operatorname{dim}_{\mathbb{C}} M(z)$. Thus

$$
\begin{gathered}
\operatorname{char}(M)=\left\{z \in Z ; \operatorname{rank}(M)<\operatorname{dim}_{\mathbb{C}}(M(z))\right\} \text { and } \\
\operatorname{char}(M)=\{z \in Z ; \operatorname{rank}(R(z))<\operatorname{rank}(R)\} \text { if } M=A^{1 \times q} / A^{1 \times k} R .
\end{gathered}
$$

The torsion module of any $A$-module $M$ is defined by

$$
\operatorname{tor}\left({ }_{A} M\right):=\{x \in M ; \exists 0 \neq f \in A \text { with } f x=0\} .
$$

For f.g. $M$ it is known and easily shown that

$$
\operatorname{tor}(M)=T(M):=\operatorname{ker}\left(\rho_{M}: M \rightarrow M^{* *}=\operatorname{Hom}_{A}\left(\operatorname{Hom}_{A}(M, A), A\right)\right)
$$

where the Gelfand map $\rho_{M}$ is defined in (105). For a Stein module $M$ this implies

$$
T(M)_{z}=\operatorname{tor}\left(\mathcal{O}_{z} M_{z}\right) \text { for } z \in Z \text { and } T(M)=\operatorname{tor}\left({ }_{A} M\right) \text { if } M \in_{A} \operatorname{Mod}^{S t, f} \text {. }
$$

A free module is torsionfree, hence for all Stein modules $M$

$$
\begin{gathered}
\operatorname{supp}(T(M))=\left\{z \in Z ; M_{z} \text { is not torsionfree }\right\} \\
\subseteq \operatorname{char}(M):=\left\{z \in Z ; M_{z} \text { is not free }\right\} .
\end{gathered}
$$

For $M \in{ }_{A} \operatorname{Mod}^{S t, f}: T(M)=\operatorname{tor}(M), \operatorname{supp}(T(M))=\operatorname{supp}(\operatorname{tor}(M))$, especially $M$ is torsionfree $\Longleftrightarrow \operatorname{tor}(M)=0 \Longleftrightarrow \operatorname{supp}(\operatorname{tor}(M))=\emptyset$.

The following Theorem and Definition is just a reformulation of the preceding statements. 
Theorem and Definition 8.3. (Controllability) Let A be a Stein algebra satisfying Assumption 8.2 and let $W$ be the signal module $A^{\prime}$ or an isomorphic one, for instance $A=\mathcal{O}\left(\mathbb{C}_{s}^{n}\right)$ and $W=\mathcal{O}\left(\mathbb{C}_{x}^{n} ; \exp \right) \cong \mathcal{O}\left(\mathbb{C}_{s}^{n}\right)^{\prime}$. Let $U \subseteq A^{1 \times q}$ be closed, for instance f.g., with its generalized behavior ${ }_{A} W$-behavior $\mathcal{B}:=U^{\perp}$, thus $U=\mathcal{B}^{\perp}$, and module $M:=A^{1 \times q} / U \in{ }_{A} \operatorname{Mod}^{S t, f}$.

1. The following statements are equivalent:

(a) The behavior $\mathcal{B}$ is controllable, i.e., by definition, its module $M$ is torsionfree or, equivalently, all modules $M_{z}, z \in Z$, are torsionfree.

(b) $\mathcal{B}$ admits an image representation, i.e., there are $r \in \mathbb{N}$ and a matrix $P \in A^{q \times r}$ such that $P \circ W^{r}=\mathcal{B}$

(c) $\operatorname{supp}\left(\operatorname{tor}\left({ }_{A} M\right)\right)=\emptyset$.

2. The following statements are equivalent:

(a) $\mathcal{B}$ is spectrally controllable i.e., by definition, its characteristic variety $\operatorname{char}(\mathcal{B}):=\operatorname{char}(M)$ is empty.

(b) The module $M$ is projective.

3. If $Z$ is contractible, for instance $Z=\mathbb{C}^{n}$ and $A=\mathcal{O}\left(\mathbb{C}_{s}^{n}\right)$, then the following statements are equivalent:

(a) $\mathcal{B}$ is spectrally controllable.

(b) $M$ is free.

(c) $\mathcal{B}$ admits an injective image representation $P \circ: W^{r} \cong \mathcal{B}$.

A spectrally controllable generalized behavior is, of course, controllable.

4. For the one-dimensional Stein algebra $\mathcal{O}\left(\mathbb{C}_{s}\right)$ and signal module $\mathcal{O}\left(\mathbb{C}_{x} ; \exp \right)$ generalized behaviors coincide with behaviors and controllability with spectral controllability. In particular, $\mathcal{O}\left(\mathbb{C}_{s}\right)$ is a Bézout and a Prüfer domain [4, Ex. VII.1.20, Ex. VII.2.12].

Proof. 1 . Only the equivalence $(a) \Longleftrightarrow(b)$ has to be shown. But the f.g. module $M$ is torsionfree if and only if it admits an embedding into some f.g. free module. Thus

$$
M \text { is torsionfree } \Longleftrightarrow
$$

There is a monomorphism $(\circ P)_{\text {ind }}: M=A^{1 \times q} / U \rightarrow A^{1 \times r}=A^{1 \times r} / 0$

$$
\text { Th. } \underset{6.2 \text {,item 3. }}{\Longleftrightarrow} \text { There is an epimorphism } P \circ: 0^{\perp}=W^{r} \rightarrow U^{\perp}=\mathcal{B} \text {. }
$$

3. The equivalence of 3.(b) and 3.(c) is shown as in the first part 1. of the proof. 4. Each local ring $\mathcal{O}_{z}=\mathbb{C}\langle s-z\rangle$ is a discrete valuation ring, i.e., a principal ideal domain with the unique prime element $s-z$, up to association. Any f.g. torsionfree $\mathcal{O}_{z}$-module is free. Hence f.g. torsionfree $\mathcal{O}\left(\mathbb{C}_{s}\right)$-modules are free. In particular, f.g. ideals are free and thus principal which, by definition, is the Bézout property.

\section{Concluding remarks}

In three standard cases of systems theoretic significance and various generalizations thereof we have shown that strong elimination and duality properties hold for commutative operator rings $A$ of convergent power series and associated signal modules $W$ of entire functions. The rings contain the partial derivatives and all translation operators so that linear systems and behaviors governed by partial differential-difference equations can be defined and studied. The prototypical cases are the following: 
1. In Th. 6.2 $A=\mathcal{O}\left(\mathbb{C}_{s}^{n}\right)$ is the ring of entire functions on $\mathbb{C}^{n}$ in variables $s=\left(s, \cdots, s_{n}\right)$ and $W=\mathcal{O}\left(\mathbb{C}_{x}^{n} ; \exp \right)$ is the space of entire functions in $x=\left(x_{1}, \cdots, x_{n}\right)$ of at most exponential growth with the action $\circ$ of $A$ on $W$ which extends $s_{i} \circ w=\partial w / \partial x_{i}$ and $\left(e^{y \bullet s} \circ w\right)(x)=w(x+y)$. The ring $A$ is a Stein algebra and $W$ is an injective cogenerator for the category of f.g. Stein modules, i.e., $\operatorname{Hom}_{A}(-, W)$ is faithfully exact on this category (compare Th. 6.2,(3)). This implies strong elimination and duality properties for ${ }_{A} W$-behaviors. As consequences thereof Th. 6.5 resp. Th. 7.7 prove elimination for periodic signals resp. delay-differential equations with incommensurate delays, Th. 7.7 solving an open problem of GlüsingLürssen/Vettori/Zampieri [13] for analytic signals. We also characterize controllable systems in Section 8.

2. $A=\mathbb{C}\langle s-z\rangle\left(\supset \mathcal{O}\left(\mathbb{C}_{s}^{n}\right)\right)$ is the ring of locally convergent power series near $z \in \mathbb{C}^{n}$ with the signal module $W_{z}=W_{0} e^{z \bullet x}$ where $W_{0} \subset W$ is the space of infra-exponential functions, i.e., of less than exponential growth.

3. $A=\mathcal{O}(K)\left(\supset \mathcal{O}\left(\mathbb{C}_{s}^{n}\right)\right)$ is the ring of holomorphic functions on a semianalytic and geometrically convex compact subset $K$ of $\mathbb{C}^{n}$, for instance on a compact polyhedron or polydisc, and the signal module $W_{K}$ is a subspace of $W$ of functions with explicitly given growth condition.

Th. 4.3 and 5.7 show that in cases (2) and (3) the ring $A$ is noetherian and the signal module is an injective cogenerator which gives rise to the strongest possible elimination and duality properties for the corresponding behaviors. The latter are interpreted as systems whose generalized frequencies of interest lie in the neighborhood of $z$ resp. the compact set $K$.

The following short remarks address questions of one of the referees:

4. We have not treated constructive and computational aspects of our theorems since such methods, let alone computer software, are widely lacking in the theory of several complex variables, in particular in all used and quoted books. In the simplest case of commensurate DD-equations Glüsing-Lürssen $[12, \S 3.6]$ discusses computability.

5. Non-commutative operator domains appear if one considers linear systems of partial differential-(difference) equations with variable coefficients on $\mathbb{R}^{n}$ or with constant coefficients on non-commutative Lie groups, compare [6] and the references given there. The mini-course [2] will also be of interest in this context. If $k$ is any field and $A$ any $k$-algebra (non-commutative, non-topological) the algebraic dual $A^{*}:=\operatorname{Hom}_{k}(A, k)$ is an injective $A$ cogenerator, compare (3), but in general $A^{*}$ is not an interesting function space. Serre [25] used non-commutative formal power series in the proof of the Campbell-Baker-Hausdorff formula for Lie algebras, Fliess [8] gave a systems theoretic application of such series. But to our knowledge deep analytic theorems concerning topological algebras of non-commuting power series with interesting topological duals, realized by the Laplace transform in our paper, have not yet been established.

\section{References}

[1] J. Apel, J. Stückrad, P. Tworzewski, T. Winiarski, Properties of Entire Functions over Polynomial Rings via Gröbner Bases, AAECC 14(2003), 1-10

[2] J.A. Ball, J.W. Helton, D. Kaliuzhnyi-Verbovetskyi, I. Klep, V. Vinnikov, Noncommutative multidimensional linear systems, analytic function theory 
and real algebraic geometry in the noncommutative setting, and overdetermined multidimensional linear systems: a minicourse, MTNS 2012, Melbourne

[3] C.A. Berenstein, M.A. Dostal, The Ritt theorem in several variables, Arkiv för matematik 12(1974), 267-280

[4] N. Bourbaki, Commutative Algebra, Hermann, Paris, 1972

[5] N. Bourbaki, Espaces Vectoriels Topologiques. Chapitres 1 à 5, Masson, Paris, 1981 (English translation: Topological Vector Spaces, Springer, New York, 1987)

[6] H. Bourlès, U. Oberst, Duality for Differential-Difference Systems over Lie Groups, SIAM J. Control Optim. 48(2009), 2051-2084

[7] H. Bourlès, U. Oberst, Elimination, fundamental principle and duality for analytic linear systems of partial differential-difference equations with constant coefficients, Proc. MTNS 2010, Budapest, 1551-1555

[8] M. Fliess, Réalisation locale des systèmes non-linéaires, algèbres de Lie filtrées transitives et séries génératrices non commutatives, Inv. Math. 71(1983), 521-537

[9] O. Forster, Zur Theorie der Steinschen Moduln und Algebren, Math.Z. 97(1967), 376-405

[10] J. Frisch, Points de platitude d'un morphisme d'espaces analytiques complexes, Inv. Math. 4(1967), 118-138

[11] H. Glüsing-Lürssen, A behavioral approach to delay-differential systems, SIAM J. Control Optim. 35(1997), 480-499

[12] H. Glüsing-Lürssen, Linear Delay-Differential Systems with Commensurate Delays: An Algebraic Approach, Lecture Notes in Mathematics 1770, Springer, Berlin, 2002

[13] H. Glüsing-Lürssen, P. Vettori, S. Zampieri, The algebraic structure of $D D$ systems: a behavioral perspective, Kybernetica 37(2001), 397-426

[14] H. Grauert, R. Remmert, Analytische Stellenalgebren, Springer, Heidelberg, 1971

[15] H. Grauert, R. Remmert, Coherent Analytic Sheaves, Springer, Berlin, 1984

[16] H. Grauert, R. Remmert, Theorie der Steinschen Räume, Springer, Heidelberg, 1977 (English translation: Theory of Stein Spaces, Springer, New York, 2004)

[17] R.C. Gunning, Introduction to Holomorphic Functions of Several Variables, Vol. III, Wadsworth and Brooks/Cole, Belmont, 1990

[18] L.C. Habets, System equivalence for AR-systems over rings-With an application to delay-differential systems, Math. Contr. Sign. Syst. 12(1999), 219-244

[19] L. Hörmander, An Introduction to Complex Analysis in Several Variables, Third Edition, North Holland, Amsterdam, 1991

[20] A. Martineau, Sur les fonctionelles analytiques et la transformation de Fourier-Borel, J. Ann. Math. (Jerusalem) 9(1963), 1-164

[21] M. Morimoto, An Introduction to Sato's Hyperfunctions, Transl. Math. Monographs 129, AMS, Providence, 1993

[22] H. Mounier, Algebraic interpretations of the spectral controllability of a linear delay system, Forum Math. 10(1998), 39-58

[23] D. Napp-Avelli, M. van der Put, S. Shankar, Periodic behaviors, to appear in SIAM J. Control Optim. 
[24] U. Oberst, Multidimensional Constant Linear Systems, Acta Appl. Math. 20(1990), 1-175

[25] J.P. Serre, Lie Algebra and Lie Groups, Benjamin, New York, 1965

[26] S. Shankar, The lattice structure of behaviors, SIAM J. Control Optim. 39(2001), 1817-1832

[27] S. Shankar, Geometric completeness of distributions spaces, Acta Appl. Math. 77(2003), 163-180

[28] S. Shankar, V.R. Sule, Algebraic geometric aspects of feedback stabilization, SIAM J. Control Optim. 30(1992), 11-30

[29] J.L. Taylor, Several Complex Variables with Connections to Algebraic Geometry and Lie Groups, American Mathematical Society, 2002

[30] P. Vettori, S. Zampieri, Some Results on Systems Described by Convolution Equations, IEEE Trans. Autom. Control 46(2001), 793-797 